\title{
COMPUTING COX RINGS
}

\author{
JÜRGEN HAUSEN, SIMON KEICHER AND ANTONIO LAFACE
}

\begin{abstract}
We consider modifications, for example blow ups, of Mori dream spaces and provide algorithms for investigating the effect on the Cox ring, for example verifying finite generation or computing an explicit presentation in terms of generators and relations. As a first application, we compute the Cox rings of all Gorenstein log del Pezzo surfaces of Picard number one. Moreover, we show computationally that all smooth rational surfaces of Picard number at most six are Mori dream surfaces and we provide explicit presentations of the Cox ring for those not admitting a torus action. Finally, we provide the Cox rings of projective spaces blown up at certain special point configurations.
\end{abstract}

\section{INTRODUCTION}

Generalizing the well known construction of the homogeneous coordinate ring of a toric variety [17, one associates to any normal complete variety $X$ defined over an algebraically closed field $\mathbb{K}$ of characteristic zero and having a finitely generated divisor class group $\mathrm{Cl}(X)$ its $C o x$ ring

$$
\mathcal{R}(X)=\bigoplus_{\mathrm{Cl}(X)} \Gamma\left(X, \mathcal{O}_{X}(D)\right) .
$$

A characteristic feature of the Cox ring is its divisibility theory: it allows unique factorization in the multiplicative monoid of homogeneous elements $3,9,26$. Projective varieties with finitely generated Cox ring are called Mori dream spaces [30]. Such a Mori dream space is determined by its Cox ring up to a finite choice of possible Mori chambers, which in turn correspond to GIT quotients of the action of $H=\operatorname{Spec} \mathbb{K}[\mathrm{Cl}(X)]$ on the total coordinate space $\operatorname{Spec} \mathcal{R}(X)$. In the surface case, the Cox ring $\mathcal{R}(X)$ even completely encodes $X$. Once the Cox ring of a variety $X$ is known in terms of generators and relations, this opens an approach to the explicit study of $X$. For example, in [12, Manin's conjecture was proven for the $E_{6}$-singular surface using such a presentation for its Cox ring. Explicit Cox ring computations are often based on a detailed knowledge of the geometry of the underlying variety; the pioneer work in this direction concerns (generalized) smooth del Pezzo surfaces and is due to Batyrev/Popov [7] and Hassett/Tschinkel [25.

The aim of the present paper is to provide computational methods for Cox rings not depending on a detailed geometric understanding of the underlying varieties. We consider modifications $X_{2} \rightarrow X_{1}$ of projective varieties, where one of the associated Cox rings $R_{1}$ and $R_{2}$ is explicitly given in terms of generators and relations, for example, $X_{1}$ might be a projective space and $X_{2} \rightarrow X_{1}$ a sequence of blow ups. Whereas $R_{1}$ can be directly determined from $R_{2}$, see Proposition 2.2 , the problem of computing $R_{2}$ from $R_{1}$ is in general delicate; even finite generation can get lost.

Our approach uses the technique of toric ambient modifications developed in [5, 26] and upgraded in Section 2 according to our computational needs. The idea is to realize $X_{2} \rightarrow X_{1}$ via a modification of toric varieties $Z_{2} \rightarrow Z_{1}$, where $X_{1} \subseteq Z_{1}$ is embedded in a compatible way, which means in particular that $R_{1}$ is the factor ring

2000 Mathematics Subject Classification. 14L24, 14L30, 14C20, 14Q10, 14Q15, 13A30, 52B55 The second author was partially supported by the DFG Priority Program SPP 1489 . The third author was partially supported by Proyecto FONDECYT Regular N. 1110096. 
of the Cox ring of $Z_{1}$ by the $\mathrm{Cl}\left(X_{1}\right)$-homogeneous ideal describing $X_{1}$. Prospective homogeneous generators $f_{j}$ for $R_{2}$ correspond either to exceptional divisors or can be encoded as prime divisors on $X_{1}$. The general basic Algorithms 3.1 and 3.6 check via the algebraic criteria 2.6 and 2.7 if a given guess of $f_{j}$ indeed generates the Cox ring $R_{2}$ and, in the affirmative case, compute the defining ideal of relations for $R_{2}$. The main computational issues are a saturation process to compute the $\mathrm{Cl}\left(X_{2}\right)$-homogeneous ideal $I_{2}$ of the proper transform $X_{2}$ in the Cox $\operatorname{ring} \mathcal{R}\left(Z_{2}\right)$ and $\mathrm{Cl}\left(X_{2}\right)$-primality tests of the $f_{j} \in \mathcal{R}\left(Z_{2}\right) / I_{2}$ to verify that $\mathcal{R}\left(Z_{2}\right) / I_{2}$ is the Cox ring $R_{2}$ of $X_{2}$ according to 2.6 and 2.7

As a first application, we consider in Section 4 the Gorenstein log del Pezzo surfaces $X$ of Picard number one; see [1, Theorem 8.3] for a classification in terms of the singularity type. The toric ones correspond to the reflexive lattice triangles, see for example [34, and their Cox rings are directly obtained by [17]. The Cox rings of the nontoric $X$ allowing still a $\mathbb{K}^{*}$-action have been determined in 28 . In Theorem 4.1, we provide the Cox rings for the remaining cases; that means for the $X$ admitting no nontrivial torus action. The approach is via a presentation $\mathbb{P}_{2} \leftarrow \widetilde{X} \rightarrow X$, where $\widetilde{X}$ is smooth. From [2, 19, 25] we infer enough information on the generators of the Cox ring of $\widetilde{X}$ for computing the Cox ring of $X$ by means of our algorithms; we note that an explicit computation of the Cox ring of $\widetilde{X}$ is not needed (and in fact was not always feasible on our systems).

The "lattice ideal method" presented in Section 5 produces systematically generators for the Cox ring of the blow up $X_{2}$ of a given Mori dream space $X_{1}$ at a subvariety $C \subseteq X_{1}$ contained in the smooth locus of $X_{1}$. The theoretical basis for this is Proposition 5.2 where we describe the Cox ring $R_{2}$ of $X_{2}$ as a saturated Rees algebra defined by the $\mathrm{Cl}\left(X_{1}\right)$-homogeneous ideal of the center $C \subseteq X_{1}$ in the Cox ring $R_{1}$ of $X_{1}$. Building on this, Algorithm 5.4 verifies a given set of prospective generators for $R_{2}$ and, in the affirmative case computes the ideal of relations of $R_{2}$; a major computational advantage compared to the more generally applicable Algorithm 3.6 is that the involved primality checks are now replaced with essentially less complex dimension computations. The $\mathbb{Z}$-grading of $R_{2}$ given by the Rees algebra structure allows to produce systematically generators of $R_{2}$ by computing stepwise generators for the $\mathbb{Z}$-homogeneous components. This is implemented in Algorithm 5.6. which basically requires the Cox ring $R_{1}$ of $X_{1}$ in terms of generators and relations and $\mathrm{Cl}\left(X_{2}\right)$-homogeneous generators of the ideal of the center of $X_{2} \rightarrow X_{1}$. It then terminates if and only if the blow up $X_{2}$ is a Mori dream space and in this case, it provides the Cox ring $R_{2}$ of $X_{2}$. A sample computation is performed in Example 5.7

An application of the lattice ideal method is given in Section 6, where we investigate smooth rational surfaces $X$ of Picard number $\varrho(X) \leq 6$. Using our algorithms, we show in Theorem 6.1 that they are all Mori dream surfaces and we provide the Cox rings for those $X$ that do not admit a nontrivial torus action; for the toric $X$ one obtains the Cox ring directly by 17 and for the $X$ with a $\mathbb{K}^{*}$-action, the methods of [28] apply. Certain blow ups of the projective plane have also been considered earlier: general point configurations lead to the smooth del Pezzo surfaces and almost general ones lead to so-called weak del Pezzo surfaces, see [7, 19, 20, 25, 43. Wheras the remaining blow ups of the plane can be settled by our methods in a purely computational way, the blow ups of Hirzebruch surfaces require besides the algorithmic also a theoretical treatment.

In Section 7, we consider blow ups of point configurations in the projective space. Algorithm 7.1 tests whether the Cox ring is generated by proper transforms of hyperplanes and, if so, computes the Cox ring. In Example 7.5 we treat the blow up of the projective plane at a symmetric configuration of seven points. Then we leave the surface case and study blow ups of the projective space $\mathbb{P}_{3}$ at configurations of six 
distinct points. Recall that in the case of general position, Castravet/Tevelev [13] determined generators of the Cox ring and Sturmfels/Xu 46, the relations, see also [45. Moreover, in [46 special configurations are considered and a certain subring of the Cox ring is described, compare [46, p. 456]. We obtain in Theorem 7.6 that the blow up of six points not contained in a hyperplane is always a Mori dream space and we list the Cox rings for the edge-special configurations, i.e. four points are general and at least one of the six lies in two hyperplanes spanned by the others.

All our algorithms are stated explicitly and will be made available within a software package. In our computations, we made intensive use of the software systems Macaulay2 [22], Magma [1], Maple [36] and Singular [18]. We would like to thank the developers for providing such helpful tools. Moreover, we are grateful to Cinzia Casagrande for her comments and discussions about Section 7. Finally, we want to express our sincere thanks to the referees for many valuable hints and helpful suggestions for improving our manuscript.

\section{Contents}

1. Introduction

2. Toric ambient modifications

3. Basic algorithms

4. Gorenstein log del Pezzo surfaces

5. The lattice ideal method

6. Smooth rational surfaces

7. Linear generation

References

\section{TORIC AMBIENT MODIFICATIONS}

As indicated before, the ground field $\mathbb{K}$ is algebraically closed and of characteristic zero throughout the article. In this section, we provide the necessary background on Cox rings, Mori dream spaces and their modifications. Let us begin with recalling notation and basics from [4. To any normal complete variety $X$ with finitely generated divisor class group $\mathrm{Cl}(X)$, one can associate a Cox sheaf and a Cox ring

$$
\mathcal{R}:=\bigoplus_{\mathrm{Cl}(X)} \mathcal{O}_{X}(D), \quad \mathcal{R}(X):=\bigoplus_{\mathrm{Cl}(X)} \Gamma\left(X, \mathcal{O}_{X}(D)\right) .
$$

The Cox ring $\mathcal{R}(X)$ is factorially $\mathrm{Cl}(X)$-graded in the sense that it is integral and every nonzero homogeneous nonunit is a product of $\mathrm{Cl}(X)$-primes. Here, a nonzero homogeneous nonunit $f \in \mathcal{R}(X)$ is called $\mathrm{Cl}(X)$-prime if for any two homogeneous $g, h \in \mathcal{R}(X)$ we have that $f \mid g h$ implies $f \mid g$ or $f \mid h$. If the divisor class group $\mathrm{Cl}(X)$ is torsion free, then the Cox $\operatorname{ring} \mathcal{R}(X)$ is even a UFD in the usual sense.

If $\mathcal{R}$ is locally of finite type, e.g. $X$ is $\mathbb{Q}$-factorial or $X$ is a Mori dream space, i.e. a normal projective variety with $\mathcal{R}(X)$ finitely generated 30 , then one has the relative spectrum $\widehat{X}:=\operatorname{Spec}_{X} \mathcal{R}$. The characteristic quasitorus $H:=\operatorname{Spec} \mathbb{K}[\operatorname{Cl}(X)]$ acts on $\widehat{X}$ and the canonical map $p: \widehat{X} \rightarrow X$ is a good quotient for this action. We call $p: \widehat{X} \rightarrow X$ a characteristic space over $X$. If $X$ is a Mori dream space, then one has the total coordinate space $\bar{X}:=\operatorname{Spec} \mathcal{R}(X)$ and a canonical $H$-equivariant open embedding $\widehat{X} \subseteq \bar{X}$. Note that the characteristic space $p: \widehat{X} \rightarrow X$ coincides with the universal torsor introduced by Colliot-Thélène and Sansuc [15, 16] if and only if $X$ is locally factorial in the sense that for every closed point $x \in X$ the local $\operatorname{ring} \mathcal{O}_{X, x}$ is a UFD. 
Given a Mori dream space $X$ and a system $\mathfrak{F}=\left(f_{1}, \ldots, f_{r}\right)$ of pairwise nonassociated $\mathrm{Cl}(X)$-prime generators of the Cox ring $\mathcal{R}(X)$, we can construct an embedding into a toric variety. First, with $\bar{Z}:=\mathbb{K}^{r}$, we have a closed embedding

$$
\bar{X} \rightarrow \bar{Z}, \quad \bar{x} \mapsto\left(f_{1}(\bar{x}), \ldots, f_{r}(\bar{x})\right) .
$$

This embedding is $H_{X}$-equivariant, where the characteristic quasitorus $H_{X}$ acts diagonally on $\bar{Z}$ via the weights $\operatorname{deg}\left(f_{i}\right) \in \mathrm{Cl}(X)=\mathbb{X}\left(H_{X}\right)$. For any ample class $w \in \mathrm{Cl}(X)$ on $X$, we obtain a set of $w$-semistable points

$$
\widehat{Z}:=\left\{\bar{z} \in \bar{Z} ; f(\bar{z}) \neq 0 \text { for some } f \in \mathbb{K}\left[T_{1}, \ldots, T_{r}\right]_{n w}, n>0\right\} .
$$

The intersection $\widehat{X}:=\bar{X} \cap \bar{Z}$ is the set of $w$-semistable points for the action of $H_{X}$ on $\bar{X}$. Altogether, this gives rise to a commutative diagram

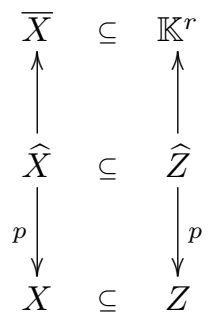

where $p: \widehat{Z} \rightarrow Z$ is the toric characteristic space [17] and we have an induced embedding $X \subseteq Z$ of quotients. Then $\mathrm{Cl}(X)=\mathrm{Cl}(Z)$ holds and $X$ inherits many geometric properties from $Z$, see [4, Sec. III.2.5] for details. We call $X \subseteq Z$ in this situation a compatibly embedded Mori dream space (CEMDS).

Remark 2.1. Consider a CEMDS $X \subseteq Z$. Let $Q: \mathbb{Z}^{r} \rightarrow K:=\mathrm{Cl}(Z)=\mathrm{Cl}(X)$ denote the degree map of the Cox ring $\mathcal{R}(Z)=\mathbb{K}\left[T_{1}, \ldots, T_{r}\right]$ of the ambient projective toric variety $Z$, sending the $i$-th canonical basis vector $e_{i} \in \mathbb{Z}^{r}$ to the degree of the $i$-th variable $T_{i}$ and let $P: \mathbb{Z}^{r} \rightarrow \mathbb{Z}^{n}$ be the Gale dual map, i.e. $P$ is dual to the inclusion $\operatorname{ker}(Q) \subseteq \mathbb{Z}^{r}$. If $w \in \mathrm{Cl}(Z)$ is an ample class of $Z$ and hence for $X$, then the fans $\widehat{\Sigma}$ of $\widehat{Z}$ and $\Sigma$ of $Z$ are given by

$$
\widehat{\Sigma}:=\left\{\widehat{\sigma} \preceq \mathbb{Q}_{\geq 0}^{r} ; w \in Q\left(\widehat{\sigma}^{\perp} \cap \mathbb{Q}_{\geq 0}^{r}\right)\right\}, \quad \Sigma^{\max }=\left\{P(\widehat{\sigma}) ; \widehat{\sigma} \in \widehat{\Sigma}^{\max }\right\},
$$

where we write $\preceq$ for the face relation of cones and regard $Q$ and $P$ as maps of the corresponding rational vector spaces. If $X \subseteq Z$ is a CEMDS, then the ample class $w \in \mathrm{Cl}(Z)=\mathrm{Cl}(X)$ is also an ample class for $X$. Note that a different choice of the ample class $w^{\prime} \in \mathrm{Cl}(X)$ may lead to another CEMDS $X \subseteq Z^{\prime}$ according to the fact that the Mori chamber decomposition of $Z$ refines the one of $X$.

We now consider modifications, that means proper birational morphisms $\pi: X_{2} \rightarrow$ $X_{1}$ of normal projective varieties. A first general statement describes the Cox ring of $X_{1}$ in terms of the Cox ring of $X_{2}$. By a morphism of graded algebras $A=\oplus_{M} A_{m}$ and $B=\oplus_{N} B_{n}$ we mean an algebra homomorphism $\psi: A \rightarrow B$ together with an accompanying homomorphism $\widetilde{\psi}: M \rightarrow N$ of the grading groups such that $\psi\left(A_{m}\right) \subseteq B_{\widetilde{\psi}(m)}$ holds for all $m \in M$.

Proposition 2.2. Let $\pi: X_{2} \rightarrow X_{1}$ be a modification of normal projective varieties and let $C \subseteq X_{1}$ be the center of $\pi$. Set $K_{i}:=\mathrm{Cl}\left(X_{i}\right)$ and $R_{i}:=\mathcal{R}\left(X_{i}\right)$ and identify $U:=X_{2} \backslash \pi^{-1}(C)$ with $X_{1} \backslash C$. Then we have canonical surjective push forward maps

$$
\tilde{\pi}_{*}: K_{2} \rightarrow K_{1},[D] \mapsto\left[\pi_{*} D\right], \quad \pi_{*}: R_{2} \rightarrow R_{1},\left(R_{2}\right)_{[D]} \ni f \mapsto f_{\mid U} \in\left(R_{1}\right)_{\left[\tilde{\pi}_{*} D\right]} .
$$

Now suppose that $\mathcal{R}\left(X_{2}\right)$ is finitely generated, let $E_{1}, \ldots, E_{l} \subseteq X_{2}$ denote the exceptional prime divisors and $f_{1}, \ldots, f_{l} \in \mathcal{R}\left(X_{2}\right)$ the corresponding canonical sections. 
Then we have a commutative diagram

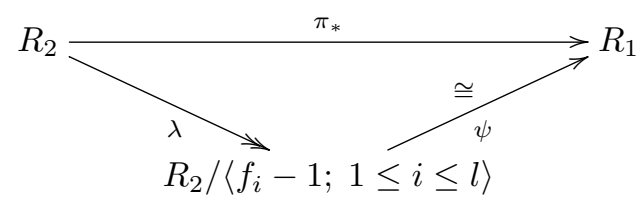

of morphisms of graded algebras, where $\lambda$ is the canonical projection with the projection $\tilde{\lambda}: K_{2} \rightarrow K_{2} /\left\langle\operatorname{deg}\left(f_{1}\right), \ldots, \operatorname{deg}\left(f_{l}\right)\right\rangle$ as accompanying homomorphism and the induced morphism $\psi$ is an isomorphism.

Lemma 2.3. Let $R$ be a $K_{2}$-graded domain, $f \in R_{w}$ with $w$ of infinite order in $K_{2}$ and consider the downgrading of $R$ given by $K_{2} \rightarrow K_{1}:=K_{2} /\langle w\rangle$. Then $f-1$ is $K_{1}$-prime.

Proof. Let $(f-1) g=a b$, where $g, a, b \in R$ are $K_{1}$-homogeneous elements. Since $w$ has infinite order, any $K_{1}$-homogeneous element $u \in R$ can be uniquely written as a sum $u=u_{0}+\ldots+u_{n}$, where each $u_{i}$ is $K_{2}$-homogeneous, both $u_{0}$ and $u_{n}$ are non-zero and $\operatorname{deg}_{K_{2}}\left(u_{i}\right)=\operatorname{deg}_{K_{2}}\left(u_{0}\right)+i w$ holds for each $i$ such that $u_{i}$ is non-zero. According to this observation we write

$$
(f-1) \sum_{i=0}^{n} g_{i}=\left(\sum_{i=0}^{n_{1}} a_{i}\right)\left(\sum_{i=0}^{n_{2}} b_{i}\right) .
$$

We have $-g_{0}=a_{0} b_{0}$ since otherwise, by equating the $K_{2}$-homogeneous elements of the same degree we would obtain either $g_{0}=0$ or $a_{0} b_{0}=0$. Similarly, we see $f g_{n}=a_{n_{1}} b_{n_{2}}$. Thus

$$
f g_{n}=a_{n_{1}} b_{n_{2}}, \quad f g_{n-1}-g_{n}=a_{n_{1}} b_{n_{2}-1}+a_{n_{1}-1} b_{n_{2}}, \quad \ldots \quad-g_{0}=a_{0} b_{0} .
$$

By an induction argument, eliminating the $g_{i}$ gives

$$
\left(a_{0} f^{n_{1}}+a_{1} f^{n_{1}-1}+\cdots+a_{n_{1}}\right)\left(b_{0} f^{n_{2}}+b_{1} f^{n_{2}-1}+\cdots+b_{n_{2}}\right)=0 .
$$

Since $R$ is integral, one of the two factors must be zero, say the first one. Then $f-1$ divides

$$
a=a_{0}+\cdots+a_{n_{1}}=a_{0}\left(1-f^{n_{1}}\right)+\cdots+a_{n_{1}-1}(1-f) .
$$

Proof of Proposition 2.2. Let $x_{i} \in X_{i}$ be smooth points with $\pi\left(x_{2}\right)=x_{1}$ such that $x_{2}$ is not contained in any of the exceptional divisors. Consider the divisorial sheaf $\mathcal{S}_{i}$ on $X_{i}$ associated to the subgroup of divisors avoiding the point $x_{i}$, see 4 , Constr. 4.2.3]. The open subset $U=X_{2} \backslash \pi^{-1}(C) \subseteq X_{2}$ is mapped by $\pi$ isomorphically onto $X_{1} \backslash C$. This leads to canonical morphisms of graded algebras

$$
\Gamma\left(X_{2}, \mathcal{S}_{2}\right) \rightarrow \Gamma\left(U_{2}, \mathcal{S}_{2}\right) \rightarrow \Gamma\left(X_{1} \backslash C, \mathcal{S}_{1}\right) \rightarrow \Gamma\left(X_{1}, \mathcal{S}_{1}\right),
$$

where the accompanying homomorphisms of the grading groups are the respective push forwards of Weil divisors; here we use that $C$ is of codimension at least two in $X_{1}$ and thus any section of $\mathcal{S}_{1}$ over $X_{1} \backslash C$ extends uniquely to a section of $\mathcal{S}_{1}$ over $X_{1}$. The homomorphisms are compatible with the relations of the Cox sheaves $\mathcal{R}_{i}$, see again [4, Constr. 4.2.3], and thus induce canonical morphisms of graded rings

$$
\Gamma\left(X_{2}, \mathcal{R}_{2}\right) \rightarrow \Gamma\left(U_{2}, \mathcal{R}_{2}\right) \rightarrow \Gamma\left(X_{1} \backslash C, \mathcal{R}_{1}\right) \rightarrow \Gamma\left(X_{1}, \mathcal{R}_{1}\right) .
$$

This establishes the surjection $\pi_{*}: R_{2} \rightarrow R_{1}$ with the canonical push forward $\tilde{\pi}_{*}$ of divisor class groups as accompanying homomorphism. Clearly, the canonical sections $f_{i}$ of the exceptional divisors are sent to $1 \in R_{1}$.

We show that the induced map $\psi$ is an isomorphism. As we may proceed by induction on $l$, it suffices to treat the case $l=1$. Lemma 2.3 tells us that $f_{1}-1$ is $K_{1}$-prime. From [26, Prop. 3.2] we infer that $\left\langle f_{1}-1\right\rangle$ is a radical ideal in $R_{2}$. Since 
$\operatorname{Spec}(\psi)$ is a closed embedding of varieties of the same dimension and equivariant with respect to the action of the quasitorus $\operatorname{Spec} \mathbb{K}\left[K_{1}\right]$, the assertion follows.

We are grateful to the referee suggesting to us the following example as a geometric illustration.

Example 2.4. Let $X_{2}$ be the blow-up of $\mathbb{P}^{2}$ at the three toric fixed points and $p=[1,1,1]$ and let $\pi: X_{2} \rightarrow X_{1}$ be the contraction of the exceptional curve $E$ over $p$. The Cox ring $R_{2}$ of $X_{2}$ is the coordinate ring of the affine cone $\bar{X}_{2}$ over $G(2,5)$, that means that $R_{2}$ is $\mathbb{K}\left[T_{1}, \ldots, T_{10}\right]$ modulo the ideal $I_{2}$ generated by the Plücker relations

$$
\begin{gathered}
T_{7} T_{8}-T_{6} T_{9}+T_{5} T_{10}, \quad T_{4} T_{6}-T_{3} T_{7}-T_{1} T_{10}, \quad T_{4} T_{8}-T_{3} T_{9}+T_{2} T_{10}, \\
T_{4} T_{5}-T_{2} T_{7}-T_{1} T_{9}, \quad T_{3} T_{5}-T_{2} T_{6}-T_{1} T_{8} .
\end{gathered}
$$

The $\mathrm{Cl}\left(X_{2}\right)$-grading is the finest one leaving variables and relations homogeneous. We assume $E$ to be $V\left(T_{10}\right)$ in Cox coordinates. According to Proposition 2.2 we have an epimorphism $R_{2} \rightarrow R_{2} /\left\langle T_{10}-1\right\rangle \cong R_{1}$ onto the Cox ring $R_{1}$ of $X_{1}$. This defines a closed embedding of $\bar{X}_{1}=\mathbb{K}^{6}$ as $\bar{X}_{2} \cap V\left(T_{10}-1\right)$ into $\bar{X}_{2} \backslash V\left(T_{10}\right) \subseteq \mathbb{K}^{10}$; this embedding is explicitly given by

$$
\left(z_{1}, \ldots, z_{6}\right) \mapsto\left(z_{2} z_{3}-z_{1} z_{4}, z_{1} z_{6}-z_{2} z_{5}, z_{1}, z_{2}, z_{3} z_{6}-z_{4} z_{5}, z_{3}, z_{4}, z_{5}, z_{6}, 1\right) .
$$

Observe that $\bar{X}_{2} \backslash V\left(T_{10}\right)$ is the subset of the affine cone over $G(2,5)$ corresponding to a Schubert cell consisting of all lines of $\mathbb{P}_{4}$ not meeting a certain plane in $\mathbb{P}_{4}$.

As an immediate consequence of Proposition 2.2. we obtain that $X_{1}$ is a Mori dream space provided $X_{2}$ is one; recall that in 40] it is more generally proven that for any dominant morphism $X_{1} \rightarrow X_{2}$ of $\mathbb{Q}$-factorial projective varieties, $X_{2}$ is a Mori dream space if $X_{1}$ is. The converse question is in general delicate. For a classical counterexample, consider points $x_{1}, \ldots, x_{9} \in \mathbb{P}_{2}$ that lie on precisely one smooth cubic $\Gamma \subseteq \mathbb{P}_{2}$ and admit a line $\ell \subseteq \mathbb{P}_{2}$ such that $\left.3 \ell\right|_{\Gamma}-p_{1}-\ldots-p_{9}$ is not a torsion element of $\operatorname{Pic}^{0}(\Gamma)$. Then the blow up $X_{1}$ of $\mathbb{P}_{2}$ at $x_{1}, \ldots, x_{8}$ is a Mori dream surface and the blow up $X_{2}$ of $X_{1}$ at $x_{9}$ is not, see for example [4, Prop. 4.3.4.5].

We now upgrade the technique of toric ambient modifications developed in [26, 5] according to our computational purposes. In the following setting, $\widehat{X}_{i} \rightarrow X_{i}$ is not necessarily a characteristic space and $\bar{X}_{i}$ not necessarily a total coordinate space.

Setting 2.5. Let $\pi: Z_{2} \rightarrow Z_{1}$ be a toric modification, i.e. $Z_{1}, Z_{2}$ are complete toric varieties and $\pi$ is a proper birational toric morphism. Moreover, let $X_{i} \subseteq Z_{i}$ be closed subvarieties, both intersecting the big $n$-torus $\mathbb{T}^{n} \subseteq Z_{i}$, such that $\pi\left(X_{2}\right)=X_{1}$ holds. Then we have a commutative diagram

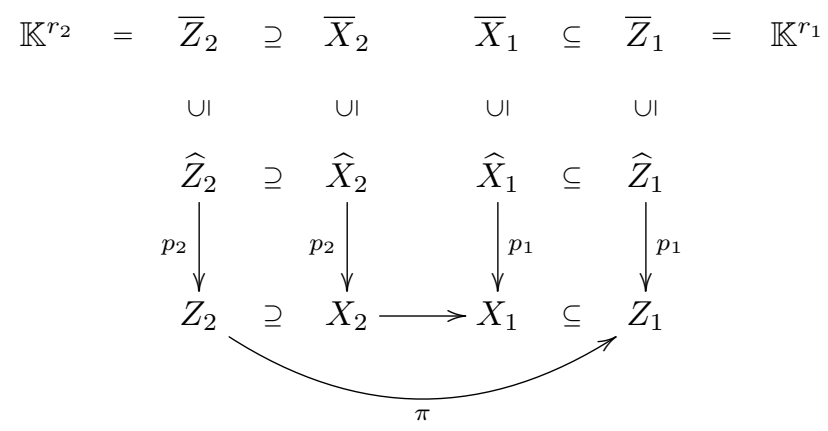

where the downwards maps $p_{i}: \widehat{Z}_{i} \rightarrow Z_{i}$ are toric characteristic spaces and $\widehat{X}_{i} \subseteq \widehat{Z}_{i}$ are the closures of the inverse image $p_{i}^{-1}\left(X_{i} \cap \mathbb{T}^{n}\right)$. Let $I_{i} \subseteq \mathbb{K}\left[T_{1}, \ldots, T_{r_{i}}\right]$ be the vanishing ideal of the closure $\bar{X}_{i} \subseteq \mathbb{K}^{r_{i}}$ of $\widehat{X}_{i} \subseteq \widehat{Z}_{i}$ and set $R_{i}:=\mathbb{K}\left[T_{1}, \ldots, T_{r_{i}}\right] / I_{i}$. Note that $R_{i}$ is graded by $K_{i}:=\mathrm{Cl}\left(Z_{i}\right)$. 
Theorem 2.6. Consider the Setting 2.5.

(i) If $X_{1} \subseteq Z_{1}$ is a CEMDS, the ring $R_{2}$ is normal and $T_{1}, \ldots, T_{r_{2}}$ define pairwise non-associated $K_{2}$-primes in $R_{2}$, then $X_{2} \subseteq Z_{2}$ is a CEMDS. In particular, $K_{2}$ is the divisor class group of $X_{2}$ and $R_{2}$ is the Cox ring of $X_{2}$.

(ii) If $X_{2} \subseteq Z_{2}$ is a CEMDS, then $X_{1} \subseteq Z_{1}$ is a CEMDS. In particular, $K_{1}$ is the divisor class group of $X_{1}$ and $R_{1}$ is the Cox ring of $X_{1}$.

Proof. First consider the lattice homomorphisms $P_{i}: \mathbb{Z}^{r_{i}} \rightarrow \mathbb{Z}^{n}$ associated to the toric morphisms $p_{i}: \widehat{Z}_{i} \rightarrow Z_{i}$. Viewing the $P_{i}$ as matrices, we may assume that $P_{2}=\left[P_{1}, B\right]$ with a matrix $B$ of size $n \times\left(r_{2}-r_{1}\right)$. We have a commutative diagram of lattice homomorphisms and the corresponding diagram of homomorphisms of tori:
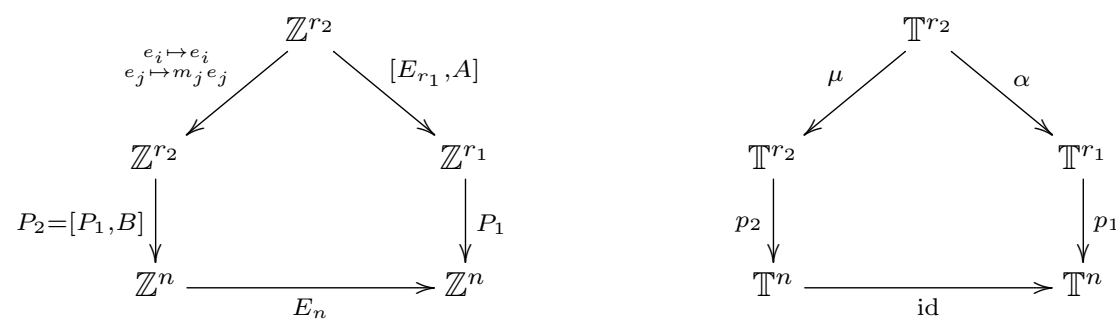

where in the left diagram, the $e_{i}$ are the first $r_{1}$, the $e_{j}$ the last $r_{2}-r_{1}$ canonical basis vectors of $\mathbb{Z}^{r_{2}}$, the $m_{j}$ are positive integers and $E_{n}, E_{r_{1}}$ denote the unit matrices of size $n, r_{1}$ respectively and $A$ is an integral $r_{1} \times\left(r_{2}-r_{1}\right)$ matrix.

We prove (i). We first show that $R_{2}$ is integral. By construction, it suffices to show that $p_{2}^{-1}\left(X_{1} \cap \mathbb{T}^{n}\right)$ is irreducible. By assumption, $\bar{X}_{1} \cap \mathbb{T}^{r_{1}}$ is irreducible. Since $\alpha$ has connected kernel, also $\alpha^{-1}\left(\bar{X}_{1} \cap \mathbb{T}^{r_{1}}\right)$ is irreducible. We conclude that $\bar{X}_{2} \cap \mathbb{T}^{r_{2}}=\mu\left(\alpha^{-1}\left(\bar{X}_{1} \cap \mathbb{T}^{r_{1}}\right)\right)$ is irreducible. Moreover, since $X_{2}$ is complete and the $K_{2}$-grading of $R_{2}$ has a pointed weight cone, we obtain that $R_{2}$ has only constant units. Thus, [5, Thm. 3.2] yields that $R_{2}$ is factorially $K_{2}$-graded. Since the $T_{i}$ are pairwise non-associated $K_{2}$-primes and $R_{2}$ is normal, we conclude that $R_{2}$ is the Cox ring of $X_{2}$ and $X_{2} \subseteq Z_{2}$ is a CEMDS.

We turn to (ii). Observe that for every $f \in I_{2}$, the Laurent polynomials $\mu^{*}(f)$ and $\alpha^{*}\left(f\left(t_{1}, \ldots, t_{r_{1}}, 1, \ldots, 1\right)\right)$ differ by a monomial factor. We conclude

$$
\mathbb{K}\left[T_{1}^{ \pm 1}, \ldots, T_{r_{2}}^{ \pm 1}\right] \cdot I_{2}=\left\langle\alpha^{*}\left(f\left(t_{1}, \ldots, t_{r_{1}}, 1, \ldots, 1\right)\right) ; f \in I_{2}\right\rangle \subseteq \mathbb{K}\left[T_{1}^{ \pm 1}, \ldots, T_{r_{2}}^{ \pm 1}\right] .
$$

Now Proposition 2.2 tells us that $R_{1}$ is the Cox ring of $X_{1}$. Since $T_{1}, \ldots, T_{r_{1}}$ define pairwise different prime divisors in $X_{1}$, we conclude that $X_{1} \subseteq Z_{1}$ is a CEMDS.

The verification of normality as well as the primality tests needed for Theorem 2.6 (ii) are computationally involved. The following observation considerably reduces the effort in many cases.

Remark 2.7. See [5, Prop. 3.3]. Consider the Setting 2.5 and assume that the canonical map $K_{2} \rightarrow K_{1}$ admits a section (e.g. $K_{1}$ is free).

(i) If $R_{1}$ is normal and $T_{r_{1}+1}, \ldots, T_{r_{2}}$ define primes in $R_{2}$ (e.g. they are $K_{2^{-}}$ prime and $K_{2}$ is free), then $R_{2}$ is normal.

(ii) Let $T_{1}, \ldots, T_{r_{1}}$ define $K_{1}$-primes in $R_{1}$ and $T_{r_{1}+1}, \ldots, T_{r_{2}}$ define $K_{2}$-primes in $R_{2}$. If no $T_{j}$ with $j \geq r_{1}+1$ divides a $T_{i}$ with $i \leq r_{1}$ in $R_{2}$, then also $T_{1}, \ldots, T_{r_{1}}$ define $K_{2}$-primes in $R_{2}$.

As a consequence of Theorem 2.6, we obtain that the modifications preserving finite generation are exactly those arising from toric modifications as discussed. More precisely, let $Z_{2} \rightarrow Z_{1}$ be a toric modification mapping $X_{2} \subseteq Z_{2}$ onto $X_{1} \subseteq Z_{1}$. We call $Z_{2} \rightarrow Z_{1}$ a good toric ambient modification if it is as in Theorem 2.6 (i). 
Corollary 2.8. Let $X_{2} \rightarrow X_{1}$ be a birational morphism of normal projective varieties such that the Cox ring $\mathcal{R}\left(X_{1}\right)$ is finitely generated. Then the following statements are equivalent.

(i) The Cox ring $\mathcal{R}\left(X_{2}\right)$ is finitely generated.

(ii) The morphism $X_{2} \rightarrow X_{1}$ arises from a good toric ambient modification.

Proof. The implication "(ii) $\Rightarrow(\mathrm{i})$ " is Theorem 2.6. For the reverse direction, set $K_{i}:=\mathrm{Cl}\left(X_{i}\right)$ and $R_{i}:=\mathcal{R}\left(X_{i}\right)$. Let $f_{1}, \ldots, f_{r_{2}}$ be pairwise nonassociated $K_{2}$-prime generators for $R_{2}$. According to Proposition 2.2, we may assume, after suitably numbering, that $f_{1}, \ldots, f_{r_{1}}$ define generators of $R_{1}$, where $r_{1} \leq r_{2}$. Now take an ample class $w_{1} \in K_{1}$. Then the pullback $w_{2}^{\prime} \in K_{2}$ of $w_{1}$ under $X_{2} \rightarrow X_{1}$ is semiample on $X_{2}$. Choose $w_{2} \in K_{2}$ such that $w_{2}$ is ample on $X_{2}$ and the toric ambient variety $Z_{2}$ of $X_{2}$ defined by $w_{2}$ has an ample cone containing $w_{2}^{\prime}$ in its closure. Then, with the sets of semistable points $\widehat{Z}_{2}, \widehat{Z}_{2}^{\prime} \subseteq \mathbb{K}^{r_{2}}$ defined by $w_{2}, w_{2}^{\prime}$ respectively and $\widehat{Z}_{1} \subseteq \mathbb{K}^{r_{1}}$ the one defined by $w_{1}$. Then we obtain morphisms

$$
Z_{2}=\widehat{Z}_{2} / / H_{2} \rightarrow \widehat{Z}_{2}^{\prime} / / H_{2} \cong \widehat{Z}_{1} / / H_{2}=Z_{1}
$$

where $H_{i}:=\operatorname{Spec} \mathbb{K}\left[K_{i}\right]$ denotes the characteristic quasitorus of $Z_{i}$; observe that $\widehat{Z}_{2}^{\prime} \rightarrow \widehat{Z}_{2}^{\prime} / / H_{2}$ is in general not a toric characteristic space. Thus, we arrive at Setting 2.5 and $Z_{2} \rightarrow Z_{1}$ is the desired good toric ambient modification inducing the morphism $X_{2} \rightarrow X_{1}$.

For a flexible use of Theorem 2.6 we will have to adjust given embeddings of a Mori dream space, e.g. bring general points of a CEMDS into a more special position, or remove linear relations from a redundant presentation of the Cox ring. The formal framework is the following.

Setting 2.9. Let $Z_{1}$ be a projective toric variety with toric total coordinate space $\bar{Z}_{1}=\mathbb{K}^{r_{1}}$, toric characteristic space $p_{1}: \widehat{Z}_{1} \rightarrow Z_{1}$ and ample class $w \in K_{1}:=$ $\mathrm{Cl}\left(Z_{1}\right)$. Consider $K_{1}$-homogeneous polynomials $h_{1}, \ldots, h_{l} \in \mathbb{K}\left[T_{1}, \ldots, T_{r_{1}}\right]$ and, with $r_{1}^{\prime}:=r_{1}+l$, the (in general non-toric) embedding

$$
\bar{\imath}: \mathbb{K}^{r_{1}} \rightarrow \mathbb{K}^{r_{1}^{\prime}}, \quad\left(z_{1}, \ldots, z_{r_{1}}\right) \mapsto\left(z_{1}, \ldots, z_{r_{1}}, h_{1}(z), \ldots, h_{l}(z)\right) .
$$

Note that $\mathbb{K}\left[T_{1}, \ldots, T_{r_{1}^{\prime}}\right]$ is graded by $K_{1}^{\prime}:=K_{1}$ via attaching to $T_{1}, \ldots, T_{r_{1}}$ their former $K_{1}$-degrees and to $T_{r_{1}+i}$ the degree of $h_{i}$. The class $w \in K_{1}^{\prime}$ defines a toric variety $Z_{1}^{\prime}$ with toric total coordinate space $\bar{Z}_{1}^{\prime}=\mathbb{K}^{r_{1}^{\prime}}$ toric characteristic space $p_{1}: \widehat{Z}_{1}^{\prime} \rightarrow Z_{1}^{\prime}$. Any closed subvariety $X_{1} \subseteq Z_{1}$ and its image $X_{1}^{\prime}:=\imath\left(X_{1}\right)$ lead to a commutative diagram

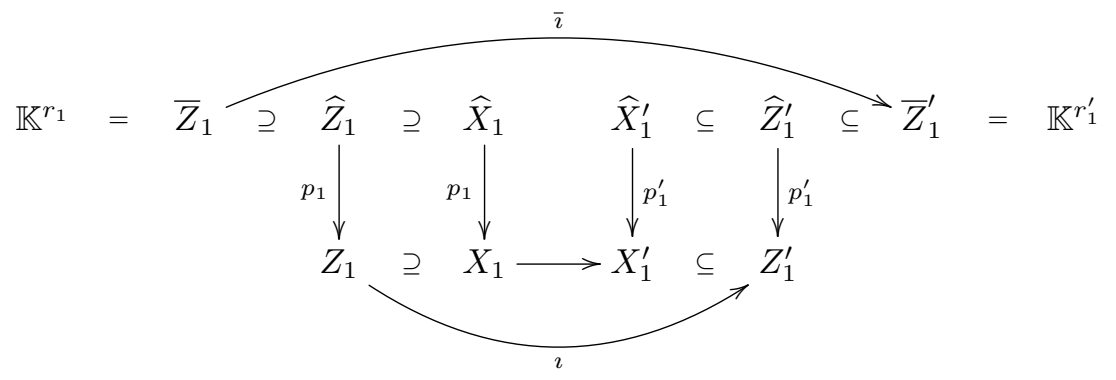

where $\widehat{X}_{1} \subseteq \widehat{Z}_{1}$ and $\widehat{X}_{1}^{\prime} \subseteq \widehat{Z}_{1}^{\prime}$ are the closures of the inverse image $p_{1}^{-1}\left(X_{1} \cap \mathbb{T}^{n}\right)$ and $\left(p_{1}^{\prime}\right)^{-1}\left(X_{1}^{\prime} \cap \mathbb{T}^{n^{\prime}}\right)$. Denote by $I_{1}$ and $I_{1}^{\prime}$ the respective vanishing ideals of the closures $\bar{X}_{1} \subseteq \mathbb{K}^{r_{1}}$ of $\widehat{X}_{1} \subseteq \widehat{Z}_{1}$ and $\bar{X}_{1}^{\prime} \subseteq \mathbb{K}^{r_{1}^{\prime}}$ of $\widehat{X}_{1}^{\prime} \subseteq \widehat{Z}_{1}^{\prime}$. Set $R_{1}:=\mathbb{K}\left[T_{1}, \ldots, T_{r_{1}}\right] / I_{1}$ and $R_{1}^{\prime}:=\mathbb{K}\left[T_{1}, \ldots, T_{r_{1}^{\prime}}\right] / I_{1}^{\prime}$.

Proposition 2.10. Consider the Setting 2.9 . 
(i) If $X_{1} \subseteq Z_{1}$ is a CEMDS and $T_{1}, \ldots, T_{r_{1}}, h_{1}, \ldots, h_{l}$ define pairwise nonassociated $K_{1}$-primes in $R_{1}$ then $X_{1}^{\prime} \subseteq Z_{1}^{\prime}$ is a CEMDS.

(ii) If $R_{1}^{\prime}$ is normal the localization $\left(R_{1}^{\prime}\right)_{T_{1} \cdots T_{r_{1}}}$ is factorially $K_{1}^{\prime}$-graded and $T_{1}, \ldots, T_{r_{1}}$ define pairwise non-associated $K_{1}$-primes in $R_{1}$ such that $K_{1}$ is generated by any $r_{1}-1$ of their degrees, then $X_{1} \subseteq Z_{1}$ is a CEMDS.

(iii) If $X_{1}^{\prime} \subseteq Z_{1}^{\prime}$ is a CEMDS, then $X_{1} \subseteq Z_{1}$ is a CEMDS.

Proof. First, observe that the ideal $I_{1}^{\prime}$ equals $I_{1}+\left\langle T_{r_{1}+1}-h_{1}, \ldots, T_{r_{1}^{\prime}}-h_{l}\right\rangle$. Consequently, we have a canonical graded isomorphism $R_{1}^{\prime} \rightarrow R_{1}$ sending $T_{r_{1}+i}$ to $h_{i}$. Assertion (i) follows directly.

We prove (ii). Since $\left(R_{1}^{\prime}\right)_{T_{1} \cdots T_{r_{1}}}$ is factorially $K_{1}^{\prime}$-graded, we obtain that $\left(R_{1}\right)_{T_{1} \cdots T_{r_{1}}}$ is factorially $K_{1}$-graded. Since $T_{1}, \ldots, T_{r_{1}}$ define $K_{1}$-primes in $R_{1}$, we can apply [8, Thm. 1.2] to see that $R_{1}$ is factorially $K_{1}$-graded. Since $T_{1}, \ldots, T_{r_{1}}$ are pairwise non-associated we conclude that $X_{1} \subseteq Z_{1}$ is a CEMDS.

We turn to (iii). According to (ii), we only have to show that any $r_{1}-1$ of the degrees of $T_{1}, \ldots, T_{r_{1}}$ generate $K_{1}$. For this, it suffices to show that each $\operatorname{deg}\left(T_{j}\right)$ for $j=r_{1}+1, \ldots, r_{1}+l$ is a linear combination of any $r_{1}-1$ of the first $r_{1}$ degrees. Since $T_{1}, \ldots, T_{r_{1}}$ generate $R_{1}$ and $T_{j}$ is not a mutiple of any $T_{i}$, we see that for any $i=1, \ldots, r_{1}$, there is a monomial in $h_{j}$ not depending on $T_{i}$. The assertion follows.

\section{BAsiC ALGORITHMS}

Here we provide the general algorithmic framework. In order to encode a compatibly embedded Mori dream space $X_{i} \subseteq Z_{i}$ and its Cox ring $R_{i}$, we use the triple $\left(P_{i}, \Sigma_{i}, G_{i}\right)$, where $P_{i}$ and $\Sigma_{i}$ are as in Remark 2.1 and $G_{i}=\left(g_{1}, \ldots, g_{s}\right)$ is a system of generators of the defining ideal $I_{i}$ of the Cox ring $R_{i}$. We call such a triple $\left(P_{i}, \Sigma_{i}, G_{i}\right)$ as well a CEMDS.

Given a CEMDS $\left(P_{i}, \Sigma_{i}, G_{i}\right)$, the total coordinate space $\bar{X}_{i}$ is the common zero set of the functions in $G_{i}$ and the degree map $Q_{i}: \mathbb{Z}^{r_{i}} \rightarrow K_{i}$ and $P_{i}$ are Gale dual to each other in the sense that $Q_{i}$ is surjective and $P_{i}$ is the dual of the inclusion $\operatorname{ker}\left(Q_{i}\right) \subseteq \mathbb{Z}^{r_{i}}$. Moreover, $p_{i}: \widehat{X}_{i} \rightarrow X_{i}$ is the restriction of the toric morphism defined by $P_{i}$. The following two algorithms implement Proposition 2.10.

Algorithm 3.1 (StretchCEMDS). Input: a CEMDS $\left(P_{1}, \Sigma_{1}, G_{1}\right)$, a list $\left(f_{1}, \ldots, f_{l}\right)$ of polynomials $f_{i} \in \mathbb{K}\left[T_{1}, \ldots, T_{r_{1}}\right]$ defining pairwise non-associated $K_{1}$-primes in $R_{1}$.

- Compute the Gale dual $Q_{1}: \mathbb{Z}^{r_{1}} \rightarrow K_{1}$ of $P_{1}$.

- Let $Q_{1}^{\prime}: \mathbb{Z}^{r_{1}+l} \rightarrow K_{1}$ be the extension of $Q_{1}$ by the degrees of $f_{1}, \ldots, f_{l}$.

- Compute the Gale dual $P_{1}^{\prime}: \mathbb{Z}^{r_{1}+l} \rightarrow \mathbb{Z}^{n^{\prime}}$ of $Q_{1}^{\prime}$ and the fan $\Sigma^{\prime}$ in $\mathbb{Z}^{n^{\prime}}$ defined by $P_{1}^{\prime}$ and the ample class $w \in K_{1}^{\prime}=K_{1}$ of $Z_{1}$.

- Set $G_{1}^{\prime}:=\left(g_{1}, \ldots, g_{s}, T_{r_{1}+1}-f_{1}, \ldots, T_{r_{1}+l}-f_{l}\right)$, where $G_{1}=\left(g_{1}, \ldots, g_{s}\right)$.

Output: the CEMDS $\left(P_{1}^{\prime}, \Sigma_{1}^{\prime}, G_{1}^{\prime}\right)$.

The input of the second algorithm is more generally an embedded space $X_{1} \subseteq Z_{1}$ that means just a closed normal subvariety intersecting the big torus. In particular, we do not care for the moment if $R_{1}$ is the Cox ring of $X_{1}$. We encode $X_{1} \subseteq Z_{1}$ as well by a triple $\left(P_{1}, \Sigma_{1}, G_{1}\right)$ and name it for short an ES. For notational reasons we write $\left(P_{1}^{\prime}, \Sigma_{1}^{\prime}, G_{1}^{\prime}\right)$ for the input.

Algorithm 3.2 (CompressCEMDS). Input: an ES $\left(P_{1}^{\prime}, \Sigma_{1}^{\prime}, G_{1}^{\prime}\right)$ such that $R_{1}^{\prime}$ is normal, the localization $\left(R_{1}^{\prime}\right)_{T_{1} \cdots T_{r_{1}}}$ is factorially $K_{1}^{\prime}$-graded and the last $l$ relations in $G_{1}^{\prime}$ are fake, i.e. of the form $f_{i}=T_{i}-h_{i}$ with $h_{i}$ not depending on $T_{i}$. Option: verify.

- Successively substitute $T_{i}=h_{i}$ in $G_{1}^{\prime}$. Set $G_{1}:=\left(f_{1}, \ldots, f_{r_{1}}\right)$, where $G_{1}^{\prime}=\left(f_{1}, \ldots, f_{r_{1}^{\prime}}\right)$ and $r_{1}:=r_{1}^{\prime}-l$. 
- Set $K_{1}:=K_{1}^{\prime}$ and let $Q_{1}: \mathbb{Z}^{r_{1}} \rightarrow K_{1}$ be the map sending $e_{i}$ to $\operatorname{deg}\left(T_{i}\right)$ for $1 \leq i \leq r_{1}$.

- Compute a Gale dual $P_{1}: \mathbb{Z}^{r_{1}} \rightarrow \mathbb{Z}^{n}$ of $Q_{1}$ and the fan $\Sigma_{1}$ in $\mathbb{Z}^{n}$ defined by $P_{1}$ and the ample class $w \in K_{1}=K_{1}^{\prime}$ of $Z_{1}^{\prime}$.

- If verify was asked then

- check if any $r_{1}-1$ of the degrees of $T_{1}, \ldots, T_{r_{1}}$ generate $K_{1}$,

- check if $\operatorname{dim}\left(I_{1}\right)-\operatorname{dim}\left(I_{1}+\left\langle T_{i}, T_{j}\right\rangle\right) \geq 2$ for all $i \neq j$,

- check if $T_{1}, \ldots, T_{r_{1}}$ define $K_{1}$-primes in $R_{1}$.

Output: the ES $\left(P_{1}, \Sigma_{1}, G_{1}\right)$. If $\left(P_{1}^{\prime}, \Sigma_{1}^{\prime}, G_{1}^{\prime}\right)$ is a CEMDS or all verifications were positive, then $\left(P_{1}, \Sigma_{1}, G_{1}\right)$ is a CEMDS. In particular, then $R_{1}$ is the Cox ring of the corresponding subvariety $X_{1} \subseteq Z_{1}$.

We turn to the algorithmic version of Theorem 2.6. We will work with the saturation of an ideal $\mathfrak{a} \subseteq \mathbb{K}\left[T_{1}, \ldots, T_{r}\right]$ with respect to an ideal $\mathfrak{b} \subseteq \mathbb{K}\left[T_{1}, \ldots, T_{r}\right]$; recall that this is the ideal

$$
\mathfrak{a}: \mathfrak{b}^{\infty}:=\left\{g \in \mathbb{K}\left[T_{1}, \ldots, T_{r}\right] ; g \mathfrak{b}^{k} \subseteq \mathfrak{a} \text { for some } k \in \mathbb{Z}_{\geq 0}\right\} \subseteq \mathbb{K}\left[T_{1}, \ldots, T_{r}\right] .
$$

In case of a principal ideal $\mathfrak{b}=\langle f\rangle$, we write $\mathfrak{a}: f^{\infty}$ instead of $\mathfrak{a}: \mathfrak{b}^{\infty}$. We say that an ideal $\mathfrak{a} \subseteq \mathbb{K}\left[T_{1}, \ldots, T_{r}\right]$ is $f$-saturated if $\mathfrak{a}=\mathfrak{a}: f^{\infty}$ holds. We will only consider saturations with respect to $f=T_{1} \cdots T_{r} \in \mathbb{K}\left[T_{1}, \ldots, T_{r}\right]$; we refer to [44, Chap. 12] for the computational aspect. Let us recall the basic properties, see also 31.

Lemma 3.3. Consider $\mathbb{K}\left[T, U^{ \pm 1}\right]$ with $T=\left(T_{1}, \ldots, T_{r_{1}}\right)$ and $U=\left(U_{1}, \ldots, U_{r_{2}-r_{1}}\right)$. For $f:=U_{1} \cdots U_{r_{2}-r_{1}} \in \mathbb{K}[U]$, one has mutually inverse bijections

$$
\begin{aligned}
\left\{\text { ideals in } \mathbb{K}\left[T, U^{ \pm 1}\right]\right\} & \longleftrightarrow\{\text { f-saturated ideals in } \mathbb{K}[T, U]\} \\
\mathfrak{a} & \mapsto \mathfrak{a} \cap \mathbb{K}[T, U] \\
\langle\mathfrak{b}\rangle_{\mathbb{K}\left[T, U^{ \pm 1}\right]} & \longleftrightarrow \mathfrak{b} .
\end{aligned}
$$

Under these maps, the prime ideals of $\mathbb{K}\left[T, U^{ \pm 1}\right]$ correspond to the $f$-saturated prime ideals of $\mathbb{K}[T, U]$.

For transferring polynomials from $\mathbb{K}\left[T_{1}, \ldots, T_{r_{1}}\right]$ to $\mathbb{K}\left[T_{1}, \ldots, T_{r_{2}}\right]$ and vice versa, we use the following operations, compare also [21]. Consider a homomorphism $\pi: \mathbb{T}^{r} \rightarrow \mathbb{T}^{n}$ of tori and its kernel $H \subseteq \mathbb{T}^{r}$.

- By a $\sharp$-pull back of $g \in \mathbb{K}\left[S_{1}^{ \pm 1}, \ldots, S_{n}^{ \pm 1}\right]$ we mean a polynomial $\pi^{\sharp} g \in$ $\mathbb{K}\left[T_{1}, \ldots, T_{r}\right]$ with coprime monomials such that $\pi^{*} g$ and $\pi^{\sharp} g$ are associated in $\mathbb{K}\left[T_{1}^{ \pm 1}, \ldots, T_{r}^{ \pm 1}\right]$.

- By a $\sharp$-push forward of an $H$-homogeneous $h \in \mathbb{K}\left[T_{1}^{ \pm 1}, \ldots, T_{r}^{ \pm 1}\right]$ we mean a polynomial $\pi_{\sharp} h \in \mathbb{K}\left[S_{1}, \ldots, S_{n}\right]$ with coprime monomials such that $h$ and $\pi^{*} \pi_{\sharp} h$ are associated in $\mathbb{K}\left[T_{1}^{ \pm 1}, \ldots, T_{r}^{ \pm 1}\right]$.

Note that $\sharp$-pull backs and $\sharp$-push forwards always exist and are unique up to constants. The $\sharp$-pull back $\pi^{\sharp} g$ of a Laurent polynomial is its usual pull back $\pi^{*} g$ scaled with a suitable monomial. To compute the $\sharp$-push forward, factorize the describing $m \times n$ matrix of $\pi$ as $P=W \cdot D \cdot V$, where $W, V$ are invertible and $D$ is in Smith normal form. Then push forward with respect to the homomorphisms corresponding to the factors.

Lemma 3.4. Consider a monomial dominant morphism $\pi: \mathbb{K}^{n_{1}} \times \mathbb{T}^{n_{2}} \rightarrow \mathbb{K}^{m}$. Write $T=\left(T_{1}, \ldots, T_{n_{1}}\right)$ and $U=\left(U_{1}, \ldots, U_{n_{2}}\right)$.

(i) If $\mathfrak{a} \subseteq \mathbb{K}\left[T, U^{ \pm 1}\right]$ is a prime ideal, then $\left\langle\pi_{\sharp} \mathfrak{a}\right\rangle \subseteq \mathbb{K}\left[S^{ \pm 1}\right]$ is a prime ideal.

(ii) If $\mathfrak{b} \subseteq \mathbb{K}\left[S^{ \pm 1}\right]$ is a radical ideal, then $\left\langle\pi^{\sharp} \mathfrak{b}\right\rangle \subseteq \mathbb{K}\left[T, U^{ \pm 1}\right]$ is a radical ideal.

Proof. The first statement follows from $\left\langle\pi_{\sharp} \mathfrak{a}\right\rangle=\left(\pi^{*}\right)^{-1}(\mathfrak{a})$. To prove (ii), let $f \in$ $\sqrt{\left\langle\pi^{\sharp} \mathfrak{b}\right\rangle}$. Since $\sqrt{\left\langle\pi^{\sharp} \mathfrak{b}\right\rangle}=I\left(\pi^{-1}(V(\mathfrak{b}))\right)$ is invariant under $H:=\operatorname{ker}\left(\left.\pi\right|_{\mathbb{T}^{n_{1}}+n_{2}}\right)$, we may assume that $f$ is $H$-homogeneous, i.e. $f(h \cdot z)=\chi(h) f(z)$ holds with 
some character $\chi \in \mathbb{X}(H)$. Choose $\eta \in \mathbb{X}\left(\mathbb{T}^{n_{1}+n_{2}}\right)$ with $\chi=\eta_{\mid H}$. Then $\eta^{-1} f$ is $H$-invariant and thus belongs to $\pi^{*}(I(V(\mathfrak{b})))$. Hilbert's Nullstellensatz and the assumption give $\pi^{*}(I(V(\mathfrak{b})))=\pi^{*}(\mathfrak{b})$. We conclude $f \in\left\langle\pi^{\sharp} \mathfrak{b}\right\rangle$.

We are ready for the algorithm treating the contraction problem. We enter a weak $C E M D S\left(P_{2}, \Sigma_{2}, G_{2}\right)$ in the sense that $G_{2}$ provides generators for the extension of $I_{2}$ to $\mathbb{K}\left[T_{1}^{ \pm 1}, \ldots, T_{r_{2}}^{ \pm 1}\right]$ and a toric contraction $Z_{2} \rightarrow Z_{1}$, encoded by $\left(P_{1}, \Sigma_{1}\right)$.

Algorithm 3.5 (ContractCEMDS). Input: a weak CEMDS $\left(P_{2}, \Sigma_{2}, G_{2}\right)$ and a pair $\left(P_{1}, \Sigma_{1}\right)$, where $P_{2}=\left[P_{1}, B\right]$ and $\Sigma_{1}$ is a coarsening of $\Sigma_{2}$ removing the rays through the columns of $B$.

- Set $h_{i}:=g_{i}\left(T_{1}, \ldots, T_{r_{1}}, 1, \ldots, 1\right) \in \mathbb{K}\left[T_{1}, \ldots, T_{r_{1}}\right]$, where $G_{2}=\left(g_{1}, \ldots, g_{s}\right)$.

- Compute a system of generators $G_{1}^{\prime}$ for $I_{1}^{\prime}:=\left\langle h_{1}, \ldots, h_{s}\right\rangle:\left(T_{1} \cdots T_{r_{1}}\right)^{\infty}$.

- Set $\left(P_{1}^{\prime}, \Sigma_{1}^{\prime}, G_{1}^{\prime}\right):=\left(P_{1}, \Sigma_{1}, G_{1}^{\prime}\right)$ and reorder the variables such that the last $l$ relations of $G_{1}^{\prime}$ are as in Algorithm 3.2 .

- Apply Algorithm 3.2 to $\left(P_{1}^{\prime}, \Sigma_{1}^{\prime}, G_{1}^{\prime}\right)$ and write $\left(P_{1}, \Sigma_{1}, G_{1}\right)$ for the output.

Output: $\left(P_{1}, \Sigma_{1}, G_{1}\right)$. This is a CEMDS. In particular, $R_{1}$ is the Cox ring of the image $X_{1} \subseteq Z_{1}$ of $X_{2} \subseteq Z_{2}$ under $Z_{2} \rightarrow Z_{1}$.

Proof. First we claim that in $\mathbb{K}\left[T_{1}^{ \pm 1}, \ldots, T_{r_{1}}^{ \pm 1}\right]$, the ideal generated by $h_{1}, \ldots, h_{s}$ coincides with the ideal generated by $p_{1}^{\sharp}\left(p_{2}\right)_{\sharp} g_{1}, \ldots, p_{1}^{\sharp}\left(p_{2}\right)_{\sharp} g_{r}$. To see this, consider $p_{i}: \mathbb{T}^{r_{i}} \rightarrow \mathbb{T}^{n}$ and let $S_{1}, \ldots, S_{n}$ be the variables on $\mathbb{T}^{n}$. Then the claim follows from $\left(P_{2}\right)_{i j}=\left(P_{1}\right)_{i j}$ for $j \leq r_{1}$ and

$$
p_{2}^{*}\left(S_{i}\right)=T_{1}^{\left(P_{2}\right)_{i 1}} \cdots T_{r_{2}}^{\left(P_{2}\right)_{i r_{2}}}, \quad p_{1}^{*}\left(S_{i}\right)=T_{1}^{\left(P_{1}\right)_{i 1}} \cdots T_{r_{1}}^{\left(P_{1}\right)_{i r_{1}}} .
$$

As a consequence of the claim, we may apply Lemma 3.4 and obtain that $G_{1}^{\prime}$ defines a radical ideal in $\mathbb{K}\left[T_{1}^{ \pm}, \ldots, T_{r_{1}}^{ \pm}\right]$. Moreover, from Theorem 2.6 we infer that $\widehat{X}_{1}^{\prime}$, defined as in Setting 2.5, is irreducible. Since $G_{1}^{\prime}$ has $\widehat{X}_{1}^{\prime} \cap \mathbb{T}^{r_{1}}$ as its zero set, it defines a prime ideal in $\mathbb{K}\left[T_{1}^{ \pm}, \ldots, T_{r_{1}}^{ \pm}\right]$. Lemma 3.3 then shows that $I_{1}^{\prime} \subseteq$ $\mathbb{K}\left[T_{1}, \ldots, T_{r_{1}}\right]$ is a prime ideal. Using Theorem 2.6 again, we see that $\left(P_{1}^{\prime}, \Sigma_{1}^{\prime}, G_{1}^{\prime}\right)$ as defined in the third step of the algorithm is a CEMDS. Thus, we may enter Algorithm 3.2 and end up with a CEMDS.

We turn to the modification problem. Given a Mori dream space $X_{1}$ with Cox ring $R_{1}$ and a modification $X_{2} \rightarrow X_{1}$, we want to know if $X_{2}$ is a Mori dream space, and if so, we ask for the Cox ring $R_{2}$ of $X_{2}$. Our algorithm verifies a guess of prospective generators for $R_{2}$ and, if successful, computes the relations. In practice, the generators are added via Algorithm 3.1

Algorithm 3.6 (ModifyCEMDS). Input: a weak CEMDS $\left(P_{1}, \Sigma_{1}, G_{1}\right)$, a pair $\left(P_{2}, \Sigma_{2}\right)$ with a matrix $P_{2}=\left[P_{1}, B\right]$ and a fan $\Sigma_{2}$ having the columns of $P_{2}$ as its primitive generators and refining $\Sigma_{1}$. Options: verify.

- Compute $G_{2}^{\prime}:=\left(h_{1}, \ldots, h_{s}\right)$, where $h_{i}=p_{2}^{\sharp}\left(p_{1}\right)_{\sharp}\left(g_{i}\right)$ and $G_{1}=\left(g_{1}, \ldots, g_{s}\right)$.

- Compute a system of generators $G_{2}$ of $I_{2}:=\left\langle h_{1}, \ldots, h_{s}\right\rangle:\left(T_{r_{1}+1} \cdots T_{r_{2}}\right)^{\infty}$.

- If verify was asked then

- compute a Gale dual $Q_{2}: \mathbb{Z}^{r_{2}} \rightarrow K_{2}$ of $P_{2}$,

- check if $\operatorname{dim}\left(I_{2}\right)-\operatorname{dim}\left(I_{2}+\left\langle T_{i}, T_{j}\right\rangle\right) \geq 2$ for all $i \neq j$

- check if $T_{1}, \ldots, T_{r_{2}}$ define $K_{2}$-primes in $R_{2}$.

- check if $R_{2}$ is normal, e.g. using Remark 2.7 .

Output: $\left(P_{2}, \Sigma_{2}, G_{2}\right)$, if the verify-checks were all positive, this is a CEMDS. In particular, then $R_{2}$ is the Cox ring of the strict transform $X_{2} \subseteq Z_{2}$ of $X_{1} \subseteq Z_{1}$ with respect to $Z_{2} \rightarrow Z_{1}$.

Proof. We write shortly $\mathbb{K}\left[T, U^{ \pm 1}\right]$ with the tuples $T=\left(T_{1}, \ldots, T_{r_{1}}\right)$ and $U=$ $\left(U_{1}, \ldots, U_{r_{2}-r_{1}}\right)$ of variables. Lemma 3.4 ensures that $G_{2}$ generates a radical ideal in $\mathbb{K}\left[T, U^{ \pm}\right]$. To see that the zero set $V\left(G_{2}\right) \subseteq \mathbb{K}^{r_{1}} \times \mathbb{T}^{r_{2}-r_{1}}$ is irreducible, consider 
the situation of equation (1) in the proof of Theorem 2.6. There, in the right hand side diagram, we may lift the homomorphisms of tori to

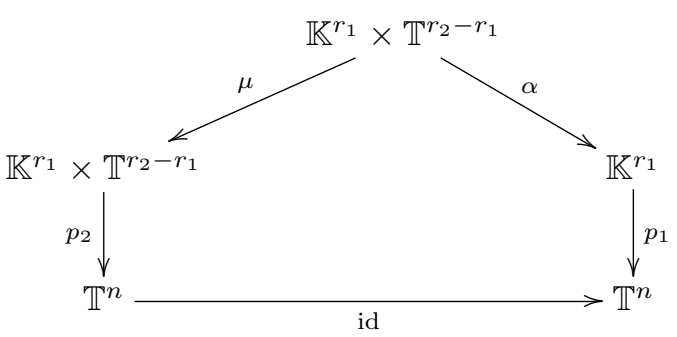

Observe that we have an isomorphism $\varphi=\alpha \times$ id given by

$$
\mathbb{K}^{r_{1}} \times \mathbb{T}^{r_{2}-r_{1}} \rightarrow \mathbb{K}^{r_{1}} \times \mathbb{T}^{r_{2}-r_{1}}, \quad\left(z, z^{\prime}\right) \mapsto\left(z_{1}\left(z^{\prime}\right)^{A_{1 *}}, \ldots, z_{r_{1}}\left(z^{\prime}\right)^{A_{r_{1 *}}}, z^{\prime}\right) .
$$

Since $\bar{X}_{1}$ is irreducible, so is $\varphi^{-1}\left(\bar{X}_{1} \times \mathbb{T}^{r_{2}-r_{1}}\right)=\alpha^{-1}\left(\bar{X}_{1}\right)$. Hence, the image $\mu\left(\alpha^{-1}\left(\bar{X}_{1}\right)\right)=\bar{X}_{2} \cap \mathbb{K}^{r_{1}} \times \mathbb{T}^{r_{2}-r_{1}}=V\left(G_{2}\right)$ is irreducible as well. Moreover, Lemma 3.3 implies that $G_{2}$ generates a prime ideal in $\mathbb{K}[T, U]$. If the verify-checks were all positive, then Theorem 2.6 tells us that $\left(P_{2}, \Sigma_{2}, G_{2}\right)$ is a CEMDS.

Remark 3.7. Compare Remark 2.7. If the canonical map $K_{2} \rightarrow K_{1}$ admits a section, e.g. if $K_{1}$ is free, then it suffices to check the variables $T_{r_{1}+1}, \ldots, T_{r_{2}}$ for $K_{2}$-primeness in $R_{2}$ in the verification step of Algorithm 3.6 .

Remark 3.8 (Verify $K$-primality). Let $\mathbb{K}\left[T_{1}, \ldots, T_{r}\right]$ be graded by a finitely generated abelian group $K$ and $I$ a $K$-homogeneous ideal. By [26, Prop. 3.2], $T_{k}$ being $K$-prime in $\mathbb{K}\left[T_{1}, \ldots, T_{r}\right] / I$ is equivalent to the divisor of $T_{k}$ in $V(I) \subseteq \mathbb{K}^{r}$ being $H:=\operatorname{Spec} \mathbb{K}[K]$-prime. In computational terms, this means computing the prime components of $I+\left\langle T_{k}\right\rangle$ and testing whether $H$ permutes them transitively. See 14, 10, 23, for the computational background. There are also recent methods from numerical algebraic geometry 42 .

\section{Gorenstein log del Pezzo surfaces}

As an application of Algorithm 3.5. we compute Cox rings of Gorenstein log terminal del Pezzo surfaces $X$ of Picard number one. Here, "del Pezzo" means that $X$ is a normal projective surface with ample anticanonical divisor $-K_{X}$ and the conditions "Gorenstein" and "log terminal" together are equivalent to saying that $X$ has at most canonical singularities which in turn are precisely the rational double points (also called ADE or du Val singularities), see for example [37, Thm. 4-6-7].

A classification of Gorenstein log terminal del Pezzo surfaces $X$ of Picard number one according to the singularity type, i.e. the configuration $\mathrm{S}(X)$ of singularities, has been given in [1, Theorem 8.3]. Besides $\mathbb{P}_{2}$, there are four toric surfaces, namely the singularity types $A_{1}, A_{1} A_{2}, 2 A_{1} A_{3}$ and $3 A_{2}$. Moreover, there are thirteen (deformation types of) $\mathbb{K}^{*}$-surfaces; they represent the singularity types $A_{4}, D_{5}$, $E_{6}, A_{1} 2 A_{3}, 3 A_{1} D_{4}, A_{1} D_{6}, A_{2} A_{5}, E_{7}, A_{1} E_{7}, A_{2} E_{6}, E_{8}, 2 D_{4}$ and their Cox rings have been determined in [28, Theorem 5.6].

We now compute the Cox rings of the remaining ones using Algorithm 3.1 and the knowledge of generators of their resolutions 19, 2, note that the relations for Cox rings of the resolutions are still unknown in some of the cases. In the sequel, we will write a Cox ring as a quotient $\mathbb{K}\left[T_{1}, \ldots, T_{r}\right] / I$ and specify generators for the ideal $I$. The $\mathrm{Cl}(X)$-grading is encoded by a degree matrix, i.e. a matrix with $\operatorname{deg}\left(T_{1}\right), \ldots, \operatorname{deg}\left(T_{r}\right) \in \operatorname{Cl}(X)$ as columns.

Theorem 4.1. The following table lists the Cox rings of the Gorenstein log terminal del Pezzo surfaces $X$ of Picard number one that do not allow a non-trivial $\mathbb{K}^{*}$-action. 
$\mathbb{K}\left[T_{1}, \ldots, T_{6}\right] / I$ with I generated by

$2 A_{4}$

$-T_{2} T_{5}+T_{3} T_{4}+T_{6}^{2},-T_{2} T_{4}+T_{3}^{2}+T_{5} T_{6}$

$T_{1} T_{6}-T_{3} T_{5}+T_{4}^{2}, T_{1} T_{3}-T_{4} T_{6}+T_{5}^{2}$,

$T_{1} T_{2}-T_{3} T_{6}+T_{4} T_{5}$

\begin{tabular}{llll}
\hline & & & \\
$D_{8}$ & $\mathbb{K}\left[T_{1}, \ldots, T_{4}\right] / I$ with I generated by & $\mathbb{Z} \oplus \mathbb{Z} / 2 \mathbb{Z}$ \\
& $T_{1}^{2}-T_{4}^{2} T_{2} T_{3}+T_{4}^{4}+T_{3}^{4}$ & {$\left[\begin{array}{cccc}2 & 1 & 1 & 1 \\
\overline{1} & \overline{1} & \overline{1} & \overline{0}\end{array}\right]$} \\
\hline & & \\
& & & \\
$D_{5} A_{3}$ & $\mathbb{K}\left[T_{1}, \ldots, T_{5}\right] / I$ with I generated by & $\mathbb{Z} \oplus \mathbb{Z} / 4 \mathbb{Z}$ \\
& $T_{1} T_{3}-T_{4}^{2}-T_{5}^{2}, T_{1} T_{2}-T_{3}^{2}+T_{4} T_{5}$ & {$\left[\begin{array}{lllll}\frac{1}{2} & \frac{1}{2} & \frac{1}{0} & \frac{1}{3} & \frac{1}{1}\end{array}\right]$}
\end{tabular}

$D_{6} 2 A_{1}$

$\mathbb{K}\left[T_{1}, \ldots, T_{5}\right] / I$ with I generated by

$\mathbb{Z} \oplus \mathbb{Z} / 2 \mathbb{Z} \oplus \mathbb{Z} / 2 \mathbb{Z}$

$T_{5} T_{2}-T_{5}^{2}+T_{3}^{2}+T_{4}^{2},-T_{2}^{2}+T_{5} T_{2}+T_{1}^{2}-T_{4}^{2}$

$\left[\begin{array}{lllll}1 & 1 & 1 & 1 & 1 \\ \overline{1} & \overline{0} & \overline{0} & \overline{1} & \overline{0} \\ \overline{0} & \overline{1} & \overline{0} & \overline{1} & \overline{1}\end{array}\right]$

\begin{tabular}{|c|c|c|c|}
\hline \multirow{2}{*}{$E_{6} A_{2}$} & $\mathbb{K}\left[T_{1}, \ldots, T_{4}\right] / I$ with I generated by & \multicolumn{2}{|l|}{$\mathbb{Z} \oplus \mathbb{Z} / 3 \mathbb{Z}$} \\
\hline & $-T_{1} T_{4}^{2}+T_{2}^{3}+T_{2} T_{3} T_{4}+T_{3}^{3}$ & {$\left[\begin{array}{lll}\frac{1}{1} & \frac{1}{2} & \frac{1}{0}\end{array}\right.$} & $\left.\frac{1}{1}\right]$ \\
\hline
\end{tabular}

$\begin{array}{llll} & \mathbb{K}\left[T_{1}, \ldots, T_{4}\right] / I \text { with I generated by } & \mathbb{Z} \oplus \mathbb{Z} / 2 \mathbb{Z} \\ E_{7} A_{1} & -T_{1} T_{3}^{3}-T_{2}^{2}+T_{2} T_{3} T_{4}+T_{4}^{4} & {\left[\begin{array}{cccc}1 & 2 & 1 & 1 \\ \overline{1} & \overline{1} & \overline{1} & \overline{0}\end{array}\right]}\end{array}$

$\begin{array}{llll}E_{8} & \mathbb{K}\left[T_{1}, \ldots, T_{4}\right] / I \text { with I generated by } & \mathbb{Z} \\ & T_{1}^{3}+T_{1}^{2} T_{4}^{2}+T_{2}^{2}-T_{3} T_{4}^{5} & {\left[\begin{array}{llll}2 & 3 & 1 & 1\end{array}\right]}\end{array}$

\begin{tabular}{lll}
\hline & $\mathbb{K}\left[T_{1}, \ldots, T_{4}\right] / I$ with I generated by & $\mathbb{Z} \oplus \mathbb{Z} / 2 \mathbb{Z}$ \\
$A_{7} \quad$ & {$\left[\begin{array}{cccc}2 & 2 & 1 & 1 \\
\overline{1} & \overline{1} & \overline{1} & \overline{0}\end{array}\right]$} \\
& $T_{1}^{2}-T_{4} T_{2} T_{3}+T_{4}^{4}+T_{3}^{4}$ &
\end{tabular}

\begin{tabular}{llll}
\hline & & & \\
$A_{8}$ & $\mathbb{K}\left[T_{1}, \ldots, T_{4}\right] / I$ with I generated by & $\mathbb{Z} \oplus \mathbb{Z} / 3 \mathbb{Z}$ & \\
& $-T_{1} T_{2} T_{3}+T_{2}^{3}+T_{3}^{3}+T_{4}^{3}$ & {$\left[\begin{array}{cccc}\frac{1}{1} & \frac{1}{2} & \overline{1} & \frac{1}{1}\end{array}\right]$} \\
\hline \multirow{3}{*}{$A_{7} A_{1}$} & $\mathbb{K}\left[T_{1}, \ldots, T_{5}\right] / I$ with I generated by & $\mathbb{Z} \oplus \mathbb{Z} / 4 \mathbb{Z}$ & \\
& $-T_{2} T_{3}+T_{4}^{2}-T_{5}^{2}, T_{1}^{2}-T_{3}^{2}+T_{4} T_{5}$ & {$\left[\begin{array}{ccccc}\frac{1}{2} & \frac{1}{2} & \frac{1}{0} & \frac{1}{3} & \frac{1}{1}\end{array}\right]$} \\
\hline
\end{tabular}

$\mathbb{K}\left[T_{1}, \ldots, T_{7}\right] / I$ with I generated by

$T_{5}^{2}+T_{6}^{2}-T_{7} T_{1}, T_{4} T_{5}+T_{6} T_{1}-T_{2} T_{6}-T_{7}^{2}, \quad T_{1} T_{3}-T_{2} T_{3}+T_{6} T_{5}-T_{7} T_{4}$,

$T_{1} T_{2}-T_{2}^{2}-T_{4}^{2}+T_{3} T_{5}$

$A_{5} A_{2} A_{1} T_{1} T_{5}-T_{2} T_{5}-T_{4} T_{6}+T_{7} T_{3}$,

$T_{1} T_{2}-T_{2}^{2}-T_{4}^{2}+T_{3} T_{5}$
$T_{1}^{2}-T_{2}^{2}-T_{4}^{2}+2 T_{3} T_{5}-T_{7} T_{6}$

$T_{3} T_{4}-T_{6}^{2}+T_{7} T_{1}-T_{2} T_{7}$,

The class group and degree matrix are $\left[\begin{array}{lllllll}\frac{1}{2} & \frac{1}{2} & \frac{1}{3} & \frac{1}{5} & \frac{1}{1} & \frac{1}{4} & \frac{1}{0}\end{array}\right]$
$\mathbb{Z} \oplus \mathbb{Z} / 6 \mathbb{Z}$ 


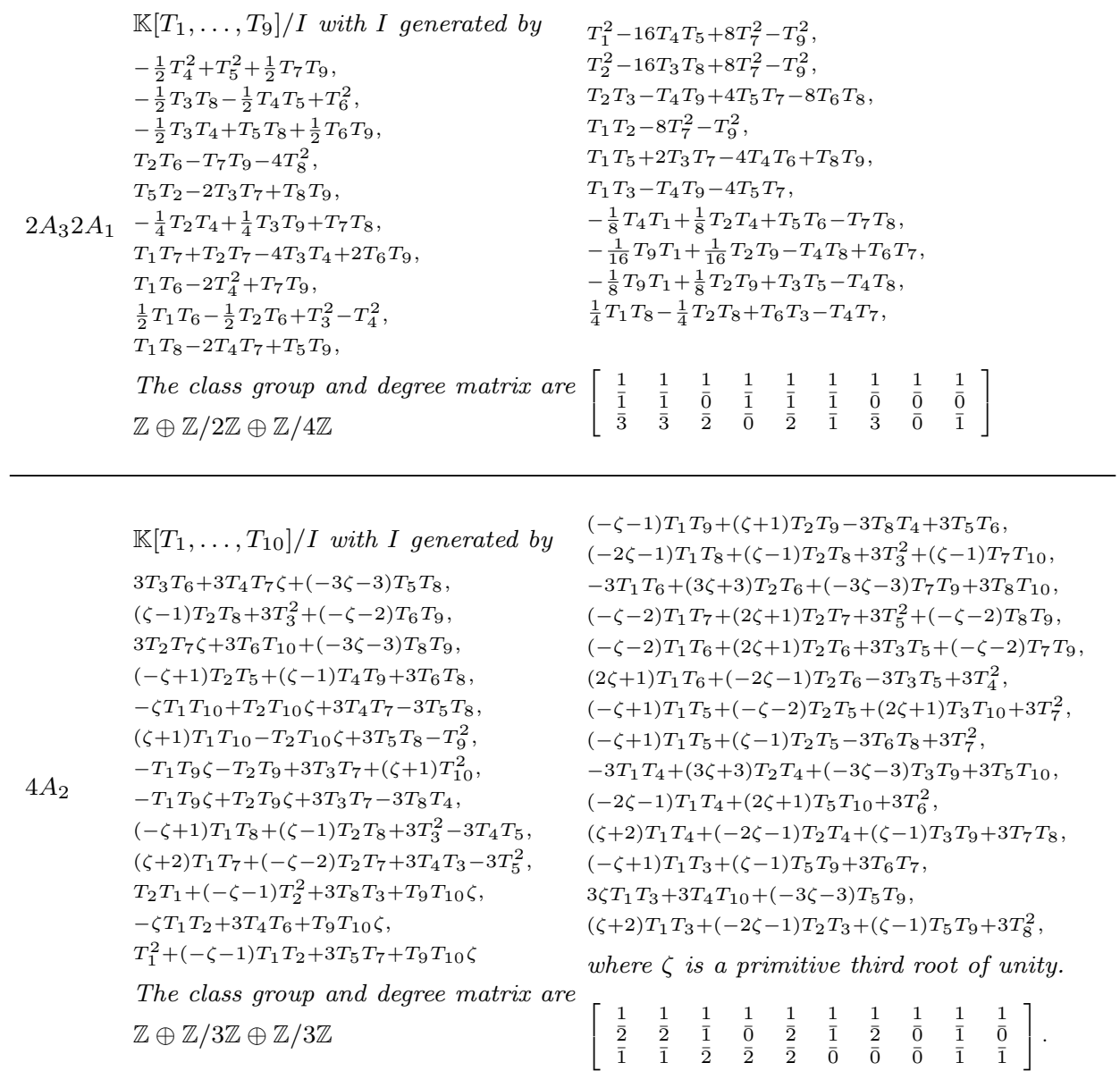

Proof. First recall from [1, Theorem 8.3] that the isomorphy classes of Gorenstein log del Pezzo surfaces of Picard number one that do not allow a $\mathbb{K}^{*}$-action correspond bijectively to singularity types we listed.

Each surface $X$ of the list is obtained by contracting curves of a smooth surface $X_{2}$ arising as a blow up of $\mathbb{P}_{2}$ with generators for the Cox ring known by [19, 2]. A direct application of Algorithms 3.6 and 3.5 is not always feasible. However, we have enough information to present the blow ups of $\mathbb{P}_{2}$ as a weak CEMDS. As an example, we treat the $D_{5} A_{3}$-case. By [2, with $X_{2}:=X_{141}$, additional generators for $\mathcal{R}\left(X_{2}\right)$ correspond in $\mathcal{R}\left(\mathbb{P}_{2}\right)$ to

$$
f_{1}:=T_{1}-T_{2}, \quad f_{2}:=T_{1} T_{2}-T_{2}^{2}+T_{1} T_{3} .
$$

Using Algorithm 3.1 with input the CEMDS $\mathbb{P}_{2}$ and $\left(f_{1}, f_{2}\right)$, we obtain a CEMDS $X_{1}$. Again by [2, Sec. 6], we know the degree matrix $Q_{2}$ of $X_{2}$. Write $Q_{2}=[D, C]$ with submatrices $D$ and $C$ consisting of the first $r_{1}$ and the last $r_{2}-r_{1}$ columns respectively. We compute a Gale dual matrix $P_{2}$ of the form $P_{2}=\left[P_{1}, B\right]$ by solving $C B^{t}=-D P_{1}^{t}$. Let $p_{1}: \mathbb{T}^{5} \rightarrow \mathbb{T}^{4}$ and $p_{2}: \mathbb{T}^{14} \rightarrow \mathbb{T}^{4}$ be the maps of tori corresponding to $P_{1}$ and $P_{2}$. Instead of using Algorithm 3.6. we directly produce the equations $G_{2}^{\prime}$ for $X_{2}$ on the torus:

$$
\begin{aligned}
& p_{2}^{\sharp}\left(p_{1}\right)_{\sharp} f_{1}=T_{1} T_{6} T_{7} T_{8} T_{14}-T_{2} T_{10} T_{11}^{2}-T_{3} T_{12} T_{13}^{2}, \\
& p_{2}^{\sharp}\left(p_{1}\right)_{\sharp} f_{2}=T_{1} T_{4} T_{14}^{2}+T_{2} T_{3} T_{9} T_{11} T_{13}-T_{5} T_{6}^{2} T_{7} .
\end{aligned}
$$

Note that by [2], the variables define pairwise non-associated $\mathrm{Cl}\left(X_{2}\right)$-prime generators for $\mathcal{R}\left(X_{2}\right)$. This makes $X_{2}$ a weak CEMDS with data $\left(P_{2}, \Sigma_{2}, G_{2}^{\prime}\right)$, where $\Sigma_{2}$ 
is the stellar subdivision of the fan $\Sigma_{1}$ of the CEMDS $X_{1}$ at the columns of $B$. We now use Algorithm 3.5 to contract on $X_{2}$ the curves corresponding to the variables $T_{i}$ with $i \in\{2,3,5,7,8,9,10,12,14\}$. The resulting ring is the one listed in the table of the theorem.

Remark 4.2. The minimal resolutions $\tilde{X}$ of the surfaces $X$ listed in Theorem 4.1 arise from the plane $\mathbb{P}_{2}$ by blowing up $9-d$ points in almost general position, where $d$ is the degree of the weak del Pezzo surface $\widetilde{X}$. In the case of singularity type $A_{7}$ we obtain degree 2 and in all other cases we have degree 1.

Remark 4.3. The surfaces with singularity type $E_{6} A_{2}, E_{7} A_{1}$ and $E_{8}$ are the only ones in the list Theorem 4.1 for which the minimal resolution has a hypersurface as Cox ring, see [19, Sect. 3, Table 9]. Moreover, these surfaces admit small degenerations into $\mathbb{K}^{*}$-surfaces. In fact, multiplying the monomials $T_{2} T_{3} T_{4}$ and $T_{1}^{2} T_{4}^{2}$ in the respective Cox rings with a parameter $\alpha \in \mathbb{K}$ gives rise to a flat family of Cox rings over $\mathbb{K}$. The induced flat family of surfaces over $\mathbb{K}$ has a $\mathbb{K}^{*}$-surface as zero fiber, compare also the corresponding Cox rings listed in [28, Theorem 5.6].

Remark 4.4. The surfaces of singularity type $A_{7}, A_{8}$ and $D_{8}$ have hypersurface Cox rings but their resolutions do not, see again [19, Sect. 3, Table 9]. For example, the Cox ring of the minimal resolution of the surface with a $D_{8}$ singularity is $\mathbb{K}\left[T_{1}, \ldots, T_{14}\right] / I$, where $I$ is generated by

$$
\begin{array}{cc}
T_{1} T_{2} T_{11}^{4} T_{12}^{4} T_{13}^{3} T_{14}^{2}+T_{3}^{2} T_{10}-T_{4} T_{7}^{2} T_{8}, & T_{1} T_{4}+T_{2}^{3} T_{7}^{2} T_{8}^{3} T_{9}^{4} T_{10}^{2} T_{13} T_{14}^{2}-T_{6} T_{11}, \\
T_{1}^{2} T_{2} T_{11}^{3} T_{12}^{4} T_{13}^{3} T_{14}^{2}+T_{5} T_{10}-T_{6} T_{7}^{2} T_{8}, & T_{1} T_{3}^{2}+T_{2}^{3} T_{7}^{4} T_{8}^{4} T_{9}^{4} T_{10} T_{13} T_{14}^{2}-T_{5} T_{11}, \\
T_{1} T_{2}^{4} T_{7}^{2} T_{8}^{3} T_{9}^{4} T_{10} T_{11}^{3} T_{12}^{4} T_{13}^{4} T_{14}^{4}+T_{3}^{2} T_{6}-T_{4} T_{5}
\end{array}
$$

and the degree matrix of the $\mathbb{Z}^{9}$-grading is given by

$$
\left[\begin{array}{rrrrrrrrrrrrrr}
-1 & -1 & 0 & 0 & -1 & -1 & 0 & 0 & 0 & 0 & 0 & 0 & 0 & 1 \\
-1 & 0 & 0 & 0 & -1 & -1 & 0 & 0 & 0 & 0 & 0 & 0 & 1 & -1 \\
-1 & 0 & 0 & 0 & -1 & -1 & 0 & 0 & 0 & 0 & 0 & 1 & -1 & 0 \\
0 & 0 & 0 & 0 & -1 & -1 & 0 & 0 & 0 & 0 & 1 & -1 & 0 & 0 \\
1 & 1 & 1 & 2 & 3 & 3 & 0 & 0 & 0 & 0 & 0 & 0 & 0 & 0 \\
0 & -1 & -1 & -1 & -2 & -1 & 0 & 0 & 0 & 1 & 0 & 0 & 0 & 0 \\
0 & -1 & 0 & -1 & 0 & -1 & 0 & 0 & 1 & -1 & 0 & 0 & 0 & 0 \\
0 & 0 & 0 & -1 & 0 & -1 & 0 & 1 & -1 & 0 & 0 & 0 & 0 & 0 \\
0 & 0 & 0 & -1 & 0 & -1 & 1 & -1 & 0 & 0 & 0 & 0 & 0 & 0
\end{array}\right] .
$$

We remark here that the computation of the Cox rings for the minimal resolutions of the surfaces with singularity type $A_{7}$ and $A_{8}$ were not feasible on our systems.

Remark 4.5. Given a CEMDS $X \subseteq Z$ with Cox ring $R$ and a divisor class $w \in$ $\mathrm{Cl}(X)$, consider the graded ring

$$
R(w)=\bigoplus_{m \in \mathbb{Z}_{\geq} 0} R_{m w} \subseteq R .
$$

Given monomial generators $f_{1}, \ldots, f_{s}$ of a Veronese subalgebra $R(d w) \subseteq R(w)$, where $d>0$, one obtains homogeneous equations for $\operatorname{Proj}(R(w))$ by computing the closure of the image of $\bar{X}$ under the toric morphism

$$
\bar{Z} \rightarrow \mathbb{K}^{s}, \quad z \mapsto\left(f_{1}(z), \ldots, f_{s}(z)\right),
$$

where $\bar{Z}$ denotes the ambient toric total coordinate space. If the canonical class of $X$ is known, e.g. if $R$ is a complete intersection Cox ring [26, Prop. 4.15], one obtains this way equations for anticanonical models. For the surfaces $X$ of Theorem 4.1 with a minimal resolution $\widetilde{X}$ of degree 1 , the anticanonical model is always a hypersurface in $\mathbb{P}(1,1,2,3)$. For example, we have the following equations (listed by singularity 
type):

$$
\begin{array}{ll}
2 A_{4}: & T_{1}^{2} T_{2}^{2} T_{3}+T_{1}^{2} T_{2} T_{4}-2 T_{1} T_{2} T_{3}^{2}-T_{1} T_{3} T_{4}+T_{2} T_{3} T_{4}+T_{3}^{3}+T_{4}^{2} \\
D_{8}: & T_{1}^{4} T_{3}-T_{1} T_{2} T_{3}^{2}+T_{3}^{3}+T_{4}^{2} \\
D_{5} A_{3}: & T_{1}^{2} T_{2} T_{4}-T_{1} T_{2} T_{3}^{2}+T_{1} T_{3} T_{4}-T_{3}^{3}-T_{4}^{2} \\
D_{6} 2 A_{1}: & T_{1}^{3} T_{2} T_{3}-3 T_{1}^{2} T_{2}^{2} T_{3}+T_{1}^{2} T_{3}^{2}+3 T_{1} T_{2}^{3} T_{3}-3 T_{1} T_{2} T_{3}^{2}-T_{2}^{4} T_{3}+2 T_{2}^{2} T_{3}^{2}-T_{3}^{3}+T_{4}^{2} \\
E_{6} A_{2}: & T_{1}^{2} T_{2} T_{4}-T_{1} T_{3} T_{4}-T_{3}^{3}-T_{4}^{2} \\
E_{7} A_{1}: & T_{1}^{3} T_{2} T_{3}-T_{1} T_{3} T_{4}-T_{3}^{3}+T_{4}^{2} \\
E_{8}: & T_{1} T_{2}^{5}-T_{2}^{2} T_{3}^{2}-T_{3}^{3}-T_{4}^{2} \\
A_{8}: & T_{1}^{3} T_{4}-T_{2} T_{3} T_{4}+T_{3}^{3}+T_{4}^{2} \\
A_{7} A_{1}: & T_{1}^{2} T_{2} T_{4}-T_{1}^{2} T_{3}^{2}+T_{2} T_{3} T_{4}-T_{3}^{3}+T_{4}^{2}
\end{array}
$$

\section{The lattice ideal method}

We consider the blow up of a given Mori dream space with known Cox ring and develop a method to produce systematically generators for the new Cox ring. The key step is a description of the Cox ring of a blow up as a saturated Rees algebra.

Let $X_{1}$ be a Mori dream space and $\pi: X_{2} \rightarrow X_{1}$ the blow up of an irreducible subvariety $C \subseteq X_{1}$ contained in the smooth locus of $X_{1}$. As before, write $K_{i}:=$ $\mathrm{Cl}\left(X_{i}\right)$ for the divisor class groups and $R_{i}:=\mathcal{R}\left(X_{i}\right)$ for the Cox rings. Then we have the canonical pullback maps

$$
\pi^{*}: K_{1} \rightarrow K_{2},[D] \mapsto\left[\pi^{*} D\right], \quad \pi^{*}: R_{1} \rightarrow R_{2},\left(R_{1}\right)_{[D]} \ni f \mapsto \pi^{*} f \in\left(R_{2}\right)_{\left[\pi^{*} D\right]} .
$$

Moreover, identifying $U:=X_{2} \backslash \pi^{-1}(C)$ with $X_{1} \backslash C$, we obtain canonical push forward maps

$$
\pi_{*}: K_{2} \rightarrow K_{1},[D] \mapsto\left[\pi_{*} D\right], \quad \pi_{*}: R_{2} \rightarrow R_{1},\left(R_{2}\right)_{[D]} \ni f \mapsto f_{\mid U} \in\left(R_{1}\right)_{\left[\pi_{*} D\right]} .
$$

Let $J \subseteq R_{1}$ be the irrelevant ideal, i.e. the vanishing ideal of $\bar{X}_{1} \backslash \widehat{X}_{1}$, and $I \subseteq R_{1}$ the vanishing ideal of $p_{1}^{-1}(C) \subseteq \bar{X}_{1}$ with the characteristic space $p_{1}: \widehat{X}_{1} \rightarrow X_{1}$. We define the saturated Rees algebra to be the subalgebra

$$
R_{1}[I]^{\text {sat }}:=\bigoplus_{d \in \mathbb{Z}}\left(I^{-d}: J^{\infty}\right) t^{d} \subseteq R_{1}\left[t^{ \pm 1}\right], \quad \text { where } I^{k}:=R_{1} \text { for } k \leq 0
$$

Remark 5.1. The usual Rees algebra $R_{1}[I]=\oplus_{\mathbb{Z}} I^{-d} t^{d}$ is a subalgebra of the saturated Rees algebra $R_{1}[I]^{\text {sat }}$. In the above situation, $I \subseteq R_{1}$ is a $K_{1}$-prime ideal and thus we have $I: J^{\infty}=I$. Consequently, $R_{1}[I]^{\text {sat }}$ equals $R_{1}[I]$ if and only if $R_{1}[I]^{\text {sat }}$ is generated in the $\mathbb{Z}$-degrees 0 and \pm 1 . In this case, $R_{1}[I]^{\text {sat }}$ is finitely generated because $R_{1}[I]$ is so.

Note that the saturated Rees algebra $R_{1}[I]^{\text {sat }}$ is naturally graded by $K_{1} \times \mathbb{Z}$ as $R_{1}$ is $K_{1}$-graded and the ideals $I, J$ are homogeneous. Let $E=\pi^{-1}(C)$ denote the exceptional divisor. Then we have a splitting $K_{2}=\pi^{*} K_{1} \times \mathbb{Z} \cdot[E] \cong K_{1} \times \mathbb{Z}$.

Proposition 5.2. In the above situation, we have the following mutually inverse isomorphisms of graded algebras

$$
\begin{aligned}
R_{2} & \longleftrightarrow R_{1}[I]^{\mathrm{sat}}, \\
\left(R_{2}\right)_{\left[\pi^{*} D\right]+d[E]} \ni f & \mapsto \pi_{*} f \cdot t^{d} \in\left(R_{1}[I]^{\mathrm{sat}}\right)_{([D], d)}, \\
\left(R_{2}\right)_{\left[\pi^{*} D\right]+d[E]} \ni \pi^{*} f \cdot 1_{E}^{d} & \longleftrightarrow \quad f \cdot t^{d} \in\left(R_{1}[I]^{\mathrm{sat}}\right)_{([D], d)} .
\end{aligned}
$$

Lemma 5.3. In the above situation, consider the characteristic spaces $p_{i}: \widehat{X}_{i} \rightarrow X_{i}$ and let $\mathcal{I}_{C}, \mathcal{I}_{\widehat{C}}, \mathcal{I}_{E}, \mathcal{I}_{\widehat{E}}$ be the ideal sheaves of $C, \widehat{C}=p_{1}^{-1}(C), E, \widehat{E}=p_{2}^{-1}(E)$ on $X_{1}, \widehat{X}_{1}, X_{2}, \widehat{X}_{2}$ respectively. Then, for any $m>0$, we have

$$
p_{1}^{*}\left(\mathcal{I}_{C}^{m}\right)=\mathcal{I}_{\widehat{C}}^{m}, \quad p_{2}^{*}\left(\mathcal{I}_{E}^{m}\right)=\mathcal{I}_{\widehat{E}}^{m}, \quad \pi^{*}\left(\mathcal{I}_{C}^{m}\right)=\mathcal{I}_{E}^{m} .
$$


Moreover, with the vanishing ideals $I \subseteq R_{1}$ of the closure of $\widehat{C}$ and $J \subseteq R_{1}$ of Spec $R_{1} \backslash \widehat{X}_{1}$, we have $\Gamma\left(\widehat{X}_{1}, \mathcal{I}_{\widehat{C}}^{m}\right)=I^{m}: J^{\infty}$ for any $m>0$.

Proof. For the first equality, we use that $C$ is contained in the smooth locus of $X_{1}$. This implies that $p_{1}$ has no multiple fibers near $C$ and the claim follows. The second equality is obtained by the same reasoning. The third one is a standard fact on blowing up [24, Prop. 8.1.7 and Cor. 8.1.8]. The last statement follows from the fact that $\widehat{X}_{1}$ is quasiaffine.

Proof of Proposition 5.2. We only have to prove that the maps are well defined. For the map from $R_{2}$ to $R_{1}[I]^{\text {sat }}$ consider $f \in\left(R_{2}\right)_{\left(\left[\pi^{*} D\right], d\right)}$. We have to show that for $d<0$, the push forward $\pi_{*} f$ belongs to $I^{-d}: J^{\infty}$. Note that $\pi_{*} f=\pi_{*} f^{\prime}$ holds with $f^{\prime}:=f \cdot 1_{E}^{-d} \in\left(R_{2}\right)_{\left[\pi^{*} D\right]}$. Pushing $f^{\prime}$ locally to $X_{2}$, then to $X_{1}$ and finally lifting it to $\widehat{X}_{1}$, we see using Lemma 5.3 that $\pi_{*} f^{\prime}$ is a global section of the $-d$-th power of the ideal sheaf of $p_{1}^{-1}(C) \subseteq \widehat{X}_{1}$. This gives $\pi_{*} f^{\prime} \in I^{-d}: J^{\infty} \subseteq R_{1}$.

For the map from $R_{1}[I]^{\text {sat }}$ to $R_{2}$, consider $f \cdot t^{d} \in\left(R_{1}[I]^{\text {sat }}\right)_{([D], d)}$, where $d<0$. We need that $1_{E}^{-d}$ divides $\pi^{*} f$ in $R_{2}$. By definition, there exist an $h \in J$ and a $k \geq 0$ such that $h \notin I$ and $f h^{k} \in I^{-d}$. Then we have $\left(\pi^{*} f\right)\left(\pi^{*} h\right)^{k} \in\left(\pi^{*} I\right)^{-d}$. Using $\left\langle\pi^{*} I\right\rangle=\left\langle 1_{E}\right\rangle$ and the fact that $1_{E}$ is a $K_{2}$-prime not dividing any power of $\pi^{*} h$ we see that $1_{E}^{-d}$ divides $\pi^{*} f$.

For the computation of the Cox ring $R_{2}$, we work in the notation of Setting 2.5 in particular $X_{1}$ comes as a CEMDS $X_{1} \subseteq Z_{1}$. As before, $C \subseteq X_{1}$ is an irreducible subvariety contained in the smooth locus of $X_{1}$ and $\widehat{C} \subseteq \widehat{X}_{1}$ denotes its inverse image with respect to $p_{1}: \widehat{X}_{1} \rightarrow X_{1}$. The idea is to stretch the given embedding $X_{1} \subseteq Z_{1}$ by suitable generators of the vanishing ideal $I \subseteq R_{1}$ of $\widehat{C} \subseteq \bar{X}_{1}$ and then perform an ambient modification.

Algorithm 5.4 (BlowUpCEMDS). Input: a CEMDS $\left(P_{1}, \Sigma_{1}, G_{1}\right)$, a $K_{1}$-prime ideal $I=\left\langle f_{1}, \ldots, f_{l}\right\rangle \subseteq R_{1}$ with pairwise non-associated $K_{1}$-primes $f_{i} \in R_{1}$ defining an irreducible subvariety $C \subseteq X_{1}$ inside the smooth locus and coprime positive integers $d_{1}, \ldots, d_{l}$ with $f_{i} \in I^{\bar{d}_{i}}: J^{\infty}$.

- Compute the stretched CEMDS $\left(P_{1}^{\prime}, \Sigma_{1}^{\prime}, G_{1}^{\prime}\right)$ by applying Algorithm 3.1 to $\left(P_{1}, \Sigma_{1}, G_{1}\right)$ and $\left(f_{1}, \ldots, f_{l}\right)$.

- Define a multiplicity vector $v \in \mathbb{Z}^{r_{1}+l}$ by $v_{i}:=0$ for $1 \leq i \leq r_{1}$ and $v_{i}:=d_{i-r_{1}}$ for $r_{1}+1 \leq i \leq r_{1}+l$.

- Determine the stellar subdivision $\Sigma_{2} \rightarrow \Sigma_{1}^{\prime}$ of the fan $\Sigma_{1}^{\prime}$ along the ray through $P_{1}^{\prime} \cdot v$. Set $P_{2}:=\left[P_{1}^{\prime}, P_{1}^{\prime} \cdot v\right]$.

- Compute $\left(P_{2}, \Sigma_{2}, G_{2}\right)$ by applying Algorithm 3.6 to $\left(P_{1}^{\prime}, \Sigma_{1}^{\prime}, G_{1}^{\prime}\right)$ and the pair $\left(P_{2}, \Sigma_{2}\right)$.

- Let $T^{\nu}$ be the product over all $T_{i}$ with $C \nsubseteq D_{i}$ where $D_{i} \subseteq X_{1}$ is the divisor corresponding to $T_{i}$. Test whether $\left.\operatorname{dim}\left(I_{2}+\left\langle T_{r_{2}}\right\rangle\right)\right\rangle \operatorname{dim}\left(I_{2}+\left\langle T_{r_{2}}, T^{\nu}\right\rangle\right)$.

- Set $\left(P_{2}^{\prime}, \Sigma_{2}^{\prime}, G_{2}^{\prime}\right):=\left(P_{2}, \Sigma_{2}, G_{2}\right)$. Eliminate all fake relations by applying Algorithm 3.2. Call the output $\left(P_{2}, \Sigma_{2}, G_{2}\right)$.

Output: $\left(P_{2}, \Sigma_{2}, G_{2}\right)$. If the verification in the next to last step was positive, then $\left(P_{2}, \Sigma_{2}, G_{2}\right)$ is a CEMDS describing the blow up $X_{2}$ of $X_{1}$ along $C$. In particular then the $K_{2}$-graded algebra $R_{2}$ is the Cox ring of $X_{2}$.

Proof. Consider the $K_{2}$-graded ring $R_{2}=\mathbb{K}\left[T_{1}, \ldots, T_{r_{2}}\right] / I_{2}$ associated to the output $\left(P_{2}, \Sigma_{2}, G_{2}\right)$. The first step is to show that $R_{2}$ is normal; then $\left(P_{2}, \Sigma_{2}, G_{2}\right)$ is a CEMDS and $R_{2}$ is the Cox ring of the output variety $X_{2}$. In a second step we show that $X_{2}$ equals the blow up of $X_{1}$ along $C$.

Consider the output $\left(P_{2}, \Sigma_{2}, G_{2}\right)$ of the fourth item, i.e. the situation before eliminating fake relations. The variables $T_{r_{1}+1}, \ldots, T_{r_{2}-1}$ correspond to $f_{1}, \ldots, f_{l}$ 
and $T_{r_{2}}$ to the exceptional divisor. Observe that we have a canonical $K_{2}$-graded homomorphism $R_{2} \rightarrow R_{1}[I]^{\text {sat }}$ induced by

$$
\mathbb{K}\left[T_{1}, \ldots, T_{r_{2}}\right] \rightarrow R_{1}[I]^{\mathrm{sat}}, \quad T_{i} \mapsto \begin{cases}T_{i}, & 1 \leq i \leq r_{1}, \\ f_{i-r_{1}} t^{-v_{i}}, & r_{1}<i<r_{2}, \\ t, & i=r_{2} .\end{cases}
$$

Indeed, because $C$ is contained in the smooth locus of $X_{1}$, the cone generated by the last $l$ columns of $P_{1}^{\prime}$ is regular and, because in addition $d_{1}, \ldots, d_{l}$ are coprime, the vector $P_{1}^{\prime} \cdot v$ is primitive. Thus, the ideal $I_{2}$ of $X_{2}$ is the saturation with respect to $T_{r_{2}}$ of

$$
I_{1}+\left\langle T_{i} T_{r_{2}}^{v_{i}}-f_{i-r_{1}} ; r_{1}<i<r_{2}\right\rangle \subseteq \mathbb{K}\left[T_{1}, \ldots, T_{r_{2}}\right] .
$$

Consequently, the above assignment induces a homomorphism $R_{2} \rightarrow R_{1}[I]^{\text {sat }}$. This homomorphism induces an isomorphism of the $K_{2}$-graded localizations

$$
\left(R_{2}\right)_{T_{r_{2}}}=\bigoplus_{d \in \mathbb{Z}} R_{1} T_{r_{2}}^{d} \cong \bigoplus_{d \in \mathbb{Z}} R_{1} t^{d}=\left(R_{1}[I]^{\mathrm{sat}}\right)_{t}
$$

and hence is in particular injective. As the image $\varphi\left(R_{2}\right)$ contains generators $t=$ $\varphi\left(T_{r_{2}}\right)$ and $\varphi\left(T_{r_{1}+i} T_{r_{2}}^{v_{i}-1}\right)=f_{i} t^{-1}$ for the Rees algebra $R_{1}[I]$, we obtain

$$
R_{1}[I] \subseteq \varphi\left(R_{2}\right) \subseteq R_{1}[I]^{\text {sat }} .
$$

We show that $\varphi\left(R_{2}\right)$ equals $R_{1}[I]^{\text {sat }}$. Otherwise, the algebras must be different in some degree, i.e. we can choose $n \in \mathbb{Z}_{\geq 1}$ minimal such that there is $f t^{-n} \in$ $\left(R_{1}[I]^{\text {sat }}\right)_{-n} \backslash \varphi\left(R_{2}\right)_{-n}$. The minimality implies $f t^{-n+1} \in\langle t\rangle_{R_{1}[I]^{\text {sat }}} \cap \varphi\left(R_{2}\right)$ with $f t^{-n+1} \notin\langle t\rangle_{\varphi\left(R_{2}\right)}$. In particular, $\langle t\rangle_{\varphi\left(R_{2}\right)}$ is properly contained in $\langle t\rangle_{R_{1}[I]^{\mathrm{sat}}} \cap \varphi\left(R_{2}\right)$.

Define $T^{\nu}$ as the product over all $T_{i}$ such that $C \nsubseteq D_{i}$ where $D_{i} \subseteq X_{1}$ is the divisor corresponding to $T_{i}$. Note that $T^{\nu} \in J \subseteq R_{1}$. In the localized algebras

$$
R_{1}[I]_{T^{\nu}}=\varphi\left(R_{2}\right)_{T^{\nu}}=\left(R_{1}[I]^{\mathrm{sat}}\right)_{T^{\nu}}
$$

the ideal $\langle t\rangle_{\varphi\left(R_{2}\right)}$ and the $K_{2}$-prime ideal $\langle t\rangle_{R_{1}[I]^{\text {sat }}} \cap \varphi\left(R_{2}\right)$ coincide, i.e. $t$ is $K_{2^{-}}$ prime in $\varphi\left(R_{2}\right)_{T^{\nu}}$. By the dimension test we know that $t$ and $T^{\nu}$ are coprime in $\varphi\left(R_{2}\right)$. Consequently, $t$ is $K_{2}$-prime in $\varphi\left(R_{2}\right)$. Therefore, $\langle t\rangle_{R_{1}[I]^{\mathrm{sat}}} \cap \varphi\left(R_{2}\right)=$ $\langle t\rangle_{\varphi\left(R_{2}\right)}$ in $\varphi\left(R_{2}\right)$, a contradiction. We conclude $R_{2} \cong R_{1}[I]^{\text {sat }}$.

By Proposition 5.2, $R_{1}[I]^{\text {sat }} \cong R_{2}$ is the Cox ring of the blow up $X_{2}^{\prime}$ of $X_{1}$ at $C$. In particular, $R_{2} \cong R_{2}^{\prime}$ is normal. We may now apply Algorithm 3.2 . Note that there is no need to use the verify option as the variables $T_{1}, \ldots, T_{r_{2}} \in R_{2}$ are $K_{2}$-prime and the generators surviving the elimination process are $K_{2}$-prime as well. As for any Cox ring, the $K_{2}$-grading is almost-free.

We show that $X_{2} \cong X_{2}^{\prime}$ holds. Let $\lambda \subseteq \operatorname{Mov}\left(R_{2}\right)$ be the chamber representing $X_{1}$. Then $\lambda$ is of codimension one in $\mathbb{Q} \otimes K_{2}$ and lies on the boundary of $\operatorname{Mov}\left(R_{2}\right)$. Since there are the contraction morphisms $X_{2} \rightarrow X_{1}$ and $X_{2}^{\prime} \rightarrow X_{1}$, the chambers $\lambda_{2}, \lambda_{2}^{\prime}$ corresponding to $X_{2}, X_{2}^{\prime}$ both have $\lambda$ as a face. We conclude $\lambda_{2}=\lambda_{2}^{\prime}$ and thus $X_{2} \cong X_{2}^{\prime}$.

An important special case of Algorithm 5.4 is blowing up a smooth point. The point $x_{1} \in X_{1}$ can be given in Cox coordinates, i.e. as a point $z \in \widehat{X}_{1} \subseteq \mathbb{K}^{r_{1}}$ with $x_{1}=p_{1}(z)$. The steps are as follows.

Remark 5.5 (Blow up of a point). Let $X_{1}=\left(P_{1}, \Sigma_{1}, G_{1}\right)$ be a CEMDS and $x \in X_{1}$ a smooth point given in Cox coordinates $z \in \mathbb{K}^{r_{1}}$. Let $i_{1}, \ldots, i_{k}$ be the indices with $z_{i_{j}} \neq 0$ and $\nu_{1}, \ldots, \nu_{s} \in \mathbb{Z}^{r}$ a lattice basis for $\operatorname{im}\left(P_{1}^{*}\right) \cap \operatorname{lin}\left(e_{i_{1}}, \ldots, e_{i_{k}}\right)$. The associated ideal to $P_{1}$ and $z$ is

$$
\begin{aligned}
I\left(P_{1}, z\right):= & \left\langle z^{-\nu_{1}^{+}} T^{\nu_{1}^{+}}-z^{-\nu_{1}^{-}} T^{\nu_{1}^{-}}, \ldots, z^{-\nu_{s}^{+}} T^{\nu_{s}^{+}}-z^{-\nu_{s}^{-}} T^{\nu_{s}^{-}}\right\rangle:\left(T_{1} \cdots T_{r}\right)^{\infty} \\
& +\left\langle T_{j} ; z_{j}=0\right\rangle \subseteq \mathbb{K}\left[T_{1}, \ldots, T_{r}\right],
\end{aligned}
$$


where $\nu_{i}=\nu_{i}^{+}+\nu_{i}^{-}$is the unique representation as a sum of a nonnegative and a nonpositive vector having disjoint supports. The ideal is generated by variables and binomials; for general $z$ it is a lattice ideal see 39 . Then $I\left(P_{1}, z\right) \subseteq R_{1}$ is the vanishing ideal of the orbit closure $\overline{H_{1} \cdot z}$ in $\bar{X}_{1}$. Let $\left(f_{1}, \ldots, f_{l}\right)$ be a list of pairwise non-associated $K_{1}$-prime generators for $I\left(P_{1}, z_{1}\right) \subseteq R_{1}$ and $d_{1}, \ldots, d_{l} \in \mathbb{Z}_{\geq 1}$. Then the Cox ring of the blow up of $X_{1}$ in $x$ can be computed with Algorithm 5.4 with input $\left(f_{1}, \ldots, f_{l}\right)$ and $\left(d_{1}, \ldots, d_{l}\right)$.

The following algorithm produces systematically generators and their multiplicities $d_{i} \in \mathbb{Z}_{\geq 1}$ of the Cox ring of a blow up of a Mori dream space in the sense that adds step by step generator sets for the positive Rees algebra components.

Algorithm 5.6 (BlowUpCEMDS2). Input: a CEMDS $\left(P_{1}, \Sigma_{1}, G_{1}\right)$, a $K_{1}$-prime ideal $I$ defining an irreducible subvariety $C \subseteq X_{1}$ inside the smooth locus.

- Let $F$ and $D$ be empty lists.

- For each $k=1,2, \ldots \in \mathbb{Z}_{\geq 1}$ do

- compute a set $G_{k}$ of generators for $A_{k}:=I^{k}: J^{\infty} \subseteq R_{1}$. Let $f_{k 1}, \ldots, f_{k l_{i}}$ be a maximal subset of pairwise non-associated elements of $G_{k}$ with

$$
f_{k j} \notin A_{1} A_{k-1}+\ldots+A_{\left\lfloor\frac{k}{2}\right\rfloor} A_{\left\lceil\frac{k}{2}\right\rceil} \quad \text { if } k>1 .
$$

- Determine integers $d_{k 1}, \ldots, d_{k l_{i}} \in \mathbb{Z}_{\geq k}$ such that $f_{k j} \in A_{d_{k i}} \backslash A_{d_{k i}+1}$.

- Add the elements of $f_{k 1}, \ldots, f_{k l_{i}}$ to $F$ that are not associated to any other element of $F$. Add the respective integers among $d_{k 1}, \ldots, d_{k l_{i}}$ to $D$.

- Run Algorithm 5.4 with input $\left(P_{1}, \Sigma_{1}, G_{1}\right), F$ and $D$.

- If Algorithm 5.4 terminated with $\left(P_{2}, \Sigma_{2}, G_{2}\right)$ and positive verification, return $\left(P_{2}, \Sigma_{2}, G_{2}\right)$.

Output (if provided): the algorithm terminates if and only if $X_{2}$ is a Mori dream space. In this case, the CEMDS $\left(P_{2}, \Sigma_{2}, G_{2}\right)$ describes the blow up $X_{2}$ of $X_{1}$ along $C$. In particular then the $K_{2}$-graded algebra $R_{2}$ is the Cox ring of $X_{2}$.

Proof. Note that each $f_{k i}$ is a $K_{1}$-prime element. Otherwise, $f_{k i}=f_{1} f_{2}$ with $K_{1}$ homogeneous elements $f_{i} \in R_{1}$. As $I$ is $K_{1}$-prime, $f_{1}$ or $f_{2}$ lies in $A_{k^{\prime}}$ with $k^{\prime}<k$, i.e. $f_{k i} \in A_{k^{\prime}}$. This contradicts the choice of $f_{k i}$.

By Proposition 5.2, the Cox ring $R_{2}$ of the blow up is isomorphic to the saturated Rees algebra $R_{1}[I]^{\text {sat }}$. After the $k$-th step, $\left(F, T_{1}, \ldots, T_{r_{1}}, t\right)$ are generators for a subalgebra $B_{k} \subseteq R_{1}[I]^{\text {sat }}$ such that

$$
\mathbb{K}\left[\{t\} \cup R_{1} \cup A_{1} t^{-1} \cup \ldots \cup A_{k} t^{-k}\right] \subseteq B_{k} \subseteq \bigoplus_{k \in \mathbb{Z}} A_{k} t^{-k}=R_{1}[I]^{\mathrm{sat}} .
$$

If the algorithm stops, by the correctness of Algorithm 5.4, the output then is a CEMDS describing the blow $X_{2}$ with Cox ring $R_{2}$. Vice versa, if $X_{2}$ has finitely generated Cox ring, there is $k_{0} \geq 1$ with $R_{1}[I]^{\text {sat }}=B_{k_{0}}$. Then Algorithm 5.4 is called with $K_{1}$-prime non-associated generators for $\mathcal{R}\left(X_{2}\right) \cong R_{1}[I]^{\text {sat }}$ and thus terminates with positive verification.

Example 5.7. We compute the Cox ring of the blow up of the weighted projective plane $X_{1}:=\mathbb{P}_{3,4,5}$ at the general point with Cox coordinates $z_{1}:=(1,1,1) \in \mathbb{K}^{3}$ by the steps of Algorithm 5.6. The lattice ideal of $z_{1}$ with respect to $P_{1}$ is

$$
I\left(P_{1}, z_{1}\right)=\left\langle T_{2}^{2}-T_{1} T_{3}, T_{1}^{2} T_{2}-T_{3}^{2}, T_{1}^{3}-T_{2} T_{3}\right\rangle, \quad P_{1}:=\left[\begin{array}{rrr}
1 & -2 & 1 \\
-2 & -1 & 2
\end{array}\right] .
$$

An application of Algorithm 5.4 with the three generators $\left(f_{1}, f_{2}, f_{3}\right)$ of $I:=$ $I\left(P_{1}, z_{1}\right)$ and all $d_{i}:=1$ fails. However, adding the additional generator

$$
f_{4}:=T_{1}^{5}-3 T_{1}^{2} T_{2} T_{3}+T_{1} T_{2}^{3}+T_{3}^{3} \in I^{2}: J^{\infty}
$$


with $d_{4}:=2$ to the input, Algorithm 5.4 returns the $\mathrm{Cl}\left(X_{2}\right)=\mathbb{Z}^{2}$-graded Cox ring $R_{2}=\mathcal{R}\left(X_{2}\right)$ of the blow up $X_{2}$ of $X_{1}$ in $\left[z_{1}\right]$. All verifications are positive. The ring is given as $R_{2}=\mathbb{K}\left[T_{1}, \ldots, T_{8}\right] / I_{2}$ with generators for $I_{2}$ and the degree matrix being

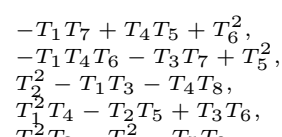

In Algorithm 5.4, the saturation computation may become infeasible. In this case, the following variant can be used to obtain at least finite generation.

Algorithm 5.8 (Finite generation). Input: a CEMDS $\left(P_{1}, \Sigma_{1}, G_{1}\right)$, a $K_{1}$-prime ideal $I=\left\langle f_{1}, \ldots, f_{l}\right\rangle \subseteq R_{1}$ with pairwise non-associated $K_{1}$-primes $f_{i}$ defining an irreducible subvariety $C \subseteq X_{1}$ inside the smooth locus and coprime positive integers $d_{1}, \ldots, d_{l}$ with $f_{i} \in I^{d_{i}}: J^{\infty}$.

- Compute the stretched CEMDS $\left(P_{1}^{\prime}, \Sigma_{1}^{\prime}, G_{1}^{\prime}\right)$ by applying Algorithm 3.1 to $\left(P_{1}, \Sigma_{1}, G_{1}\right)$ and $\left(f_{1}, \ldots, f_{l}\right)$.

- Define a multiplicity vector $v \in \mathbb{Z}^{r_{1}+l}$ by $v_{i}:=0$ if $1 \leq i \leq r_{1}$ and $v_{i}:=d_{i-r_{1}}$ for $r_{1}+1 \leq i \leq r_{1}+l$.

- Determine the stellar subdivision $\Sigma_{2} \rightarrow \Sigma_{1}^{\prime}$ of the fan $\Sigma_{1}^{\prime}$ along the ray through $P_{1}^{\prime} \cdot v$. Set $P_{2}:=\left[P_{1}^{\prime}, P_{1}^{\prime} \cdot v\right]$.

- Compute $G_{2}^{\prime}:=\left(h_{1}, \ldots, h_{s}\right)$, where $h_{i}=p_{2}^{\sharp}\left(p_{1}^{\prime}\right)_{\sharp}\left(g_{i}\right)$ and $G_{1}^{\prime}=\left(g_{1}, \ldots, g_{s}\right)$.

- Choose a system of generators $G_{2}$ of an ideal $I_{2} \subseteq \mathbb{K}\left[T_{1}, \ldots, T_{r_{2}}\right]$ with $\left\langle G_{2}^{\prime}\right\rangle:\left(T_{1} \cdots T_{r_{2}}\right)^{\infty} \supseteq I_{2} \supseteq\left\langle G_{2}^{\prime}\right\rangle$.

- Check if $\operatorname{dim}\left(I_{2}\right)-\operatorname{dim}\left(I_{2}+\left\langle T_{i}, T_{j}\right\rangle\right) \geq 2$ for all $i \neq j$.

- Check if $T_{r_{2}}$ is prime in $\mathbb{K}\left[T_{j}^{ \pm 1} ; j \neq r_{2}\right]\left[T_{r_{2}}\right] / I_{2}$.

Output: $\left(P_{2}, \Sigma_{2}, G_{2}\right)$. The ES $\left(P_{2}, \Sigma_{2}, G_{2}\right)$ describes the blow up $X_{2}$ of $X_{1}$ along $C$. If all verifications in the last steps were positive, the Cox ring $\mathcal{R}\left(X_{2}\right)$ is finitely generated and is given by the $H_{2}$-equivariant normalization of $\mathbb{K}\left[T_{1}, \ldots, T_{r_{2}}\right] / I_{2}$ : $\left(T_{1} \cdots T_{r_{2}}\right)^{\infty}$.

Proof. By the last verification, the exceptional divisor $D_{r_{2}} \subseteq X_{2}$ inherits a local defining equation from the toric ambient variety $Z_{2}$. Thus, the ambient modification is neat in the sense of [26, Def. 5.4]. By [26, Prop. 5.5], $X_{2} \subseteq Z_{2}$ is a neat embedding. In turn, the dimension checks enable us to use [26, Cor. 2.7], which completes the proof.

\section{Smooth Rational SURfaces}

We consider smooth rational surfaces $X$ of Picard number $\varrho(X) \leq 6$. Using Algorithm 5.4, we show that they are all Mori dream surfaces and we compute their Cox rings. Recall that every smooth rational surface $X$ of Picard number $\varrho(X)=k$ can be obtained by blowing up the projective plane $\mathbb{P}_{2}$ at $k-1$ points or a Hirzebruch surface $\mathbb{F}_{a}$ at $k-2$ points, where, in both cases, some points may be infinitely near, i.e. one also performs iterated blow ups. Whereas blow ups of the projective plane $\mathbb{P}_{2}$ can be done in a purely computational manner, the treatment of the (infinitely many) Hirzebruch surfaces $\mathbb{F}_{a}$ requires also theoretical work due to their parameter $a \in \mathbb{Z}_{\geq 1}$.

In the following statement, we concentrate on those surfaces $X$ that do not admit a (non-trivial) $\mathbb{K}^{*}$-action; for the full list of Cox rings in the case $\varrho(X) \leq$ 5 , we refer to 33 . Note that the rational $\mathbb{K}^{*}$-surfaces as well as the toric ones admit a combinatorial description which opens a direct approach to their Cox rings, see [27, 29. We denote the iterated blow up of a point $x$ by sequences of $g_{i}$ and $s_{i}$ indicating general and special points on the $i$-th exceptional divisor over $x$. For example, $s_{3} g_{2} g_{1}$ indicates a fourfold blow up of a point with a general point $g_{1}$ on 
the first exceptional divisor, a general point $g_{2}$ on the second and a special point $s_{3}$ on the third. The special points will be precisely defined in each case.

Theorem 6.1. Let $X$ be a smooth rational surface with Picard number $\varrho(X) \leq 6$. Then $X$ is a Mori dream space. If $\varrho(X) \leq 5$, then either $X$ admits a $\mathbb{K}^{*}$-action or is isomorphic to $\bar{M}_{0,5}$, the blow up of $\mathbb{P}_{2}$ in four general points. If $\varrho(X)=6$, then $X$ admits a $\mathbb{K}^{*}$-action or its Cox ring is isomorphic to exactly one of the following, where $a \in \mathbb{Z}_{\geq 3}$.

(i) The $\mathbb{Z}^{6}$-graded ring $\mathbb{K}\left[T_{1}, \ldots, T_{10}\right] / I$, where generators for $I$ and the degree matrix are given as

$$
\begin{gathered}
T_{3}^{2} T_{4}-T_{1} T_{2}-T_{6} T_{7} T_{8} T_{10}, T_{1} T_{2}^{2} T_{3} T_{4} T_{5}-T_{6}^{2} T_{7}-T_{9} T_{10} \\
{\left[\begin{array}{rrrrrrrrrrr}
1 & 0 & 0 & 1 & 0 & 0 & 2 & 0 & 3 & -1 \\
0 & 1 & 0 & 1 & 0 & 0 & 3 & 0 & 5 & -2 \\
0 & 0 & 1 & -2 & 0 & 0 & -1 & 0 & -2 & 1 \\
0 & 0 & 0 & 0 & 1 & 0 & 1 & 0 & 2 & -1 \\
0 & 0 & 0 & 0 & 0 & 1 & -2 & 0 & -1 & 1 \\
0 & 0 & 0 & 0 & 0 & 0 & 0 & 1 & 1 & -1
\end{array}\right]}
\end{gathered}
$$

In this case, $X$ is a 5 -fold blow up of $\mathbb{P}_{2}$ in a point of type $g_{4} s_{3} g_{2} g_{2}$, where $s_{3}$ is the intersection point of the 3rd and the 2nd exceptional divisor.

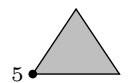

(ii) The $\mathbb{Z}^{6}$-graded ring $\mathbb{K}\left[T_{1}, \ldots, T_{10}\right] / I$, where generators for $I$ and the degree matrix are given as

$$
\begin{gathered}
T_{3} T_{5} T_{8}-T_{2} T_{6}-T_{9} T_{10}, T_{1} T_{5}+T_{7} T_{8}-T_{2} T_{6}^{2} T_{4} T_{10} \\
{\left[\begin{array}{rlllllrrrr}
1 & 0 & 0 & 0 & 0 & 0 & 1 & 0 & -1 & 1 \\
0 & 1 & 0 & 0 & 0 & 0 & -1 & 1 & 2 & -1 \\
0 & 0 & 1 & 0 & 0 & 0 & 1 & -1 & 0 & 0 \\
0 & 0 & 0 & 1 & 0 & 0 & 0 & 0 & 1 & -1 \\
0 & 0 & 0 & 0 & 1 & 0 & 2 & -1 & -1 & 1 \\
0 & 0 & 0 & 0 & 0 & 1 & -1 & 1 & 3 & -2
\end{array}\right]}
\end{gathered}
$$

In this case, $X$ is a 3 -fold blow up of $\mathbb{P}_{2}$ in $[1,0,0]$ of type $g_{1} g_{1}$, and single blow ups in $[0,1,0]$ and $[1,1,0]$.

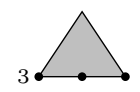

(iii) The $\mathbb{Z}^{6}$-graded ring $\mathbb{K}\left[T_{1}, \ldots, T_{11}\right] / I$, where generators for $I$ and the degree matrix are given as

$$
\begin{aligned}
& T_{3}^{2} T_{4} T_{5}^{2} T_{8}-T_{2} T_{7}-T_{11} T_{10}, \\
& T_{1} T_{5}+T_{7} T_{8}-T_{2} T_{4} T_{6}^{2} T_{11}^{2}, \\
& T_{3}^{2} T_{4}^{2} T_{5} T_{8} T_{2} T_{6}^{2} T_{11}-T_{7} T_{9} T_{4} T_{6}^{2} T_{11}-T_{5} T_{10}+T_{8} T_{10},
\end{aligned}
$$

In this case, $X$ is the 3 -fold blow up of $\mathbb{P}_{2}$ in $[1,0,0]$ of type $g_{2} g_{1}$ and single blow ups in $[0,1,0]$ and $[1,1,0]$.

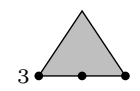

(iv) The $\mathbb{Z}^{6}$-graded ring $\mathbb{K}\left[T_{1}, \ldots, T_{10}\right] / I$, where generators for $I$ and the degree matrix are given as

$$
\begin{gathered}
T_{1} T_{5}+T_{7} T_{8}-T_{2} T_{4} T_{6} T_{10}, T_{3} T_{5} T_{7} T_{8}^{2}-T_{2}^{2} T_{4}-T_{9} T_{10} \\
{\left[\begin{array}{rrrrrrrrrr}
1 & 0 & 0 & 0 & 0 & 0 & 2 & -1 & -1 & 1 \\
0 & 1 & 0 & 0 & 0 & 0 & -2 & 2 & 3 & -1 \\
0 & 0 & 1 & 0 & 0 & 0 & 1 & -1 & 0 & 0 \\
0 & 0 & 0 & 1 & 0 & 0 & -1 & 1 & 2 & -1 \\
0 & 0 & 0 & 0 & 1 & 0 & 3 & -2 & -1 & 1 \\
0 & 0 & 0 & 0 & 0 & 1 & 0 & 0 & 1 & -1
\end{array}\right]}
\end{gathered}
$$


In this case, $X$ is the 3 -fold blow up of $\mathbb{P}_{2}$ in $[1,0,0]$ of type $g_{1} s_{1}$ with the intersection point $s_{1}$ of the 1 st exceptional divisor and the transform of $V\left(T_{3}\right) \subseteq \mathbb{P}_{2}$ and single blow ups in $[0,1,0]$ and $[1,1,0]$.

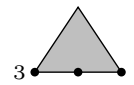

(v) The $\mathbb{Z}^{6}$-graded ring $\mathbb{K}\left[T_{1}, \ldots, T_{13}\right] / I$, where generators for $I$ and the degree matrix are given as

$$
\begin{aligned}
& T_{1} T_{11}-T_{4} T_{3} T_{9}-T_{8} T_{12}, \quad T_{1} T_{7}-T_{2} T_{8}+T_{3} T_{9} T_{13}, \\
& T_{2} T_{6}+T_{7} T_{10}-T_{3} T_{5} T_{13}, \quad T_{1} T_{6}+T_{8} T_{10}-T_{3} T_{4} T_{13}, \\
& T_{2} T_{11}-\lambda T_{5} T_{3} T_{9}-T_{7} T_{12}, \quad(\lambda-1) T_{1} T_{5}-T_{10} T_{9}-T_{12} T_{13}, \\
& \begin{array}{ll}
(\lambda-1) T_{5} T_{8}+T_{6} T_{9}-T_{11} T_{13}, & T_{10} T_{11}-(\lambda-1) T_{4} T_{3} T_{5}+T_{6} T_{12} \\
(\lambda-1) T_{4} T_{7}+\lambda T_{6} T_{9}-T_{11} T_{13}, & (\lambda-1) T_{2} T_{4}-\lambda T_{10} T_{9}-T_{12} T_{13} .
\end{array} \\
& {\left[\begin{array}{rrrrrrrrrrrrr}
1 & 0 & 0 & 0 & -1 & 0 & 0 & 1 & 0 & 0 & -1 & -1 & 1 \\
0 & 1 & 0 & 0 & 1 & 0 & 0 & -1 & 0 & 1 & 0 & 1 & 0 \\
0 & 0 & 1 & 0 & 0 & 0 & 0 & 0 & 0 & 0 & 1 & 1 & -1 \\
0 & 0 & 0 & 1 & 1 & 0 & 0 & 0 & 1 & 0 & 2 & 2 & -1 \\
0 & 0 & 0 & 0 & 0 & 1 & 0 & 0 & -1 & 1 & -1 & -1 & 1 \\
0 & 0 & 0 & 0 & 0 & 0 & 1 & 1 & 1 & -1 & 1 & 0 & 0
\end{array}\right]}
\end{aligned}
$$

In this case, $X$ is the the blow up of $\mathbb{P}_{2}$ in $[1,0,0],[0,1,0],[0,0,1],[1,1,1]$ and $[1, \lambda, 0]$ where $\lambda \in \mathbb{K}^{*} \backslash\{1\}$.

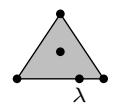

(vi) The $\mathbb{Z}^{6}$-graded ring $\mathbb{K}\left[T_{1}, \ldots, T_{16}\right] / I$, where generators for $I$ and the degree matrix are given as

$$
\begin{array}{ll}
T_{6} T_{12}+\lambda T_{7} T_{14}-T_{8} T_{13}, & T_{5} T_{12}-\mu T_{7} T_{15}-T_{9} T_{13}, \\
T_{4} T_{13}-\lambda T_{5} T_{14}-\mu T_{6} T_{15}, & T_{4} T_{12}-\mu T_{8} T_{15}-\lambda T_{9} T_{14}, \\
T_{3} T_{11}+T_{7} T_{14}-T_{8} T_{13}, & T_{1} T_{13}-T_{2} T_{14}-T_{3} T_{15}, \\
T_{1} T_{11}-T_{8} T_{15}-T_{9} T_{14}, & T_{2} T_{11}-T_{7} T_{15}-T_{9} T_{13}, \\
(\lambda-\mu) T_{3} T_{5}+\mu T_{7} T_{10}-T_{13} T_{16}, & (-\lambda+1) T_{5} T_{14}+(-\mu+1) T_{6} T_{15}+T_{10} T_{11}, \\
(\lambda-1) T_{5} T_{8}+(-\mu+1) T_{6} T_{9}-T_{11} T_{16}, & (\lambda-1) T_{4} T_{7}+(\lambda-\mu) T_{6} T_{9}-T_{11} T_{16}, \\
(\mu-1) T_{3} T_{4}-\mu T_{8} T_{10}+T_{14} T_{16}, & (-\lambda+1) T_{2} T_{14}+(-\mu+1) T_{3} T_{15}+T_{10} T_{12}, \\
(\lambda \mu-\mu) T_{2} T_{8}+(-\lambda \mu+\lambda) T_{3} T_{9}-T_{12} T_{16}, & (\lambda-\mu) T_{2} T_{6}+\lambda T_{7} T_{10}-T_{13} T_{16}, \\
(\lambda-1) T_{2} T_{4}-\lambda T_{9} T_{10}-T_{15} T_{16}, & (\lambda \mu-\mu) T_{1} T_{7}+(\lambda-\mu) T_{3} T_{9}-T_{12} T_{16}, \\
(\mu-1) T_{1} T_{6}-T_{8} T_{10}+T_{14} T_{16}, & (\lambda-1) T_{1} T_{5}-T_{9} T_{10}-T_{15} T_{16}
\end{array}
$$

$\left[\begin{array}{rrrrrrrrrrrrrrrr}1 & 0 & 0 & 0 & -1 & -1 & 0 & 1 & 1 & -1 & 0 & 1 & -1 & 0 & 0 & 0 \\ 0 & 1 & 0 & 0 & 1 & 0 & 0 & -1 & 0 & 1 & 0 & 0 & 1 & 0 & 1 & 0 \\ 0 & 0 & 1 & 0 & 0 & 1 & 0 & 0 & -1 & 1 & 0 & 0 & 1 & 1 & 0 & 0 \\ 0 & 0 & 0 & 1 & 1 & 1 & 0 & 0 & 0 & 1 & 0 & -1 & 0 & 0 & 0 & 1 \\ 0 & 0 & 0 & 0 & 0 & 0 & 1 & 1 & 1 & -1 & 0 & 0 & -1 & -1 & -1 & 1 \\ 0 & 0 & 0 & 0 & 0 & 0 & 0 & 0 & 0 & 0 & 1 & 1 & 1 & 1 & 1 & -1\end{array}\right]$

In this case, $X$ is the blow up of $\mathbb{P}_{2}$ in $[1,0,0],[0,1,0],[0,0,1],[1,1,1]$ and $[1, \lambda, \mu]$ where $\lambda \neq \mu \in \mathbb{K}^{*} \backslash\{1\}$.

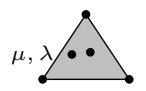

(vii) The $\mathbb{Z}^{6}$-graded ring $\mathbb{K}\left[T_{1}, \ldots, T_{11}\right] / I$, where generators for $I$ and the degree matrix are given as

$$
\begin{aligned}
& \begin{array}{l}
T_{6} T_{2} T_{4}+T_{5} T_{9}-T_{8} T_{10}, \\
T_{3} T_{4} T_{5}+T_{6} T_{7}-T_{11} T_{10},
\end{array} T_{1} T_{5}+T_{7} T_{8} T_{6}-T_{9} T_{4} T_{11}, \\
& T_{3} T_{4}^{2} T_{2}-T_{7} T_{9}-T_{1} T_{10}, \\
& {\left[\begin{array}{lllllllrrrr}
1 & 0 & 0 & 0 & 0 & 0 & 0 & 1 & 0 & -1 & 1 \\
0 & 1 & 0 & 0 & 0 & 0 & 0 & 0 & 1 & 1 & -1 \\
0 & 0 & 1 & 0 & 0 & 0 & 1 & -1 & 0 & 1 & 0 \\
0 & 0 & 0 & 1 & 0 & 0 & 1 & -1 & 1 & 2 & -1 \\
0 & 0 & 0 & 0 & 1 & 0 & 1 & 0 & -1 & 0 & 1 \\
0 & 0 & 0 & 0 & 0 & 1 & -1 & 1 & 1 & 0 & 0
\end{array}\right]}
\end{aligned}
$$

In this case, $X$ is the blow up of $\mathbb{P}_{2}$ in the points $[1,0,0],[0,1,0],[0,0,1]$, $[1,1,0]$ and $[1,0,1]$.

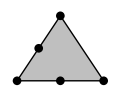

(viii) The $\mathbb{Z}^{6}$-graded ring $\mathbb{K}\left[T_{1}, \ldots, T_{10}\right] / I$, where generators for $I$ and the degree matrix are given as

$$
T_{3} T_{5} T_{8}-T_{2} T_{6}-T_{9} T_{10}, T_{1} T_{5}+T_{7} T_{8}-T_{2} T_{4} T_{10}
$$




$$
\left[\begin{array}{rlllllrrrr}
1 & 0 & 0 & 0 & 0 & 0 & 1 & 0 & -1 & 1 \\
0 & 1 & 0 & 0 & 0 & 0 & -1 & 1 & 2 & -1 \\
0 & 0 & 1 & 0 & 0 & 0 & 1 & -1 & 0 & 0 \\
0 & 0 & 0 & 1 & 0 & 0 & 0 & 0 & 1 & -1 \\
0 & 0 & 0 & 0 & 1 & 0 & 2 & -1 & -1 & 1 \\
0 & 0 & 0 & 0 & 0 & 1 & -1 & 1 & 1 & 0
\end{array}\right]
$$

In this case, $X$ is the 2 -fold blow up of $\mathbb{P}_{2}$ in $[1,0,0]$ of type $g_{1}$ and single blow ups in $[0,1,0],[0,0,1]$ and $[1,1,0]$.

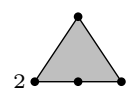

(ix) The $\mathbb{Z}^{6}$-graded ring $\mathbb{K}\left[T_{1}, \ldots, T_{10}\right] / I$, where generators for $I$ and the degree matrix are given as

$$
\begin{gathered}
T_{1} T_{5} T_{10}-T_{2} T_{6}-T_{7} T_{8}, T_{2} T_{4} T_{7}^{a-1} T_{8}^{a-2}-T_{3} T_{5}-T_{9} T_{10} \\
{\left[\begin{array}{rrrrrrrrrr}
1 & 0 & 0 & 0 & 0 & 0 & 0 & 0 & 1 & -1 \\
0 & 1 & 0 & 0 & 0 & 0 & -a+1 & a & -1 & 1 \\
0 & 0 & 1 & 0 & 0 & 0 & 1 & -1 & 1 & 0 \\
0 & 0 & 0 & 1 & 0 & 0 & -1 & 1 & 0 & 0 \\
0 & 0 & 0 & 0 & 1 & 0 & 1 & -1 & 2 & -1 \\
0 & 0 & 0 & 0 & 0 & 1 & -a+2 & a-1 & -1 & 1
\end{array}\right]}
\end{gathered}
$$

In this case, $X$ is the blow up of $\mathbb{F}_{a}$ in $[0,1,0,1],[1,0,0,1],[1,1,0,1]$ and $[0,1,1,1]$ where $a \in \mathbb{Z}_{\geq 3}$.

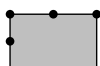

(x) The $\mathbb{Z}^{6}$-graded ring $\mathbb{K}\left[T_{1}, \ldots, T_{10}\right] / I$, where generators for $I$ and the degree matrix are given as

$$
\begin{gathered}
T_{2}^{a} T_{4}-T_{3} T_{5} T_{6}^{2} T_{10}-T_{7} T_{8}, T_{1} T_{2}^{a-1} T_{4} T_{8}-T_{3} T_{6}-T_{9} T_{10} \\
{\left[\begin{array}{rrrrrrrrrr}
1 & 0 & 0 & 0 & 0 & 0 & 1 & -1 & 0 & 0 \\
0 & 1 & 0 & 0 & 0 & 0 & 2 a-1 & -a+1 & -a & a \\
0 & 0 & 1 & 0 & 0 & 0 & -1 & 1 & 2 & -1 \\
0 & 0 & 0 & 1 & 0 & 0 & 2 & -1 & -1 & 1 \\
0 & 0 & 0 & 0 & 1 & 0 & 0 & 0 & 1 & -1 \\
0 & 0 & 0 & 0 & 0 & 1 & -1 & 1 & 3 & -2
\end{array}\right]}
\end{gathered}
$$

In this case, $X$ is the 3 -fold blow up of $\mathbb{F}_{a}, a \in \mathbb{Z}_{\geq 3}$, in $[0,1,0,1]$ of type $g_{1} s_{1}$ with the intersection point $s_{1}$ of the 1 st exceptional divisor and the transform of $V\left(T_{3}\right) \subseteq \mathbb{F}_{a}$ and a single blow up of $[0,1,1,1]$.

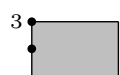

(xi) The $\mathbb{Z}^{6}$-graded ring $\mathbb{K}\left[T_{1}, \ldots, T_{10}\right] / I$, where generators for $I$ and the degree matrix are given as

$$
\begin{gathered}
T_{2}^{a} T_{4}-T_{3} T_{6} T_{10} T_{5}-T_{7} T_{8}, T_{1} T_{2}^{a-1} T_{4} T_{7} T_{8}^{2}-T_{3}^{2} T_{5}-T_{9} T_{10} \\
{\left[\begin{array}{lllllllrrrr}
1 & 0 & 0 & 0 & 0 & 0 & 1 & -1 & 0 & 0 \\
0 & 1 & 0 & 0 & 0 & 0 & 3 a-1 & -2 a+1 & -a & a \\
0 & 0 & 1 & 0 & 0 & 0 & -2 & 2 & 3 & -1 \\
0 & 0 & 0 & 1 & 0 & 0 & 3 & -2 & -1 & 1 \\
0 & 0 & 0 & 0 & 1 & 0 & -1 & 1 & 2 & -1 \\
0 & 0 & 0 & 0 & 0 & 1 & 0 & 0 & 1 & -1
\end{array}\right]}
\end{gathered}
$$

In this case, $X$ is the 3 -fold blow up of $\mathbb{F}_{a}, a \in \mathbb{Z}_{\geq 3}$, in $[0,1,0,1]$ of type $g_{2} s_{1}$ with the intersection point $s_{1}$ of the 1 st exceptional divisor and the transform of $V\left(T_{1}\right) \subseteq \mathbb{F}_{a}$ and a single blow up of $[0,1,1,1]$.

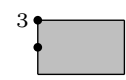

(xii) † The $\mathbb{Z}^{6}$-graded ring $\mathbb{K}\left[T_{1}, \ldots, T_{9}\right] / I$, where generators for $I$ and the degree matrix are given as

$$
\begin{gathered}
T_{1} T_{2}^{2 a-1} T_{4}^{2}-T_{3}^{2} T_{5}-T_{1} T_{2}^{a-1} T_{3} T_{4} T_{5} T_{6} T_{7} T_{9}+T_{7} T_{8} T_{9}^{2} \\
{\left[\begin{array}{llllllllrr}
1 & 0 & 0 & 0 & 1 & 0 & 0 & 3 & -1 \\
0 & 1 & 0 & 0 & 2 a-1 & 0 & 0 & 4 a-3 & -a+1 \\
0 & 0 & 1 & 0 & -2 & 0 & 0 & -2 & 1 \\
0 & 0 & 0 & 1 & 2 & 0 & 0 & 4 & -1 \\
0 & 0 & 0 & 0 & 0 & 1 & 0 & 2 & -1 \\
0 & 0 & 0 & 0 & 0 & 0 & 1 & 1 & -1
\end{array}\right]}
\end{gathered}
$$


In this case, $X$ is the 4 -fold blow up of $\mathbb{F}_{a}, a \in \mathbb{Z}_{\geq 3}$, in $[0,1,0,1]$ of type $g_{3} g_{2} s_{1}$ with the intersection point $s_{1}$ of the 1 st exceptional divisor and the transform of $V\left(T_{1}\right) \subseteq \mathbb{F}_{a}$.

(xiii) $\dagger$ The $\mathbb{Z}^{6}$-graded ring $\mathbb{K}\left[T_{1}, \ldots, T_{9}\right] / I$, where generators for $I$ and the degree matrix are given as

$$
\begin{aligned}
& T_{2}^{a} T_{4}-T_{3} T_{5} T_{6}^{2}-T_{7} T_{9} T_{1} T_{2}^{a-1} T_{4} T_{5} T_{6}+T_{7} T_{9}^{2} T_{8} \\
& {\left[\begin{array}{rrrrrrrrr}
1 & 1 & 0 & -a & 0 & 0 & 0 & 0 & 0 \\
-1 & 0 & -1 & 0 & 1 & 0 & 0 & 0 & 0 \\
-1 & 0 & -2 & 0 & 0 & 1 & 0 & 0 & 0 \\
-1 & 0 & 1 & 1 & 0 & 0 & 1 & 0 & 0 \\
0 & 0 & 1 & 1 & 0 & 0 & 0 & 1 & 0 \\
-1 & 0 & 2 & 2 & 0 & 0 & 0 & 0 & 1
\end{array}\right]}
\end{aligned}
$$

In this case, $X$ is the 2-fold blow up of $\mathbb{F}_{a}$, a $\in \mathbb{Z}_{\geq 3}$, in $[0,1,0,1]$ of type $s_{1}$ with the intersection point $s_{1}$ of the 1 st exceptional divisor and the transform of $V\left(T_{3}\right) \subseteq \mathbb{F}_{a}$ and the 2 -fold blow up of $[0,1,1,1]$ of type $g_{1}$.

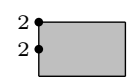

(xiv) t† The $\mathbb{Z}^{6}$-graded ring $\mathbb{K}\left[T_{1}, \ldots, T_{11}\right] / I$, where generators for $I$ and the degree matrix are given as

$$
\begin{aligned}
& \begin{array}{lc}
T_{7} T_{8}-T_{2}^{a} T_{4} T_{6}^{a-1} T_{11}^{a}+T_{3} T_{5}, & T_{9} T_{11}-T_{1}^{a} T_{4} T_{5}^{a} T_{8}^{a-1}-\kappa T_{6} T_{7}, \\
T_{10} T_{11}-T_{1}^{a} T_{4} T_{5}^{a-1} T_{8}^{a}+\kappa T_{3} T_{6}, & -\kappa T_{2}^{a} T_{4} T_{6}^{a} T_{11}^{a-1}+T_{8} T_{9}-T_{5} T_{10},
\end{array} \\
& T_{1}^{a} T_{2}^{a} T_{4}^{2} T_{5}^{a-1} T_{6}^{a-1} T_{8}^{a-1} T_{11}^{a-1}-T_{3} T_{9}-T_{7} T_{10} \\
& {\left[\begin{array}{rrrrrrrrrrr}
1 & 0 & 0 & 0 & 0 & 0 & 1 & -1 & 1 & 0 & 0 \\
0 & 1 & 0 & 0 & 0 & 0 & 0 & 0 & 1 & 1 & -1 \\
0 & 0 & 1 & 0 & 0 & a-1 & 0 & 1 & 2 a-3 & 2 a-2 & -a+2 \\
0 & 0 & 0 & 1 & 0 & 1 & 0 & 0 & 2 & 2 & -1 \\
0 & 0 & 0 & 0 & 1 & a-1 & 1 & 0 & 2 a-2 & 2 a-3 & -a+2 \\
0 & 0 & 0 & 0 & 0 & a & -1 & 1 & 2 a-2 & 2 a-1 & -a+1
\end{array}\right]}
\end{aligned}
$$

In this case, $X$ is the blow up of $\mathbb{F}_{a}, a \in \mathbb{Z}_{\geq 3}$, in $[0,1,0,1],[1,0,0,1]$, $[0,1,1,1],[1,0,1, \kappa]$ where $\kappa \in \mathbb{K}^{*}$.

Of the surfaces marked with a single $\dagger$ we do not know whether they admit a $\mathbb{K}^{*}$ action. The surface marked with $\dagger \dagger$ does not admit a non-trivial $\mathbb{K}^{*}$-action and the listed ring is the Cox ring for $a \leq 15$, whereas for a $>15$, the surface is a Mori dream surface having the $H_{2}$-equivariant normalization of $\mathbb{K}\left[T_{1}, \ldots, T_{11}\right] / I:\left(T_{1} \cdots T_{11}\right)^{\infty}$ as its Cox ring.

Remark 6.2. In Theorem 6.1 surface (vi) is a smooth del Pezzo surface of degree 4. The surfaces (iii), $(v)$, (vii), (viii) are weak del Pezzo surfaces of degree 4 of singularity type $A_{3}, A_{1}, 2 A_{1}, A_{2}$ respectively. The number of generators and relations of their Cox rings was given in [20, Table 6.2]. All other surfaces listed in the table of Theorem 6.1 contain $(-k)$-curves with $k \geq 3$ and therefore are not weak del Pezzo surfaces.

Remark 6.3. The Cox ring generators occurring in Theorem 6.1, are either contractible curves on $X$ or they define curves in $\mathbb{P}_{2}$ or, in the case of a blown up $\mathbb{F}_{a}$, in $\mathbb{P}(1,1, a)$, where their degrees are given as follows (the contractible ones are indicated by "-"):

\begin{tabular}{ll}
\hline surface & generator degrees in $\mathbb{P}_{2}$ or $\mathbb{P}(1,1, a)$ \\
\hline (i) & $1,1,1,-,-, 2,-,-, 4,-$ \\
(ii) & $1,1,1,-,-,-, 1,-, 1,-$ \\
(iii) & $1,1,1,-,-,-, 1,-, 2,2,-$ \\
(iv) & $1,1,1,-,-,-, 1,-, 2,-$ \\
(v) & $1,1,1,-,-,-, 1,1,1,-, 1,1,-$ \\
(vi) & $1,1,1,-,-,-, 1,1,1,-, 1,2,1,1,1,-$ \\
(vii) & $1,1,1,-,-,-, 1,-, 1,1,-$ \\
(viii) & $1,1,1,-,-,-, 1,-, 1,-$
\end{tabular}




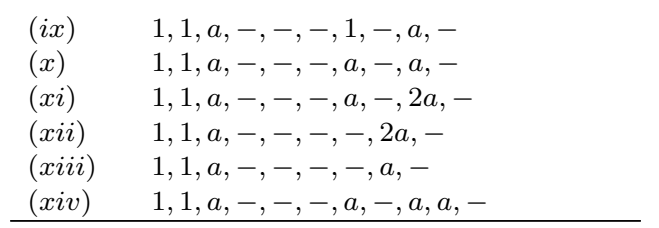

Lemma 6.4. Consider Setting 2.5. Assume that $X_{1} \subseteq Z_{1}$ is a CEMDS, $Z_{2} \rightarrow Z_{1}$ arises from a barycentric subdivision of a regular cone $\sigma \in \Sigma_{1}$ and $X_{2} \rightarrow X_{1}$ has as center a point $x \in X_{1} \cap \mathbb{T}^{n} \cdot z_{\sigma}$. Let $f$ be the product over all $T_{i}$, where $P_{1}\left(e_{i}\right) \notin \sigma$, and choose $z \in \mathbb{K}^{r_{1}}$ with $p_{1}(z)=x$. Then $X_{2} \rightarrow X_{1}$ is the blow up at $x$ provided we have

$$
\left\langle T_{i} ; z_{i}=0\right\rangle_{f}+I\left(P_{1}, z\right)_{f}=\left\langle T_{i} ; e_{i} \in \widehat{\sigma}\right\rangle_{f}+I\left(\overline{X_{1}}\right)_{f} \subseteq \mathbb{K}\left[T_{1}, \ldots, T_{r_{1}}\right]_{f} .
$$

Proof. Let $Z_{1, \sigma} \subseteq Z_{1}$ be the affine chart given by $\sigma$ and set $X_{1, \sigma}:=X_{1} \cap Z_{1, \sigma}$. In order to see that the toric blow up $Z_{2} \rightarrow Z_{1}$ induces a blow up $X_{2} \rightarrow X_{1}$, we have to show

$$
\mathfrak{m}_{x}=I\left(\mathbb{T}^{n} \cdot z_{\sigma}\right)+I\left(X_{1, \sigma}\right) \subseteq \Gamma\left(Z_{1, \sigma}, \mathcal{O}\right) .
$$

Consider the quotient map $p_{1}: \widehat{Z}_{1} \rightarrow Z_{1}$. Then we have $p_{1}^{-1}\left(Z_{1, \sigma}\right)=\mathbb{K}_{f}^{r_{1}}$ and, since $\sigma$ is regular, $\Gamma\left(p_{1}^{-1}\left(Z_{1, \sigma}\right), \mathcal{O}\right)$ admits units in every $K_{1}$-degree. This implies

$$
\begin{gathered}
p_{1}^{*}\left(\mathfrak{m}_{x}\right)=\left\langle T_{i} ; z_{i}=0\right\rangle_{f}+I\left(P_{1}, z\right)_{f}, \\
p_{1}^{*}\left(I\left(\mathbb{T}^{n} \cdot z_{\sigma}\right)\right)=\left\langle T_{i} ; e_{i} \in \widehat{\sigma}\right\rangle_{f}, \quad p_{1}^{*}\left(I\left(X_{1, \sigma}\right)\right)=I\left(\overline{X_{1}}\right)_{f} .
\end{gathered}
$$

Consequently, the assumption together with injectivity of the pullback map $p_{1}^{*}$ give the assertion.

Proof of Theorem 6.1. The idea is a stepwise classification of the Cox rings for all smooth rational surfaces of Picard number $\varrho(X)=1, \ldots, 6$. For $\varrho(X) \leq 5$, it is possible to list all occurring Cox rings up to isomorphism. See 33 for the full list. We treat exemplarily the surface $(x)$ in the table of the theorem, which is obtained as blow up of a $\mathbb{K}^{*}$-surface $X_{1}$ with $\varrho\left(X_{1}\right)=5$. The Cox ring and degree matrix $Q_{1}$ of $X_{1}$ are

$$
\mathbb{K}\left[T_{1}, \ldots, T_{8}\right] /\left\langle T_{2}^{a} T_{4}-T_{3} T_{5} T_{6}^{2}-T_{7} T_{8}\right\rangle, \quad\left[\begin{array}{rrrrrrrr}
1 & 1 & 0 & -a & 0 & 0 & 0 & 0 \\
-1 & 0 & -1 & 0 & 1 & 0 & 0 & 0 \\
-1 & 0 & -2 & 0 & 0 & 1 & 0 & 0 \\
0 & 0 & 1 & 1 & 0 & 0 & 1 & 0 \\
-1 & 0 & 1 & 1 & 0 & 0 & 0 & 1
\end{array}\right] .
$$

The surface $X_{2}$ is the blow up of $X_{1}$ in the point with Cox coordinates $q:=$ $(1,1,1,1,0,1,1,1) \in \bar{X}_{1}$. To compute the Cox ring of $X_{2}$, we formally apply the steps of Algorithm 5.4. In Setting 2.9, we choose the embedding

$$
\bar{\iota}: \mathbb{K}^{8} \rightarrow \mathbb{K}^{9}, \quad z \mapsto\left(z, f_{1}(z)\right), \quad f_{1}:=T_{1} T_{2}^{a-1} T_{4} T_{8}-T_{3} T_{6} .
$$

The new degree matrix $Q_{1}^{\prime}$ and the matrix $P_{1}^{\prime}$, whose columns are generators for the rays of the fan $\Sigma_{1}^{\prime}$ of the ambient toric variety $Z_{1}^{\prime}$, are

$$
Q_{1}^{\prime}=\left[\begin{array}{r|r}
0 \\
Q_{1} \\
-1 \\
-1 \\
1 \\
1
\end{array}\right], \quad P_{1}^{\prime}=\left[\begin{array}{rrrrrrrrr}
1 & a-1 & 0 & 1 & 0 & 0 & 0 & 1 & -1 \\
0 & a & 0 & 1 & 0 & 0 & -1 & -1 & 0 \\
0 & 0 & 1 & 0 & 0 & 1 & 0 & 0 & -1 \\
0 & 0 & 0 & 0 & 1 & 1 & -1 & -1 & 1
\end{array}\right]
$$

To blow up the point on $X_{1}^{\prime}$ with Cox coordinates $\bar{\iota}(q)=(1,1,1,1,0,1,1,1,0) \in \bar{X}_{1}^{\prime}$, we perform the toric modification $\pi: Z_{2} \rightarrow Z_{1}^{\prime}$ given by the stellar subdivision $\Sigma_{2} \rightarrow \Sigma_{1}^{\prime}$ at $v:=(-1,0,-1,2) \in \mathbb{Z}^{4}$. Note that this completes the first threes steps of Algorithm 5.4. For the fourth one, we now formally apply Algorithm 3.6. Let $P_{2}:=\left[P_{1}^{\prime}, v\right]$. The ideal $I_{2} \subseteq \mathbb{K}\left[T_{1}, \ldots, T_{10}\right]$ of $\bar{X}_{2}$ is generated by

$$
\begin{aligned}
g_{1}:= & p_{2}^{\sharp}\left(p_{1}\right)_{\sharp}\left(T_{2}^{a} T_{4}-T_{3} T_{5} T_{6}^{2}-T_{7} T_{8}\right)=T_{2}^{a} T_{4}-T_{3} T_{5} T_{6}^{2} T_{10}-T_{7} T_{8}, \\
& g_{2}:=p_{2}^{\sharp}\left(p_{1}\right)_{\sharp}\left(T_{9}-f_{1}\right)=T_{1} T_{2}^{a-1} T_{4} T_{8}-T_{3} T_{6}-T_{9} T_{10} .
\end{aligned}
$$


We show that $I_{2}$ is prime; this implies in particular that $I_{2}$ is saturated with respect to $T_{10}$. On the open subset

$$
U:=\left\{x \in \bar{X}_{2} ; x_{8} x_{9} \neq 0 \text { or } x_{7} x_{10} \neq 0\right\} \subseteq \bar{X}_{2}
$$

the Jacobian $\left(\partial g_{i} / \partial T_{j}\right)_{i, j}$ is of rank two and $U$ is a subset of the union of the 8-dimensional subspaces

$$
V\left(\mathbb{K}^{10} ; T_{8}, T_{7}\right), \quad V\left(\mathbb{K}^{10} ; T_{8}, T_{10}\right), \quad V\left(\mathbb{K}^{10} ; T_{9}, T_{7}\right), \quad V\left(\mathbb{K}^{10} ; T_{9}, T_{10}\right),
$$

all of which have six-dimensional intersection with $\bar{X}_{2}$. Hence, $\bar{X}_{2} \backslash U$ is of codimension at least two in $\bar{X}_{2}$. Furthermore, since the effective cone of $X_{2}$ is pointed, $\bar{X}_{2}$ is connected. By Serre's criterion the ideal $I_{2}$ is prime, see e.g. 32.

We claim that in $R_{2}=\mathbb{K}\left[T_{1}, \ldots, T_{10}\right] / I_{2}$, the variable $T_{10}$ defines a prime element. Instead of showing that $I_{2}+\left\langle T_{10}\right\rangle \subseteq \mathbb{K}\left[T_{1}, \ldots, T_{10}\right]$ is prime, removing non-used variables, we may show this for

$$
I_{0}:=\left\langle T_{2}^{a} T_{4}-T_{6} T_{7}, T_{1} T_{2}^{a-1} T_{4} T_{7}-T_{3} T_{5}\right\rangle \subseteq \mathbb{K}\left[T_{1}, \ldots, T_{7}\right] .
$$

Considered as an ideal in $\mathbb{K}\left[T_{1}^{ \pm 1}, \ldots, T_{7}^{ \pm 1}\right]$, it is prime since the matrix with the exponents of the binomial generators as its rows

$$
\left[\begin{array}{rrrrrrr}
0 & a & 0 & 1 & 0 & -1 & -1 \\
1 & a-1 & -1 & 1 & -1 & 0 & 1
\end{array}\right]
$$

has Smith normal form $\left[E_{2}, 0\right]$, where $E_{2}$ is the $2 \times 2$ unit matrix. Now, by Lemma 3.3, $I_{0}$ is prime if $I_{0}=I_{0}:\left(T_{1} \cdots T_{7}\right)^{\infty}$. To this end, the set

$$
\mathcal{G}:=\left\{T_{1} T_{6} T_{7}^{2}-T_{2} T_{3} T_{5}, T_{2}^{a} T_{4}-T_{6} T_{7}, T_{1} T_{2}^{a-1} T_{4} T_{7}-T_{3} T_{5}\right\} \subseteq I_{0}
$$

turns out to be a Gröbner basis for $I_{0}$ with respect to the degree reverse lexicographical ordering for any ordering of the variables. By [44, Lem. 12.1], we know that

$$
\left\{\frac{f}{T_{i}^{k_{i}(f)}} ; f \in \mathcal{G}\right\}=\mathcal{G}, \quad k_{i}(f):=\max \left(n \in \mathbb{Z}_{\geq 0} ; T_{i}^{n} \mid f\right)
$$

is a Gröbner basis for $I_{0}: T_{i}^{\infty}$ for any $i$. As in [44, p. 114], the claim follows from

$$
I_{0}:\left(T_{1} \cdots T_{7}\right)^{\infty}=\left(\left(\cdots\left(I_{0}: T_{1}^{\infty}\right) \cdots\right): T_{7}^{\infty}\right)=I_{0} .
$$

Moreover, no two variables $T_{i}, T_{j}$ are associated since $\operatorname{deg} T_{i} \neq \operatorname{deg} T_{j}$ for all $i, j$ and $T_{10} \nmid T_{i}$ for all $i<10$ because $\operatorname{dim} \bar{X}_{2} \cap V\left(T_{i}, T_{10}\right)$ is at most six for each $i$. For instance,

$$
\bar{X}_{2} \cap V\left(T_{5}, T_{10}\right)=V\left(T_{10}, T_{5}, T_{2}^{a} T_{4}-T_{7} T_{8}, T_{1} T_{2}^{a-1} T_{4} T_{8}-T_{3} T_{6}\right) \subseteq \mathbb{K}^{10}
$$

is of dimension six on $\mathbb{T}^{10} \cdot(1,1,1,1,0,1,1,1,1,0)$ since its dimension equals 8 rank $B$ with

$$
B:=\left[\begin{array}{rrrrrrrrrr}
0 & a & 0 & 1 & 0 & 0 & -1 & -1 & 0 & 0 \\
1 & a-1 & -1 & 1 & 0 & -1 & 0 & 1 & 0 & 0
\end{array}\right]
$$

having the exponents of the binomial equations as its rows. Similarly, on the smaller tori, the dimension is at most six. By Theorem 2.6 and Algorithm 3.6, $R_{2}$ is the Cox ring of the performed modification. We claim that we performed the desired blow up. The ideal

$$
I^{\prime}:=\left\langle T_{5}, T_{9}, f_{1}, T_{2}^{a} T_{4}-T_{7} T_{8}\right\rangle \subseteq \mathbb{K}\left[T_{1}, \ldots, T_{9}\right]
$$

is prime since $I_{0}$ is prime, $\bar{\iota}(q) \in V\left(I^{\prime}\right) \subseteq \mathbb{K}^{9}$ and, as seen above,

$$
\operatorname{dim} V\left(\mathbb{K}^{9} ; I^{\prime}\right)=-1+\operatorname{dim} \bar{X}_{2} \cap V\left(\mathbb{K}^{10} ; T_{5}, T_{10}\right)=5 .
$$

By [39, Thm. 7.4], as $K_{1}^{\prime}$ is free, $I\left(P_{1}^{\prime}, \bar{\iota}(q)\right)$ is prime. This implies $I^{\prime}=I\left(P_{1}^{\prime}, \bar{\iota}(q)\right)$. Lemma 6.4 applies. The Cox ring and degree matrix of $X_{2}$ are listed in the table under $(x)$. Note that $X_{2}$ is not a $\mathbb{K}^{*}$-surface: by the blow up sequence, its graph of negative curves contains the subgraph 


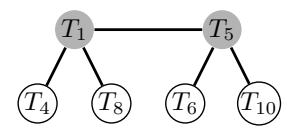

where, by the theory of $\mathbb{K}^{*}$-surfaces [41], the curves corresponding to $T_{1}$ and $T_{5}$ must correspond to the sink and source of the $\mathbb{K}^{*}$-action. On a $\mathbb{K}^{*}$-surface, sink and source must not meet.

\section{LiNEAR GENERATION}

We consider the blow up $X$ of a projective space $\mathbb{P}_{n}$ at $k$ distinct points $x_{1}, \ldots, x_{k}$, where $k>n+1$. Our focus is on special configurations in the sense that the Cox ring of $X$ is generated by the exceptional divisors and the proper transforms of hyperplanes. We assume that $x_{1}, \ldots, x_{n+1}$ are the standard toric fixed points, i.e. we have

$$
x_{1}=[1,0, \ldots, 0], \quad \ldots, \quad x_{n+1}=[0, \ldots, 0,1] .
$$

Now, write $\mathcal{P}:=\left\{x_{1}, \ldots, x_{k}\right\}$ and let $\mathcal{L}$ denote the set of all hyperplanes $\ell \subseteq \mathbb{P}_{n}$ containing $n$ (or more) points of $\mathcal{P}$. For every $\ell \in \mathcal{L}$, we fix a linear form $f_{\ell} \in$ $\mathbb{K}\left[T_{1}, \ldots, T_{n+1}\right]$ with $\ell=V\left(f_{\ell}\right)$. Note that the $f_{\ell}$ are homogeneous elements of degree one in the Cox ring of $\mathbb{P}_{n}$.

The idea is now to take all $T_{\ell}$, where $\ell \in \mathcal{L}$, as prospective generators of the Cox ring of the blow up $X$ and then to compute the Cox ring using Algorithms 3.1, 3.6 and 3.2. Here comes the algorithmic formulation.

Algorithm 7.1 (LinearBlowUp). Input: a collection $x_{1}, \ldots, x_{k} \in \mathbb{P}_{n}$ of pairwise distinct points.

- Set $X_{1}:=\mathbb{P}_{n}$, let $\Sigma_{1}$ be the fan of $\mathbb{P}_{n}$ and $P_{1}$ the matrix with columns $e_{0}, \ldots, e_{n}$, where $e_{0}=-\left(e_{1}+\ldots+e_{n}\right)$.

- Compute the set $\mathcal{L}$ of all hyperplanes through any $n$ of the points $x_{1}, \ldots, x_{k}$, let $\left(f_{\ell} ; \ell \in \mathcal{L}^{\prime}\right)$ be the collection of the $f_{\ell}$ different from all $T_{i}$.

- Compute the stretched CEMDS $\left(P_{1}^{\prime}, \Sigma_{1}^{\prime}, G_{1}^{\prime}\right)$ by applying Algorithm 3.1 to $\left(P_{1}, \Sigma_{1}, G_{1}\right)$ and $\left(f_{\ell} ; \ell \in \mathcal{L}^{\prime}\right)$.

- Determine the Cox coordinates $z_{i}^{\prime} \in \mathbb{K}^{r_{1}^{\prime}}$ of the points $x_{i}^{\prime} \in X_{1}^{\prime}$ corresponding to $x_{i} \in X_{1}$.

- Let $\Sigma_{2}$ be the barycentric subdivision of $\Sigma_{1}^{\prime}$ at the cones $\sigma_{i}^{\prime}$, corresponding to the toric orbits containing $x_{i}^{\prime}=p_{1}^{\prime}\left(z_{i}^{\prime}\right)$. Write primitive generators for the rays of $\Sigma_{2}$ into a matrix $P_{2}=\left[P_{1}, B\right]$.

- Compute $\left(P_{2}, \Sigma_{2}, G_{2}\right)$ by applying Algorithm 3.6 to $\left(P_{1}^{\prime}, \Sigma_{1}^{\prime}, G_{1}^{\prime}\right)$ and the pair $\left(P_{2}, \Sigma_{2}\right)$

- Set $\left(P_{2}^{\prime}, \Sigma_{2}^{\prime}, G_{2}^{\prime}\right):=\left(P_{2}, \Sigma_{2}, G_{2}\right)$. Eliminate all fake relations by applying Algorithm 3.2 with option verify. Call the output $\left(P_{2}, \Sigma_{2}, G_{2}\right)$.

Output: $\left(P_{2}, \Sigma_{2}, G_{2}\right)$. If the verifications in the last step were positive, this is a CEMDS describing the blow up of $\mathbb{P}_{n}$ at the points $x_{1}, \ldots, x_{k}$; in particular the $K_{2}$-graded algebra $R_{2}$ is the Cox ring of $X_{2}$.

Lemma 7.2. In Algorithm 7.1 for each $x_{i}$ the barycentric subdivision of $\sigma_{i}^{\prime}$ induces a blow up of $X_{1}^{\prime}$ in $x_{i}^{\prime}$.

Proof. In Algorithm 7.1. let $G_{1}^{\prime}=\left\{T_{n+1+j}-f_{j} ; 1 \leq j \leq s\right\}$. We have

$$
\overline{X_{1}^{\prime}} \cap V\left(T_{j} ; e_{j} \in \widehat{\sigma}_{i}^{\prime}\right) \supseteq V\left(I\left(P_{1}^{\prime}, z_{i}^{\prime}\right)\right)=\overline{H_{1}^{\prime} \cdot z_{i}^{\prime}}
$$

since the left hand side is $H_{1}^{\prime}$-invariant. Equality is achieved by comparing dimensions and the fact that $x_{i}$ is cut out by $n$ hyperplanes. Taking ideals, this implies that $I\left(\overline{X_{1}^{\prime}}\right)+\left\langle T_{j} ; e_{j} \in \widehat{\sigma}_{i}^{\prime}\right\rangle$ equals $I\left(P_{1}^{\prime}, z_{i}^{\prime}\right)$ because the ideals are linear and thus radical. Since $\sigma_{i}^{\prime}$ is smooth, we may use Lemma 6.4 . 
Proof of Algorithm 7.1. By Lemma 7.2, $X_{2} \rightarrow X_{1}^{\prime}$ is the blow up at $x_{1}^{\prime}, \ldots, x_{k}^{\prime}$. It remains to show that the input ring $R_{2}^{\prime}$ of the last step is normal; this is necessary for Algorithm 3.2 We only treat the case $k=1$. Consider the stretched ring $R_{1}^{\prime}$ obtained from the third step and the ring $R_{2}$ obtained after the sixth step

$$
R_{1}^{\prime}=\mathbb{K}\left[T_{1}, \ldots, T_{r_{1}^{\prime}}\right] /\left\langle G_{1}^{\prime}\right\rangle, \quad R_{2}=\mathbb{K}\left[T_{1}, \ldots, T_{r_{1}^{\prime}}, T_{r_{2}}\right] /\left\langle G_{2}\right\rangle,
$$

where $T_{r_{2}}$ corresponds to the exceptional divisor. We assume that of the $r_{1}^{\prime}-r_{1}$ new equations $T_{i}-f_{i}$ in $\left\langle G_{1}^{\prime}\right\rangle$ the last $l$ will result in fake relations in $\left\langle G_{2}\right\rangle$. Localizing and passing to degree zero, we are in the situation

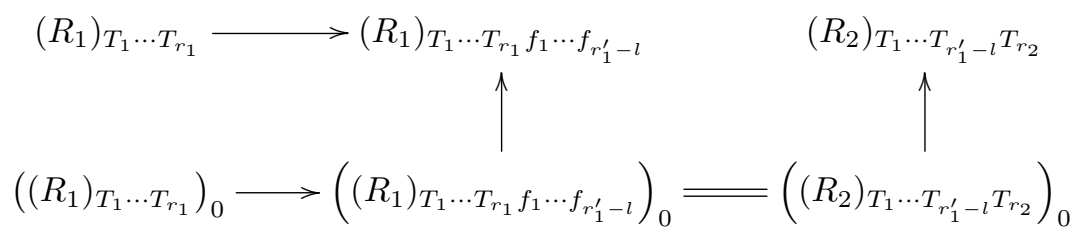

The upper left ring is $K_{1}$-factorial by assumption. By [8, Thm. 1.1] the middle ring in the lower row is a UFD and the ring on the upper right is $K_{2}$-factorial. Thus, $R_{2}$ is $K_{2}$-factorial. Since $K_{1}$ is free, also $K_{2}$ is, so $R_{2}$ is a UFD. In particular, $R_{2}$ is normal and we may apply Algorithm 3.2 .

Before eliminating fake relations, the ideal of the intersection of $\bar{X}_{2}$ with the ambient big torus $\mathbb{T}^{r_{2}}$ admits the following description in terms of incidences.

Remark 7.3. At the end of the fifth step in Algorithm 7.1, the Cox ring of $Z_{2}$ is the polynomial ring $\mathbb{K}\left[T_{\ell}, S_{p}\right]$ with indices $\ell \in \mathcal{L}$ and $p \in \mathcal{P}$. Consider the homomorphism

$$
\beta: \mathbb{K}\left[T_{\ell} ; \ell \in \mathcal{L}\right] \rightarrow \mathbb{K}\left[T_{\ell}, S_{p} ; \ell \in \mathcal{L}, p \in \mathcal{P}\right], \quad T_{\ell} \mapsto T_{\ell} \cdot \prod_{p \in \ell} S_{p}
$$

Then the extension of the ideal $I_{2} \subseteq \mathbb{K}\left[T_{\ell}, S_{p}\right]$ to the Laurent polynomial ring $\mathbb{K}\left[T_{\ell}^{ \pm}, S_{p}^{ \pm}\right]$is generated by $\beta\left(T_{\ell}-f_{\ell}\right)$, where $\ell \in \mathcal{L}^{\prime}$.

Remark 7.4. Some geometric properties of the blow up $X$ may be seen directly from the combinatorics of the underlying finite linear space $\mathfrak{L}=(\mathcal{P}, \mathcal{L}, \in)$, compare e.g. [6]. For instance, if $\mathfrak{L}$ is an $n$-design, i.e. each line $\ell \in \mathcal{L}$ contains exactly $n$ points, the ideal of the Cox ring of $X$ is classically homogeneous. Furthermore, for a surface $X$, if all points but one lie on a common line, i.e. $\mathfrak{L}$ is a near-pencil, then $X$ comes with a $\mathbb{K}^{*}$-action. It would be interesting to see further relations between $X$ and $\mathfrak{L}$.

Example 7.5. Let $X$ be the blow up of $\mathbb{P}_{2}$ in the seven points

$$
\begin{array}{ll}
x_{1}:=[1,0,0], & x_{2}:=[0,1,0], \quad x_{3}:=[0,0,1], \\
x_{4}:=[1,1,0], & x_{5}:=[1,0,-1], \quad x_{6}:=[0,1,1], \\
x_{7}:=[1,1,1] . &
\end{array}
$$

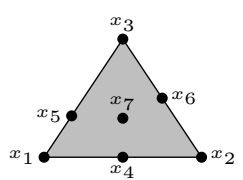

Write $S_{i}$ for the variables corresponding to $x_{i}$ and let $T_{1}, \ldots, T_{9}$ correspond to the nine lines in $\mathcal{L}$. Algorithm 7.1 provides us with the Cox ring of $X$. It is given as 
the factor ring $\mathbb{K}\left[T_{1}, \ldots, T_{9}, S_{1}, \ldots, S_{7}\right] / I$ where $I$ is generated by

$$
\begin{array}{lr}
2 T_{8} S_{4} S_{6}-T_{5} S_{2}+T_{9} S_{7}, & 2 T_{1} S_{3} S_{6}+T_{5} S_{5}-T_{6} S_{7}, \\
2 T_{4} S_{1} S_{6}+T_{6} S_{2}-T_{9} S_{5}, & -T_{1} S_{2} S_{6}+T_{2} S_{1} S_{5}-T_{7} S_{4} S_{7}, \\
2 T_{7} S_{3} S_{4}+T_{6} S_{2}+T_{9} S_{5}, & -T_{2} S_{5} S_{3}+T_{3} S_{4} S_{2}-T_{4} S_{7} S_{6}, \\
2 T_{3} S_{1} S_{4}+T_{5} S_{5}+T_{6} S_{7}, & T_{1} S_{2} S_{3}+T_{8} S_{4} S_{5}+T_{4} S_{1} S_{7}, \\
2 T_{2} S_{1} S_{3}+T_{5} S_{2}+T_{9} S_{7}, & T_{2} T_{6} S_{3}-T_{3} T_{9} S_{4}-T_{4} T_{5} S_{6}, \\
T_{3} S_{1} S_{2}+T_{8} S_{5} S_{6}-T_{7} S_{3} S_{7}, & T_{3} T_{9} S_{1}-T_{5} T_{7} S_{3}-T_{6} T_{8} S_{6}, \\
T_{2} T_{6} S_{1}-T_{5} T_{7} S_{4}+T_{1} T_{9} S_{6}, & T_{4} T_{5} S_{1}+T_{1} T_{9} S_{3}+T_{6} T_{8} S_{4}, \\
T_{3} T_{7} S_{4}^{2}+T_{1} T_{4} S_{6}^{2}+T_{2} T_{6} S_{5}, & T_{2} T_{7} S_{3}^{2}+T_{4} T_{8} S_{6}^{2}+T_{3} T_{9} S_{2}, \\
T_{1} T_{2} S_{3}^{2}+T_{3} T_{8} S_{4}^{2}-T_{4} T_{5} S_{7}, & T_{1} T_{3} S_{2}^{2}+T_{2} T_{8} S_{5}^{2}+T_{4} T_{7} S_{7}^{2}, \\
T_{3} T_{4} S_{1}^{2}+T_{1} T_{7} S_{3}^{2}-T_{6} T_{8} S_{5}, & T_{2} T_{4} S_{1}^{2}+T_{7} T_{8} S_{4}^{2}-T_{1} T_{9} S_{2}, \\
T_{2} T_{3} S_{1}^{2}+T_{1} T_{8} S_{6}^{2}+T_{5} T_{7} S_{7}, & T_{4} T_{5}^{2} T_{7}+T_{2} T_{6}^{2} T_{8}+T_{1} T_{3} T_{9}^{2}
\end{array}
$$

and the $\mathbb{Z}^{8}$-grading is given by the degree matrix

$$
\left[\begin{array}{rrrrrrrrrrrrrrrr}
0 & -1 & -1 & -1 & 0 & 0 & 0 & 0 & 0 & 1 & 0 & 0 & 0 & 0 & 0 & 0 \\
-1 & 0 & -1 & 0 & -1 & -1 & 0 & 0 & 0 & 0 & 1 & 0 & 0 & 0 & 0 & 0 \\
0 & 0 & -1 & 0 & 0 & 0 & 1 & 1 & 1 & 1 & 1 & 0 & 0 & 0 & 0 & 0 \\
-1 & -1 & 0 & 0 & 0 & 0 & -1 & 0 & 0 & 0 & 0 & 1 & 0 & 0 & 0 & 0 \\
0 & 0 & -1 & 0 & 0 & 0 & -1 & -1 & 0 & 0 & 0 & 0 & 1 & 0 & 0 & 0 \\
0 & 0 & 0 & 1 & -1 & 0 & 1 & 0 & 0 & 0 & 1 & 0 & 0 & 1 & 0 & 0 \\
-1 & 0 & 0 & -1 & 0 & 0 & 0 & -1 & 0 & 0 & 0 & 0 & 0 & 0 & 1 & 0 \\
0 & 1 & 0 & 0 & 0 & -1 & 0 & 1 & 0 & 0 & 1 & 0 & 0 & 0 & 0 & 1
\end{array}\right]
$$

The following theorem concerns blow ups of $\mathbb{P}_{3}$ in six points $x_{1}, \ldots, x_{6}$. As before, we assume that $x_{1}, \ldots, x_{4}$ are the standard toric fixed points. We call the point configuration edge-special if at least one point of $\left\{x_{5}, x_{6}\right\}$ is contained in two different hyperplanes spanned by the other points.

Theorem 7.6. Let $X$ be the blow up of $\mathbb{P}_{3}$ at distinct points $x_{1}, \ldots, x_{6}$ not contained in a hyperplane. Then $X$ is a Mori dream space. Moreover, for the following typical edge-special configurations, we obtain:

(i) For $x_{5}:=[1,1,0,0], x_{6}:=[0,1,1,1]$, the Cox ring of $X$ is $\mathbb{K}\left[T_{1}, \ldots, T_{16}\right] / I$, where $I$ is generated by

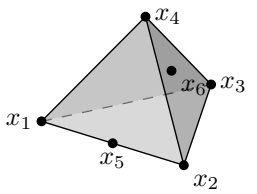

$$
\begin{array}{lr}
2 T_{4} T_{13}-2 T_{5} T_{16}-2 T_{3} T_{14}, & T_{4} T_{12} T_{15}-T_{2} T_{14}-T_{6} T_{16}, \\
T_{5} T_{12} T_{15}-T_{6} T_{13}+T_{7} T_{14}, & T_{3} T_{12} T_{15}-T_{2} T_{13}-T_{7} T_{16}, \\
T_{5} T_{11} T_{12}-T_{9} T_{13}+T_{10} T_{14}, & T_{4} T_{11} T_{12}-T_{8} T_{14}-T_{9} T_{16}, \\
T_{3} T_{11} T_{12}-T_{8} T_{13}-T_{10} T_{16}, T_{1} T_{12} T_{13}+T_{7} T_{11}-T_{10} T_{15}, \\
T_{1} T_{12} T_{14}+T_{6} T_{11}-T_{9} T_{15}, & T_{1} T_{12} T_{16}-T_{2} T_{11}+T_{8} T_{15}, \\
T_{5} T_{8}-T_{3} T_{9}+T_{4} T_{10}, & T_{2} T_{5}-T_{3} T_{6}+T_{4} T_{7}, \\
T_{1} T_{5} T_{12}^{2}+T_{7} T_{9}-T_{6} T_{10}, & T_{1} T_{3} T_{12}^{2}+T_{7} T_{8}-T_{2} T_{10}, \\
T_{1} T_{4} T_{12}^{2}+T_{6} T_{8}-T_{2} T_{9} &
\end{array}
$$

with the $\mathbb{Z}^{7}$-grading given by the degree matrix

$\left[\begin{array}{rrrrrrrrrrrrrrrr}1 & 1 & 1 & 1 & 1 & 1 & 1 & 1 & 1 & 1 & 0 & 0 & 0 & 0 & 0 & 0 \\ 0 & -1 & -1 & -1 & -1 & -1 & -1 & 0 & 0 & 0 & 1 & 0 & 0 & 0 & 0 & 0 \\ -1 & 0 & -1 & -1 & -1 & 0 & 0 & 0 & 0 & 0 & 0 & 1 & 0 & 0 & 0 & 0 \\ -1 & -1 & 0 & -1 & 0 & -1 & 0 & -1 & -1 & 0 & 0 & 0 & 1 & 0 & 0 & 0 \\ 0 & 0 & 0 & 1 & 1 & 1 & 0 & 0 & 1 & 0 & 0 & 0 & 0 & 1 & 0 & 0 \\ 1 & 1 & 0 & 0 & 0 & 1 & 1 & 0 & 0 & 0 & 0 & 0 & 0 & 0 & 1 & 0 \\ 0 & 1 & 1 & 1 & 0 & 0 & 0 & 1 & 0 & 0 & 0 & 0 & 0 & 0 & 0 & 1\end{array}\right]$.

(ii) For $x_{5}:=[2,1,0,0], x_{6}:=[1,1,0,1]$, the Cox ring of $X$ is $\mathbb{K}\left[T_{1}, \ldots, T_{15}\right] / I$, where $I$ is generated by

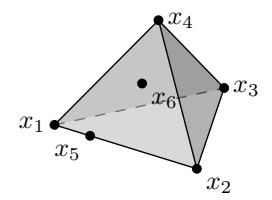

$$
\begin{array}{lr}
T_{1} T_{11}+T_{7} T_{14}+2 T_{8} T_{15}, & T_{2} T_{10}+T_{7} T_{14}+T_{8} T_{15}, \\
T_{4} T_{11} T_{14}-T_{2} T_{13}-T_{5} T_{15}, T_{4} T_{10} T_{14}-T_{1} T_{13}-T_{6} T_{15}, \\
T_{4} T_{10} T_{11}+T_{7} T_{13}-T_{9} T_{15}, & T_{6} T_{11}-2 T_{8} T_{13}-T_{9} T_{14}, \\
T_{5} T_{10}-T_{8} T_{13}-T_{9} T_{14}, & 2 T_{4} T_{8} T_{10}+T_{6} T_{7}+T_{1} T_{9}, \\
T_{4} T_{8} T_{11}+T_{5} T_{7}+T_{2} T_{9}, & T_{4} T_{8} T_{14}+T_{1} T_{5}-T_{2} T_{6}
\end{array}
$$


with the $\mathbb{Z}^{7}$-grading given by the degree matrix

$$
\left[\begin{array}{rrrrrrrrrrrrrrr}
1 & 1 & 1 & 1 & 1 & 1 & 1 & 1 & 1 & 0 & 0 & 0 & 0 & 0 & 0 \\
0 & -1 & -1 & -1 & -1 & 0 & 0 & 0 & 0 & 1 & 0 & 0 & 0 & 0 & 0 \\
-1 & 0 & -1 & -1 & 0 & -1 & 0 & 0 & 0 & 0 & 1 & 0 & 0 & 0 & 0 \\
0 & 0 & 1 & 0 & 0 & 0 & 0 & 0 & 0 & 0 & 0 & 1 & 0 & 0 & 0 \\
-1 & -1 & -1 & 0 & 0 & 0 & -1 & -1 & 0 & 0 & 0 & 0 & 1 & 0 & 0 \\
1 & 1 & 0 & 0 & 1 & 1 & 0 & 1 & 0 & 0 & 0 & 0 & 0 & 1 & 0 \\
1 & 1 & 0 & 1 & 0 & 0 & 1 & 0 & 0 & 0 & 0 & 0 & 0 & 0 & 1
\end{array}\right]
$$

(iii) For $x_{5}:=[1,0,0,1], x_{6}:=[0,1,0,1]$, the Cox ring of $X$ is $\mathbb{K}\left[T_{1}, \ldots, T_{13}\right] / I$, where $I$ is generated by

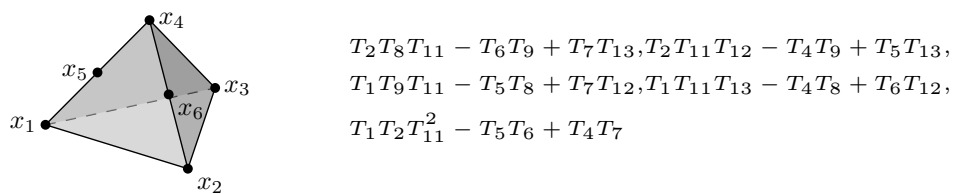

with the $\mathbb{Z}^{7}$-grading given by the degree matrix

$$
\left[\begin{array}{rrrrrrrrrrrrr}
1 & 1 & 1 & 1 & 1 & 1 & 1 & 0 & 0 & 0 & 0 & 0 & 0 \\
0 & -1 & -1 & -1 & -1 & 0 & 0 & 1 & 0 & 0 & 0 & 0 & 0 \\
-1 & 0 & -1 & -1 & 0 & -1 & 0 & 0 & 1 & 0 & 0 & 0 & 0 \\
0 & 0 & 1 & 0 & 0 & 0 & 0 & 0 & 0 & 1 & 0 & 0 & 0 \\
-1 & -1 & -1 & 0 & 0 & 0 & 0 & 0 & 0 & 0 & 1 & 0 & 0 \\
1 & 0 & 0 & 1 & 1 & 0 & 0 & 0 & 0 & 0 & 0 & 1 & 0 \\
0 & 1 & 0 & 1 & 0 & 1 & 0 & 0 & 0 & 0 & 0 & 0 & 1
\end{array}\right]
$$

(iv) For $x_{5}:=[1,0,0,1], x_{6}:=[0,1,1,0]$, the Cox ring of $X$ is $\mathbb{K}\left[T_{1}, \ldots, T_{12}\right] / I$, where $I$ is generated by

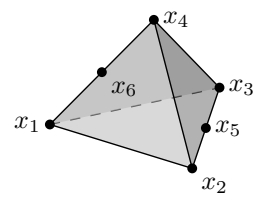

$$
\begin{aligned}
& T_{3} T_{8}-T_{5} T_{12}-T_{2} T_{9}, \\
& T_{4} T_{7}-T_{6} T_{11}-T_{1} T_{10}
\end{aligned}
$$

with the $\mathbb{Z}^{7}$-grading given by the degree matrix

$$
\left[\begin{array}{rrrrrrrrrrrr}
1 & 1 & 1 & 1 & 1 & 1 & 0 & 0 & 0 & 0 & 0 & 0 \\
0 & -1 & -1 & -1 & -1 & 0 & 1 & 0 & 0 & 0 & 0 & 0 \\
0 & 1 & 0 & 0 & 1 & 0 & 0 & 1 & 0 & 0 & 0 & 0 \\
0 & 0 & 1 & 0 & 1 & 0 & 0 & 0 & 1 & 0 & 0 & 0 \\
-1 & -1 & -1 & 0 & -1 & 0 & 0 & 0 & 0 & 1 & 0 & 0 \\
0 & -1 & -1 & 0 & -1 & -1 & 0 & 0 & 0 & 0 & 1 & 0 \\
0 & 1 & 1 & 0 & 0 & 0 & 0 & 0 & 0 & 0 & 0 & 1
\end{array}\right] .
$$

(v) For $x_{5}:=[2,1,0,0], x_{6}:=[1,2,0,0]$, the Cox ring of $X$ is $\mathbb{K}\left[T_{1}, \ldots, T_{12}\right] / I$, where $I$ is generated by

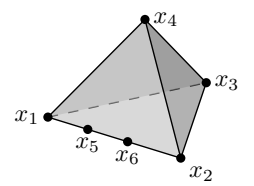

$3 T_{2} T_{7}+2 T_{5} T_{11}+T_{6} T_{12}$,

$3 T_{1} T_{8}+T_{5} T_{11}+2 T_{6} T_{12}$

with the $\mathbb{Z}^{7}$-grading given by the degree matrix

$$
\left[\begin{array}{rrrrrrrrrrrr}
1 & 1 & 1 & 1 & 1 & 1 & 0 & 0 & 0 & 0 & 0 & 0 \\
0 & -1 & -1 & -1 & 0 & 0 & 1 & 0 & 0 & 0 & 0 & 0 \\
-1 & 0 & -1 & -1 & 0 & 0 & 0 & 1 & 0 & 0 & 0 & 0 \\
0 & 0 & 1 & 0 & 0 & 0 & 0 & 0 & 1 & 0 & 0 & 0 \\
0 & 0 & 0 & 1 & 0 & 0 & 0 & 0 & 0 & 1 & 0 & 0 \\
0 & 0 & -1 & -1 & -1 & 0 & 0 & 0 & 0 & 0 & 1 & 0 \\
1 & 1 & 0 & 0 & 1 & 0 & 0 & 0 & 0 & 0 & 0 & 1
\end{array}\right] .
$$

Let $X$ be the blow up of $\mathbb{P}_{3}$ at six non-coplanar points $x_{1}, \ldots, x_{6}$. Denote by $H$ the total transform of a plane of $\mathbb{P}_{3}$ and by $E_{i}$ the exceptional divisor over $x_{i}$. Denote by $E_{I}:=H-\sum_{i \in I} E_{i}$ and by $Q_{i}=2 H-2 E_{i}-\sum_{k \neq i} E_{k}$, the last being the strict transform of the quadric cone with vertex in $x_{i}$ and through the remaining five $x_{j}$. We consider five possibilities for $X$ according to the collinear subsets of $\left\{x_{1}, \ldots, x_{6}\right\}$, modulo permutations of the indices. The meaning of the divisor $\Delta$ in the second column of the table will be explained in the proof of Theorem 7.6 . 


\begin{tabular}{ll}
\hline & $\frac{1}{2}\left(Q_{4}+Q_{5}+Q_{6}+E_{123}\right)$ \\
\hline$\left\{x_{1}, x_{2}, x_{5}\right\}$ & $\frac{5}{8}\left(E_{1235}+E_{1256}+E_{134}+E_{246}+E_{456}\right)$ \\
\hline$\left\{x_{1}, x_{2}, x_{5}\right\},\left\{x_{1}, x_{3}, x_{6}\right\}$ & $\frac{2}{3} E_{12356}+\frac{1}{2}\left(E_{1245}+E_{1346}+E_{234}\right)$ \\
\hline$\left\{x_{1}, x_{2}, x_{5}\right\},\left\{x_{3}, x_{4}, x_{6}\right\}$ & $\frac{1}{3}\left(E_{1235}+E_{1346}+E_{2346}\right)+\frac{1}{2}\left(E_{1245}+E_{1256}+E_{3456}\right)$ \\
\hline$\left\{x_{1}, x_{2}, x_{5}, x_{6}\right\}$ & $\frac{3}{4}\left(E_{12356}+E_{12456}\right)+\frac{1}{3}\left(E_{234}+E_{346}\right)+\frac{1}{4} E_{345}$ \\
\hline
\end{tabular}

Lemma 7.7. Let $X$ be a smooth projective variety, let $D$ be an effective divisor of $X$ and let $C$ be an irreducible and reduced curve of $X$ such that $C \cdot D<0$. Then $C$ is contained in the stable base locus of $|D|$.

Proof. The statement follows from the observation that if $E$ is a prime divisor not containing $C$ then $C \cdot E \geq 0$.

Proposition 7.8. Let $X$ be the blow up of $\mathbb{P}_{3}$ at six non-coplanar points $x_{1}, \ldots, x_{6}$. Denote by $e_{I}$ with $I \subset\{1, \ldots, 6\}$ the class of the strict transform of the line through the points $\left\{x_{i} ; i \in I\right\}$, if $|I| \geq 2$, or the class of a line in the exceptional divisor $E_{i}$ over $x_{i}$, if $I=\{i\}$. Then the Mori cone of $X$ is

$\mathrm{NE}(X)=\left\langle e_{I} ; I \subset\{1, \ldots, 6\},|I|=1\right.$ or the set $\left\{x_{i} ; i \in I\right\}$ is contained in a line $\rangle$.

Proof. We consider five cases for $X$ according to the collinear subsets of $\left\{x_{1}, \ldots, x_{6}\right\}$. In each case we denote by $\mathcal{E}$ be the cone spanned by the classes of the $e_{I}$ defined in the statement. Recall that, via the intersection form between divisors and curves, the nef cone $\operatorname{Nef}(X)$ is dual to the closure of the Mori cone $\operatorname{NE}(X)$ [35, Proposition 1.4.28]. Hence $\mathcal{E} \subset \operatorname{NE}(X)$ gives $\mathcal{E}^{\vee} \supset \operatorname{NE}(X)^{\vee}=\operatorname{Nef}(X)$. Thus, it is enough to show that each extremal ray of $\mathcal{E}^{\vee}$ is a nef class. A direct calculation shows that the primitive generator $w$ of an extremal ray is the strict transform of one of the following divisors (here, by "points" we mean a subset of $\left\{x_{1}, \ldots, x_{6}\right\}$ ):

- a plane through one simple point,

- a quadric through simple points,

- a cubic with one double point and simple points,

- a quartic with one triple point and simple points.

In each case, $w$ is the class of a divisor $D=\sum_{k \in S} E_{I_{k}}$, where $I_{k} \subset\left\{x_{1}, \ldots, x_{6}\right\}$ is a collinear subset for any $k \in S$, where $S$ is a finite set of indices. Hence the base locus of $|D|$ is contained in the union of the strict transforms of the lines spanned by each subset $\left\{x_{i} ; i \in I_{k}\right\}$. Again, a direct calculation shows that $D \cdot e_{I_{k}} \geq 0$ for any $k \in S$ and thus $D$ is nef by Lemma 7.7

Proof of Theorem 7.6. We prove the first statement. The anticanonical divisor $-K_{X}$ of $X$ is big and movable since $-K_{X} \sim 2 D$, where $D=2 H-E_{1}-\cdots-E_{6}$ is the strict transform of a quadric through the six points. Hence, $X$ is Mori dream if and only if it is $\log$ Fano by [38, Lemma 4.10]. We will prove that $X$ is $\log$ Fano by showing that

$$
-K_{X} \sim A+\Delta
$$

where both $A$ and $\Delta$ are $\mathbb{Q}$-divisors, $A$ is nef and big, $\Delta$ is effective, its support is simple normal crossing and $\lfloor\Delta\rfloor=0$. In the above table we provide $\Delta$ in each case. The ampleness of $A$ is a direct consequence of our description of the Mori cone of $X$ given in Proposition 7.8. The second part of the theorem is an application of Algorithm 7.1 . 


\section{REFERENCES}

[1] V.A. Alekseev, V.V Nikulin: Classification of log del Pezzo surfaces of index $\leq 2$. Memoirs of the Mathematical Society of Japan, vol. 15 (2006), Preprint Version arXiv:math.AG/0406536

[2] M. Artebani, A. Garbagnati, A. Laface: Cox rings of extremal rational elliptic surfaces. To appear in Transactions of the AMS. Preprint, arXiv:1302.4361.

[3] I.V. Arzhantsev: On the factoriality of Cox rings. Mat. Zametki 85 (2009), 5, 643-651.

[4] I. Arzhantsev, U. Derenthal, J. Hausen, A. Laface: Cox rings. Cambridge Sudies in Advanced Mathematics 144, Cambridge University Press, Cambridge, 2014. See also arXiv:1003.4229

[5] H. Bäker, J. Hausen, S. Keicher: Chow quotients of torus actions. Preprint, arXiv:1203.3759

[6] L.M. Batten, A. Beutelspacher: The Theory of Finite Linear Spaces. Cambridge University Press, Cambridge, 1993.

[7] V. Batyrev, O. Popov: The Cox ring of a del Pezzo surface Arithmetic of higher-dimensional algebraic varieties (Palo Alto, CA, 2002), 85-103, Progr. Math., 226, Birkhäuser Boston, Boston, MA, 2004.

[8] B. Bechtold: Factorially graded rings and Cox rings. J. Algebra 369 (2012), 351-359.

[9] F. Berchtold, J. Hausen: Homogeneous coordinates for algebraic varieties. J. Algebra 266 (2003), 636-670.

[10] C. Bertone: Modular absolute decomposition of equidimensional polynomial ideals. Preprint, arXiv:1012.5210

[11] W. Bosma, J. Cannon, C. Playoust: The Magma algebra system. I. The user language. J. Symbolic Comput. 24 (1997), no. 3-4, 235-265.

[12] R. de la Bretèche, T.D. Browning, U. Derenthal: On Manin's conjecture for a certain singular cubic surface Ann. Sci. École Norm. Sup. (4) 40 (2007), no. 1, 1-50.

[13] A.-M. Castravet, J. Tevelev: Hilbert's 14th problem and Cox rings. Compos. Math. 142 (2006), no. $6,1479-1498$.

[14] G. Chéze, G. Lecerf: Lifting and recombination techniques for absolute factorization. J. Complexity 23 (2007), no. 3, 380-420.

[15] J.-L. Colliot-Thélène, J.-J. Sansuc: Torseurs sous des groupes de type multiplicatif; applications à l'étude des points rationnels de certaines variétés algébriques C. R. Acad. Sci. Paris Sér. A-B 282 (1976), no. 18, Aii, A1113-A1116.

[16] J.-L. Colliot-Thélène, J.-J. Sansuc: La descente sur une variété rationnelle définie sur un corps de nombres C. R. Acad. Sci. Paris Sér. A-B 284 (1977), no. 19, A1215-A1218.

[17] D.A. Cox: The homogeneous coordinate ring of a toric variety. J. Algebraic Geom. 4 (1995), no. $1,17-50$

[18] W. Decker, G.-M. Greuel, G. Pfister, H. Schönemann: Singular 3-1-6 - A computer algebra system for polynomial computations. 2012. Available at http://www.singular.uni-kl.de

[19] U. Derenthal: Singular Del Pezzo surfaces whose universal torsors are hypersurfaces. Proc. Lond. Math. Soc. (3) 108 (2014), no. 3, 638-681.

[20] U. Derenthal: Geometry of universal torsors. PhD thesis. Universität Göttingen, 2006.

[21] A. Gibney, D. Maclagan: Equations for Chow and Hilbert quotients. Algebra Number Theory 4 (2010), no. $7,855-885$.

[22] D. Grayson, M. Stillman: Macaulay2, a software system for research in algebraic geometry. Available at http://www.math.uiuc.edu/Macaulay2/

[23] G.-M. Greuel, G. Pfister: A Singular Introduction to Commutative Algebra. Second edition. Springer, Berlin, 2008

[24] A. Grothendieck: Eléments de géométrie algébrique. II. Étude globale élémentaire de quelques classes de morphismes. Inst. Hautes Études Sci. Publ. Math. No. 8, 1961

[25] B. Hassett, Y. Tschinkel: Universal torsors and Cox rings. Arithmetic of higher-dimensional algebraic varieties (Palo Alto, CA, 2002), 149-173, Progr. Math., 226, Birkhäuser Boston, Boston, MA, 2004.

[26] J. Hausen: Cox rings and combinatorics II. Mosc. Math. J. 8 (2008), no. 4, 711-757, 847.

[27] J. Hausen: Three Lectures on Cox rings. In: Torsors, étale homotopy and applications to rational points. London Mathematical Society Lecture Note Series 405, 3-60, 2013.

[28] J. Hausen, H. Süß: The Cox ring of an algebraic variety with torus action. Adv. Math. 225 (2010), 977-1012.

[29] E. Huggenberger: Fano Varieties with Torus Action of Complexity One. PhD thesis. Universität Tübingen, 2013, http://nbn-resolving.de/urn:nbn:de:bsz:21-opus-69570.

[30] Y. Hu, S. Keel: Mori dream spaces and GIT. Dedicated to William Fulton on the occasion of his 60th birthday. Michigan Math. J. 48 (2000), 331-348.

[31] A. Jensen: Algorithmic aspects of Gröbner fans and tropical varieties. $\mathrm{PhD}$ thesis, University of Aarhus, 2007.

[32] H. Kraft: Geometrische Methoden in der Invariantentheorie. Aspects of Mathematics, D1. Friedr. Vieweg \& Sohn, Braunschweig, 1984. 
[33] S. Keicher: Algorithms for Mori Dream Spaces. PhD thesis, Universität Tübingen, 2014, http://nbn-resolving.de/urn:nbn:de:bsz:21-dspace-540614.

[34] R. Koelman: The number of moduli of families of curves on toric surfaces. Thesis, Univ. Nijmegen, 1991.

[35] R. Lazarsfeld: Positivity in algebraic geometry I. Ergebnisse der Mathematik und ihrer Grenzgebiete. 3. Folge. A Series of Modern Surveys in Mathematics, 48. Springer-Verlag, Berlin, 2004.

[36] Maple 10. Maplesoft, a division of Waterloo Maple Inc., Waterloo, Ontario. See http://www. maplesoft.com/

[37] K. Matsuki. Introduction to the Mori program. Universitext. Springer-Verlag, New York, 2002.

[38] J. McKernan: Mori dream spaces. Jpn. J. Math. 5 (2010), no. 1, 127-151.

[39] E. Miller, B. Sturmfels: Combinatorial commutative algebra. Graduate Texts in Mathematics, 227. Springer-Verlag, New York, 2005.

[40] S. Okawa: On images of Mori dream spaces. Preprint, arXiv:1104.1326

[41] P. Orlik, P. Wagreich: Algebraic surfaces with $k^{*}$-action, Acta Math. 138 (1977), no. 1-2, 43-81.

[42] A. Sommese, J. Verschelde, C. Wampler: Numerical decomposition of the solution sets of polynomial systems into irreducible components. SIAM J. Numer. Anal. 38 (2001), no. 6, 20222046.

[43] M. Stillman, D. Testa, M. Velasco: Gröbner bases, monomial group actions, and the Cox rings of del Pezzo surfaces. J. Algebra 316 (2007), no. 2, 777-801.

[44] B. Sturmfels: Gröbner bases and convex polytopes. University Lecture Series, 8. American Mathematical Society, Providence, RI, 1996.

[45] B. Sturmfels, M. Velasco: Blow-ups of $\mathbb{P}^{n-3}$ at $n$ points and spinor varieties. J. Commut. Algebra 2 (2010), no. 2, 223-244.

[46] B. Sturmfels, Z. Xu: Sagbi bases of Cox-Nagata rings. J. Eur. Math. Soc. (JEMS) 12 (2010), no. $2,429-459$.

Mathematisches Institut, Universität Tübingen, Auf der Morgenstelle 10, 72076 TüBINGEn, Germany

E-mail address: juergen.hausen@uni-tuebingen.de

Mathematisches Institut, Universität Tübingen, Auf der Morgenstelle 10, 72076 TüBINGEN, GERMANY

E-mail address: keicher@mail.mathematik.uni-tuebingen.de

Departamento de Matemática, Universidad de Concepción, Casilla 160-C, Concepción, Chile

E-mail address: alaface@udec.cl 